\title{
Amazon Nights II: Electric Boogaloo-Neural Adaptations for Communication in Three Species of Weakly Electric Flsh
}

\author{
Kathryne M. Allen \\ West Virginia University, kaallen@mix.wvu.edu
}

Follow this and additional works at: https://researchrepository.wvu.edu/etd

Part of the Neuroscience and Neurobiology Commons

\section{Recommended Citation \\ Allen, Kathryne M., "Amazon Nights II: Electric Boogaloo-Neural Adaptations for Communication in Three Species of Weakly Electric Flsh" (2019). Graduate Theses, Dissertations, and Problem Reports. 3823. https://researchrepository.wvu.edu/etd/3823 \\ This Dissertation is protected by copyright and/or related rights. It has been brought to you by the The Research Repository @ WVU with permission from the rights-holder(s). You are free to use this Dissertation in any way that is permitted by the copyright and related rights legislation that applies to your use. For other uses you must obtain permission from the rights-holder(s) directly, unless additional rights are indicated by a Creative Commons license in the record and/ or on the work itself. This Dissertation has been accepted for inclusion in WVU Graduate Theses, Dissertations, and Problem Reports collection by an authorized administrator of The Research Repository @ WVU. For more information, please contact researchrepository@mail.wvu.edu.}




\title{
Amazon Nights II: Electric Boogaloo \\ Neural adaptations for communication in three species of weakly electric fish
}

Kathryne Allen

\begin{abstract}
A dissertation submitted
to the Eberly College of Arts and Sciences

at West Virginia University

in partial fulfillment of the requirements for the degree of

Doctor of Philosophy

In Biology
\end{abstract}

Gary Marsat, Ph.D., Chair

Andrew Dacks, Ph.D.

Kevin Daly, Ph.D.

Sarah Farris, Ph.D.

G. Troy Smith, Ph.D.

Department of Biology

\section{Morgantown, West Virginia \\ 2018}

Keywords: ethology, neural coding, weakly electric fish, communication, detection, discrimination Copyright 2019 Kathryne Allen 


\begin{abstract}
:
Amazon Nights II: Electric Boogaloo

Neural adaptations for communication in three species of weakly electric fish

Kathryne Allen
\end{abstract}

Sensory systems are the tools by which organisms collect information about the environment. The environments that these systems have evolved to function within shape how they accomplish this task, as each has its own challenges in regards to noise and physical limits of transmission media. In this dissertation, I examine how a unique sensory system, the electrosensory system of three species of weakly electric fishes, is adapted to accomplish the specific task of detecting and accurately encoding conspecific communication. Communication is useful lens for studying how salient signals are filtered from confounding noise and how coding strategies are adapted across species. I specifically show that the physical structure of a signal can dictate how it is encoded by the sensory system, and further demonstrate that different coding strategies are correlated with different perceptual abilities. I then examine how the behavioral use and ethological significance of communication signals also changes which coding strategies are used to encode those signals. Finally, I show that the social context in which signals are received can influence which coding strategies are adopted. 
Dedication

To Mom:

You know I wouldn't be here today without the love, support, and alternating words of reassurance and boots to the ass.

To my sisters:

Thank you all for the wisdom, the understanding ears, and always being there, whether I wanted it or not 
The list of people I have to thank is long, and no matter whom I mention, I am sure this page will fall short of comprehensive.

First, I want to credit all the amazing instructors I have had. Every single one of you have taught me the joy in hard work, curiosity, and to trust in my own abilities. In particular, I need to thank Drs. Ann Fabirkiewicz, Doug Shedd, and Kristin Bliss. You all shaped my undergrad experience and each contributed to my perspectives on both science and life. I especially need to thank Dr. Kurt Siedman. You were exactly the research advisor I needed, if not the one I wanted. I am forever grateful that you chose to herd this cat.

Next, I need to acknowledge my family. The advantage to having a giant, Brady Bunch, mess of a family is that there is always someone who gets it, no matter what "it" is. Micki, Missa, Mere, Jennifer, and Don, thanks for all the wisdom, much of it extremely hard earned. Gene, thank you for being gentle and steadfast. Cathy, thank you for the levity. Dad, thank you for being consistently encouraging. Mom, thank you for literally everything.

Thank you to all of the friends who have brought me joy, perspective, and support through everything. Kayla Burd, Larry Goodwin, Kristyn Lizbinski, Tyler Sizemore, Phil Chapman, and James Roach. I honestly could not have done it without you.

To my labmates in the Marsat Lab, Keshav Ramachandran, Oak Milam, Sree Motipally, Danielle Dillon-Seeger, Daniel Williamson, thanks for all the help and data. You all did great.

Marty, Duke, and Obi, I credit you with my sanity.

To my committee members, Drs. Troy Smith, Andrew Dacks, Kevin Daly, and Sarah Farris, thank you for the help, advice, and knowledge you have given me through the years.

My biggest thanks go to my advisor, mentor, and friend, Dr. Gary Marsat. I don't have the words to fully express everything you have done for me, so let me just say: So long, and thanks for all the fish.

Finally, thank you to the fish, you beautiful little weirdos. 


\section{Table of Contents}

Amazon Nights II: Electric Boogaloo .............................................................................................

Neural adaptations for communication in three species of weakly electric fish................................ i

Abstract:........................................................................................................................... ii

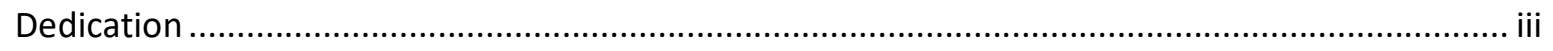

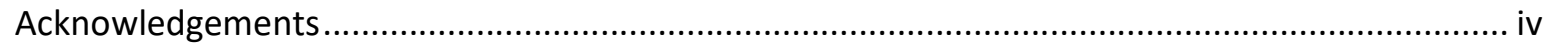

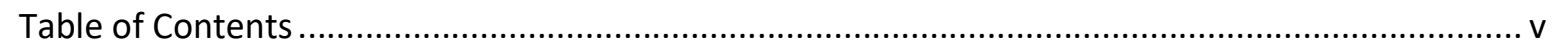

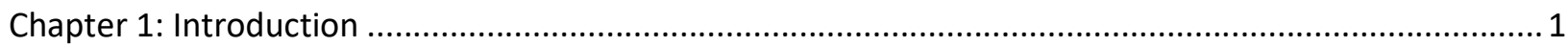

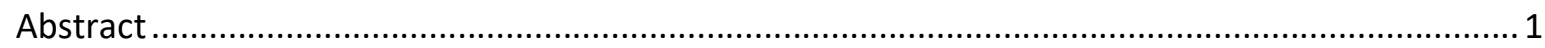

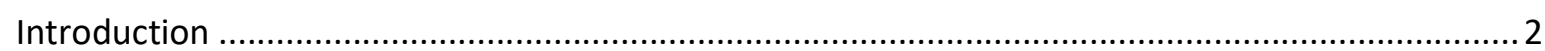

Sender-Receiver Matching .............................................................................................

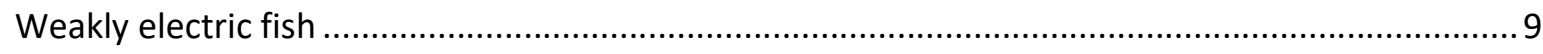

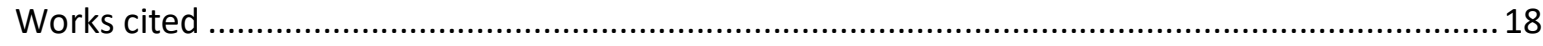

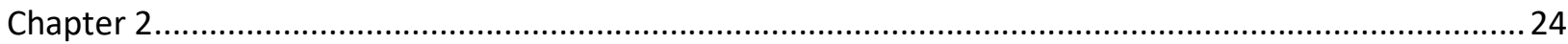

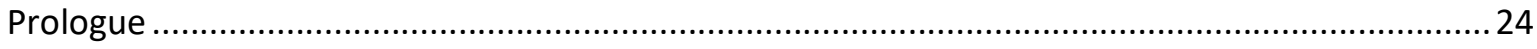

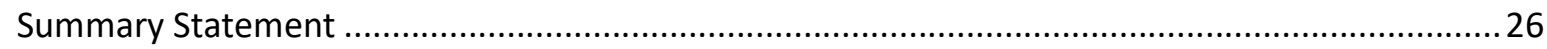

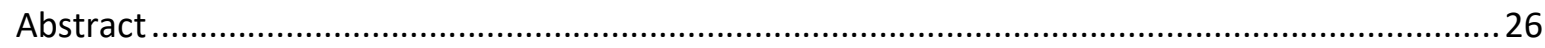

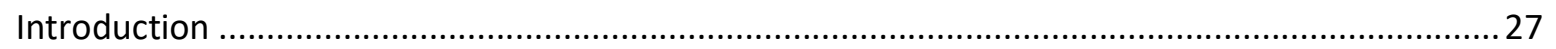

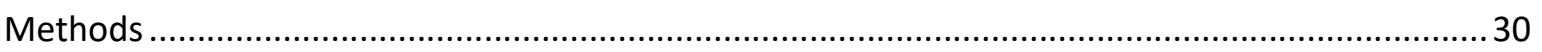

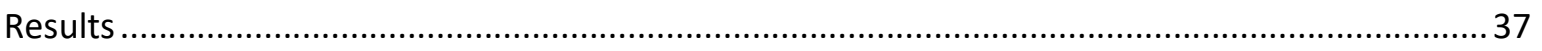

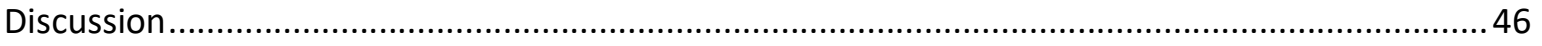

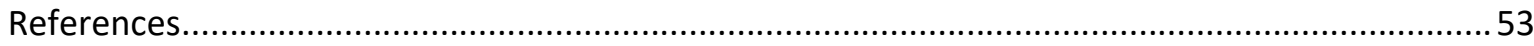

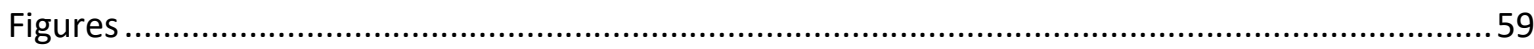

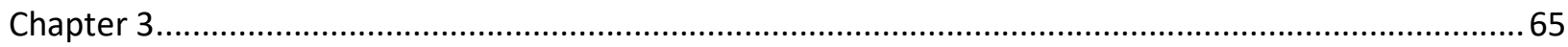

Prologue

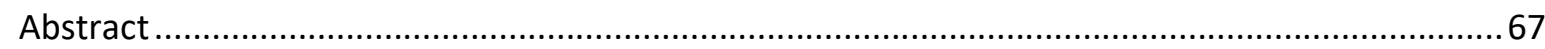

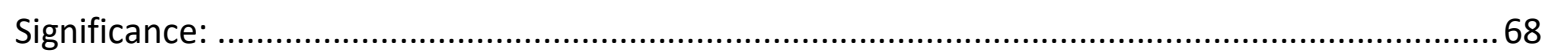

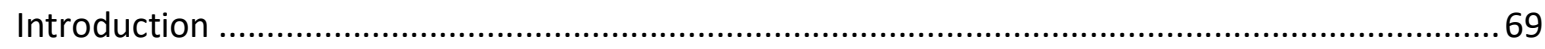

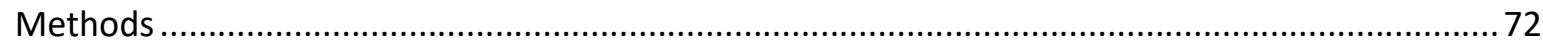

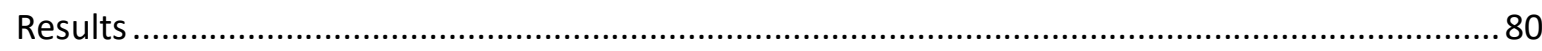

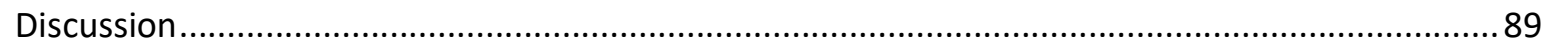

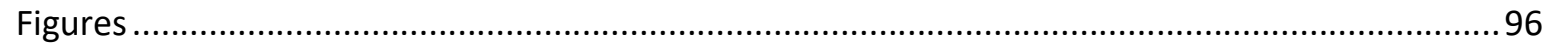

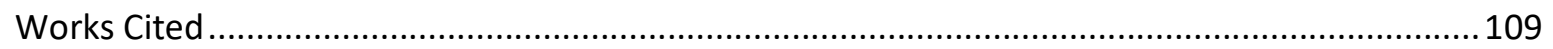




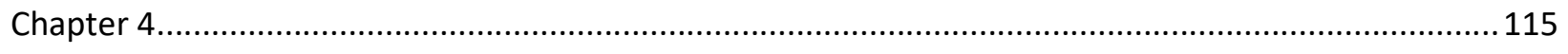

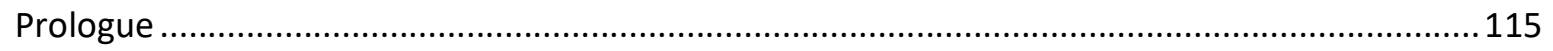

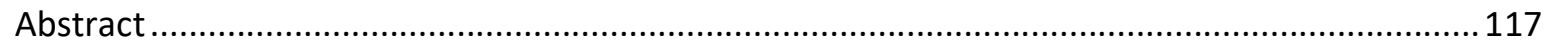

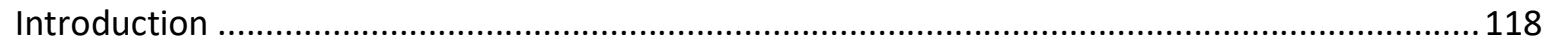

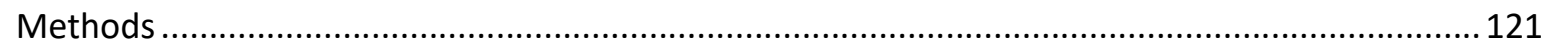

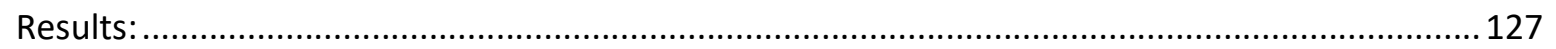

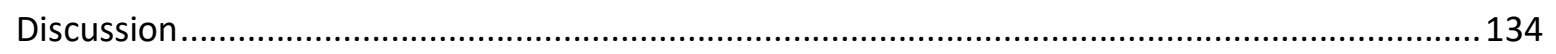

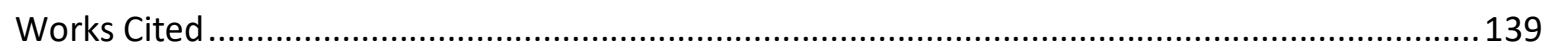

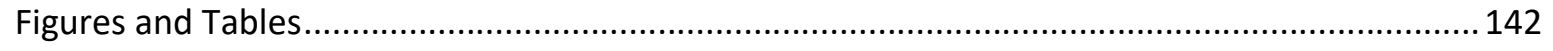

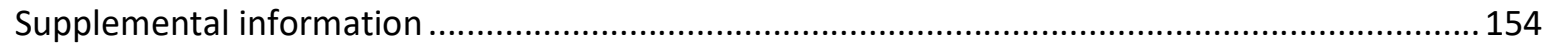

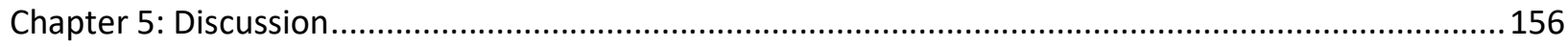

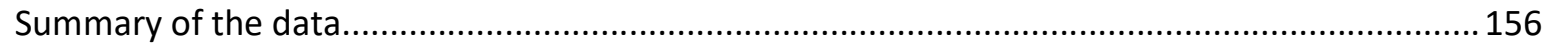

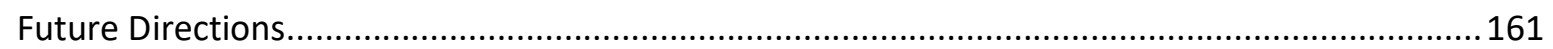

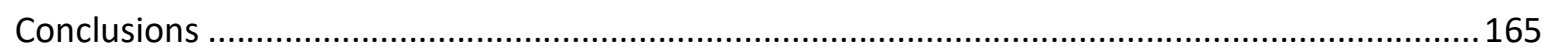

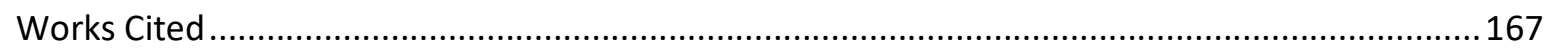




\section{Chapter 1: Introduction}

Abstract

Sensory systems have to extract useful information from environments awash in noise and confounding input. Studying how salient signals are encoded and filtered from these natural backgrounds is a key problem in neuroscience. Communication is a particularly tractable tool for studying this problem, as it is a ubiquitous task that all organisms must accomplish, easily compared across species, and is of significant ethological relevance. In this chapter I describe the current knowledge of what is both known and still unknown about how sensory systems are adapted for the challenges of encoding conspecific signals, particularly in environments complicated by conspecific-generated noise. The second half of this chapter describes why weakly electric fish are particularly suited to investigating how communication can shape the nervous system to accomplish this task. 


\section{Introduction}

Sensory systems must translate physical surroundings into neural representations coded via action potentials. A long-standing goal of neuroscience has been to understand how the intricate internal experience of perception can be tied to this relatively simple physiological mechanism. The study of sensory coding aims to link specific sensory experiences, or perception of sensory signals, to observable and consistent patterns of action potentials.

When studying how sensory systems process input, choosing an appropriate stimulus is key. While entirely artificial stimuli may be easy to administer and control, they risk over simplifying the diverse range of output the system may be capable of producing. Simplistic stimulation regimens are crucial to many important breakthroughs in neural coding, but to understand how brains encode inputs in the real environments, signals that are more realistic must be used. Realistic stimuli provide more nuanced and complete understanding of how sensory signals are processed in the actual situations the organism encounters (Butts et al., 2007; Lewen et al., 2001). Social communication is an ideal realistic stimulus for such a study. Social communication is ubiquitous, but highly diverse, allowing researchers to compare many aspects and stimulus features that sensory systems may be tuned for. It also has large ethological significance, making it easy to tie the stimulus to its natural context and consequence to the organism. Communication also allows for investigation of how long-term changes in context, such as breeding condition and social rank, or short-term changes such as the environmental noise or the identity of the individuals interacting, modulate the reception of signals. Further, the signals and the brains that receive them evolve in conjunction (Endler, 1993), thus communication also presents a unique opportunity to investigate how brains adapt and evolve to extract information in a comparative fashion. Social signaling can be incredibly diverse, even 
among closely related species with similar life histories and selective pressures (Symonds and Elgar, 2008). Due to this diversity, we can often link changes in the sensory system to adaptations for specific sensory needs (Gall et al., 2012).

Based on the strengths outlined above, communication is an ideal stimulus for investigating how adaptations in sensory systems are matched to naturalistic signals. These adaptations can be driven by signal structure, the behaviors involved with signal use, and the context of signal reception. This dissertation will explore how these three components of communication signals shape their reception and neural representation.

\section{Sender-Receiver Matching}

Sender-receiver matching is a widely studied principle in the field of sensory physiology and animal communication. For organisms to exchange information successfully both the motor system producing a signal and the sensory system perceiving the signal should evolve in conjunction so that the sensitive ranges of the sensory system such as frequency tuning match the physical properties of the signal. Sender-receiver matching facilitating communication has been demonstrated repeatedly across diverse groups, including songbirds (Brumm and Slabbekoorn, 2005), anurans (Schul and Bush, 2002), insects (Neuhofer et al., 2008), and stomatopods (Mazel et al., 2004), suggesting that this is a general principle underlying information exchange across species and modalities. Sender-receiver matching in these systems has been investigated in

depth in terms of basic signal features such as carrier frequency (Henry and Lucas, 2008; Konishi, 1970). Other studies have investigated the sensitivity of auditory systems to derived signal features such as pulse duration (Kostarakos and Hedwig, 2012) syllable patterning (Ronacher and Hennig, 2004) or frequency rise (Gall et al., 2012). The ability to recognize 
specific stimulus features efficiently may be particularly important selective markers for identifying conspecifics amongst heterospecific signals in crowded environments.

The above studies are examples of sender-precursors leading to evolutionary change. These examples demonstrate variations in signal structure giving rise to changes in sensory systems. Similarly, receiver-precursor changes can result from adaptations in in the nervous system enabling changes in communication. In Mormyrid fish increased neural plasticity may have allowed for increased diversity in communication structure (Carlson et al., 2011). The development of subdivisions in the midbrain, splitting processing of electrosensory signals into two areas, accompanied by the development of a wider distribution of electroreceptors across the body, gave the resulting phylogenetic branch of fish the flexibility and enhanced processing power to discriminate subtle differences in signal properties. This ability to discriminate conspecific vs. heterospecific signal shape may have led to the explosive diversification seen in this clade (Carlson et al., 2011). Similarly, novel mutations in retinal photopigments have been implicated in speciation and diversification of communication in both butterflies (Sison-Mangus et al., 2010) and cichlids (Seehausen et al., 2008; Terai et al., 2006). Novel changes in the anuran inner ear (Ryan, 2006) also preceded corresponding changes in the frequency of courtship songs. These studies demonstrate that the ability to perceive novel sensory features constitutes a key driver of speciation and promoter of diversity.

While instances of sender-receiver matching are common enough to suggest that it is a universal feature of conspecific communication, there are important instances of sender-receiver mismatching as well. In certain conditions it may be advantageous to generalize for a wide array of sensory features, over narrow specialization for conspecific detection. This may be particularly true for species that rely on the same sensory modality for detection of conspecifics 
as well as prey or predator signals. In the cricket $T$. oceanicus, two peaks in frequency tuning are observed, corresponding to both the carrier frequency of conspecific songs, as well as the ultrasonic frequencies of echolocating bats (Imaizumi and Pollack, 1999; Kostarakos and Hedwig, 2012). Temporal tuning of ultrasound sensitive neurons correspond to pulse rates in bat calls rather than conspecific songs even though the courtship song also has an ultrasonic component that can influence behavior (Marsat and Pollack, 2004; Marsat and Pollack, 2005). Instances of direct mismatching have also been observed in several insects, particularly 17-year cicadas, which seem to be more sensitive to hetero- rather conspecific songs (Römer, 2016).

A key component of determining whether sender-receiver matching is occurring is to examine both the behavior of signal production and physical properties of the signal, but also the physiology of the sensory system in a comparative light. It is unsurprising that diverse input, such as two calls from different species, will produce different responses in the brain of the respective receivers, whether or not there is neural adaptation specifically for the detection of a particular call. However, to confirm that the receiver is matched to the sender the neural responses to stimuli, natural or unnatural, have to be compared to see if there are consistent differences across species. Much of the work in examining sender-receiver matching has been conducted in the sensory periphery. Peripheral tuning and call matching are well characterized, and studies have shown evidence for midbrain adaptations for sender and receiver matching in response to auditory coding of acoustic song (Gerhardt and Schwartz, 2001; Tootoonian et al., 2012; Woolley and Moore, 2011). Within the midbrain, coding tends to become both sparser and more abstract, making it difficult to examine the classic indicators of sender-receiver matching, such as the construction of tuning curves. In more selective brain structures, it may be more useful to investigate responses to more abstract signal features. 


\section{Perceptual tasks and sender-receiver matching}

The extent of sender-receiver matching may not only be influenced by physical properties of the signal, but also how a signal is used and the context in which the signal is received. Breeding condition, social status, group size, and the presence of observers may all affect both the behaviors involved with social communication (Bradbury and Vehrencamp, 2011). Likewise, the context in which signals are received may also affect the perception of communication signals (May et al., 1989). As the natural scenes in which animals are perceiving sensory inputs are so dynamic, it would be disadvantageous to not be able to employ flexible strategies for coding input, even within the limits of sender-receiver matching. An example of this flexibility in coding can be found in Mormyrid fish. In these fish, dynamic modulation of neural filtering properties may be changed by dynamic presynaptic inhibition or excitation. This dynamic tuning may allow these fish to respond both selectively and adaptively to a wide variety of electric signals (Baker et al., 2013).

It is fundamental to consider how much time and energetic resources an animal needs to invest in perceiving a signal. Sometimes an organism merely needs to detect the occurrence of specific signal and assign it to a category without paying attention to the fine variations within a given category. Alternatively, the organism might need to finely evaluate the properties of the signals in order to discriminate meaningful variations (Bradbury and Vehrencamp, 2011; Mitchell et al., 2006; Oglesbee and Kewley-Port, 2009; Richards, 1981). A classic example of the dichotomy between these perceptual strategies can be found in the auditory system of crickets. In crickets, behavioral data show that sound frequency is perceived categorically as either aversive or attractive with a very strict cutoff at $15 \mathrm{kHz}$ between the two (Wyttenbach et 
al., 1996). In contrast, the pulse duration of courtship songs may be ranked as more or less attractive on a gradient (Kostarakos and Hedwig, 2012).

\section{Firing patterns and signal perception}

Two neural coding schemes have repeatedly been linked with the dichotomy of detection and categorical perception vs. discrimination task across a variety of systems. The first coding relies on synchronous high frequency firing, such as bursting, across a population of neurons (Krahe and Gabbiani, 2004; Marsat and Pollack, 2012). The bursts serve a feature extraction mechanism, reliably signaling the occurrence of specific stimulus features while not responding to the bulk of the sensory stream. The second relies on graded responses, particularly gradual increases in tonic firing rate, to support the evaluation and discrimination of stimulus properties such as duration, intensity or modulation (Marsat et al., 2012; Panzeri et al., 2015; Tripathy et al., 2013). Within a sensory system, a given stimulus may evoke either of these two responses depending on the context in which the stimulus is received. In the rodent somatosensory cortex whisker vibration may evoke bursting or tonic firing depending on whether the barrel involved is in an adapted state or not. Correspondingly, in behaving animals asked to perform a whisking discrimination task, adaptation to the stimulus increases performance in discrimination, but also increases the threshold for stimulus detection, echoing the switch from a burst-code to tonic-code (Ollerenshaw et al., 2014). Having the ability to switch between two coding strategies in a context dependent manner increases the ability of the organism to perform well in response to signals in a diverse range of contexts.

Environmental noise and sender-receiver matching

Environmental noise and signal distortion are contextual sources of error in signal 
detection and perception (Brumm and Slabbekoorn, 2005). To deal with environmental effects, an organism must optimize their sensory system and processing strategy. Narrow sensory tuning is advantageous in that only very high-quality stimuli are responded to and false alarms are limited. The cons to narrow tuning are that it can be very limiting in non-ideal conditions. Broader tuning on the other hand is very accommodating and allows for responses over a much wider range of stimuli, but the risk for error or diminishing the impact of any one stimulus becomes problematic. Tree crickets are classic models for studying this problem as ambient temperature can distort the sound of a male's mating call. Therefore, females must either tune their responses to a very narrow range to be responsive to only high-quality males, and risk missing out on those males when temperatures are not favorable, or tune their sensitivities to a broad range of calls at varying temperatures, and risk losing the signal to background noise. In this case, females solve this problem by utilizing a broad tuning strategy at lower stages of sensory perception (Mhatre et al., 2011) that may be modified in in higher order sensory areas (Kostarakos and Hedwig, 2012).

Sociality and noise

When communication signals grow more complex and the context of their usage becomes more complex in conjunction, the strategies used to represent these signals in the brain should also become more nuanced and able to extract information. Sociality and a life history that requires frequent and diverse conspecific interaction drives the evolution of more complicated signal patterns. Many studies have linked increased social complexity with increased signal repertoire and signal complexity (Blumstein \& Armitage, 1997). As the social behavior of a species becomes more complex, communication often needs to become capable of conveying 
more information and gradation, driving the development of new communication channels (Leonhardt, Menzel, Nehring, \& Schmitt, 2016). Much of this prior body of literature has focused on signal production, but very little is known about how sensory processing and signal production evolve together in conjunction with sociality.

A source of noise that is specific to gregarious species is that generated by conspecifics present in a group (Luther and Gentry, 2013). Due to the constraints of sender-receiver matching, the confounding signals in the sensory stream have the very features the sensory system is selective for, consequently, filtering one signal out of many can be challenging. Many species face these problems, particularly those that sing in choruses, such as frogs or songbirds (Bremond, 1978; Wiley, 1994; Pfennig, 2000; Gerhardt \& Huber, 2002; Wollerman \& Wiley, 2002b; Brumm \& Slabbekoorn, 2005; Luther, 2009; Luther \& Wiley, 2009) or those that live in large colonies, such as seabirds (Aubin and Jouventin, 2002). Adaptations to signal structure that facilitate communication in conspecific noise have been studied in a number of systems (Brumm and Slabbekoorn, 2005) as well as the effects of social noise on detection and discrimination levels (Lohr et al., 2003; Wollerman and Wiley, 2002) have been previously studied. However, how the coding schemes that permit the recognition of important signals in conspecific noise compare to those employed by solitary animals is less well understood

\section{Weakly electric fish}

Weakly electric fish are a robust model for studying the questions outlined above. In addition to being a classical neuroethological model, with an extensive history and body of literature, the diversity in communication strategies employed by these fish are particularly conducive for comparative studies. There are two lineages of weakly electric fish, the African 
Mormyridae and the South American Gymnotiformes. Both are valuable models for the study of communication production and reception, but this review will focus on the South American Gymnotiformes, particularly the apteronotid family. This group of fish is particularly favorable to the study of sender and receiver matching for several reasons, outlined below, and will be the focus of my dissertation.

\section{Apteronotid Fish}

Apteronotid electric fish are a branch of the South American gymnotiform order of electric fish. The apteronotid family is diverse, comprised of up to 61 species of fish (Albert and Crampton, 2005), and widespread, inhabiting Amazonian river basins, spanning from Panama to Argentina (Mago-Leccia, 1994). Of these species, many of them are sympatric (Albert and Crampton, 2005; Kramer et al., 2013). This sympatry is a key strength for comparative studies of communication, as it suggests that while these species share habitat, prey, and predation pressures, a possible driver of sensory diversity is diversity in communication.

This family of fishes is distinguished primarily by their neurogenic electric organ (EO), as opposed to the myogenic organ that is typical of all other electric fishes (Kirschbaum, 1983). The fish produce weak electrical fields $(\sim 1 \mathrm{mV} / \mathrm{cm})$ via the EO located along the caudal region (Bennett, 1971) and then detect changes in the amplitude of that field via electroreceptors. This organ, derived from modified spinal motor axons gives them the ability to consistently produce extremely high frequency wave-type electric organ discharges (EOD), up to $2200 \mathrm{~Hz}$ in some species (Albert and Crampton, 2005). This quasi-sinusoidal wave EOD is used by these fish for navigation, prey detection, and communication. 
Much is known about communication behavior in weakly electric fish. As discussed above, the primary medium for conspecific communication in this group is the EOD. The EOD signal of apteronotids is highly regular at a fixed frequency. The EOD waveform and frequency are diverse among species, and waveform in particular may be useful for conspecific recognition in some species (Fugere and Krahe, 2009). Individuals will each have a signature EOD frequency within the species' typical range. This frequency is incredibly stable (Moortgat et al., 2002), but may be changed, both voluntarily and involuntarily, across timescales ranging from milliseconds to minutes (Zakon et al., 2002). Changes in ambient temperature (Dunlap et al., 2000), body mass, and in some species, sex and breeding condition (Smith, 2013) will all produce long-term effects on EOD frequency, and the stable EOD frequency may serve as a useful guide for conspecifics to assess each other. Of the short-term EOD modulations, the most famous example is the Jamming Avoidance Response (JAR). This is a reflex-like adjustment of EOD frequency that occurs when two fish with similar frequencies interact. This type of interaction risks mutual jamming of the low-frequency bandwidth, now occupied by the lowfrequency amplitude modulations (AM) cause by interacting EODs, thus impairing prey capture that relies on signals in the same bandwidth. To prevent this, each fish will adjust its EOD frequency to create a larger difference between the two (Watanabe and Takeda, 1963).

While the mechanisms underlying the encoding of EOD frequency and the production of the JAR are well known, less is understood about the perception of the voluntary, transitory EOD changes used for communication in these fishes. Short increases in EOD frequencies are the most conspicuous form of communication in these fish. They generally can be classified in two categories, Abrupt Frequency Rises (AFR) and chirps. AFRs are characterized by quick increases in EOD frequency of tens of hertz, with relatively long (between 100ms and several 
seconds) returns to baseline frequency. The behavioral role of these frequency changes seems to be tied to active aggressive encounters between males (Hupé and Lewis, 2008; Triefenbach and Zakon, 2008). While AFRs are an interesting behavior, the circumstances of their production are less well understood and thus will not be the focus of this review. The second category, chirps, are named for their "chirp-like" auditory quality when transduced. Chirps are extremely brief $(10-800 \mathrm{~ms})$ marked increases in EOD frequency that can be up to $500 \mathrm{~Hz}$ above the fish's baseline frequency (Zakon et al., 2002). The frequency content, shape, and duration of chirps varies widely between species (Turner et al., 2007) as does the behavioral context of their use (Dunlap and Larkins-Ford, 2003a; Hagedorn and Heiligenberg, 1985). The diversity in properties and usage of these signals suggests that they contain enough information to provide important and specific messages to each species about aggression, courtship and social integration.

Apteronotid chirping behavior is most well-studied in Apteronotus leptorhynchus, a wide spread native of northern countries of South America (De Santana and Fernandes, 2012). In this species, two distinct categories of chirps are produced for different behavioral exchanges. Big, or Type I chirps are long (30-100ms) salient $(300-500 \mathrm{~Hz}$ frequency increases) chirps (Zupanc and Maler, 1993) that are most often produced by males in the context of mating (Hagedorn and Heiligenberg, 1985). Small, or Type II, chirps are much shorter (10-15ms) (Zupanc and Maler, 1993) and produced most frequently by males in agonistic encounters (Hupé and Lewis, 2008) although they have also recently been shown to play a role in courtship (Henninger et al., 2018). Courtship and chirping behavior are heavily influenced by hormonal state. Circulating levels of 11-ketotestosterone is correlated with chirp production in male fish (Dunlap, 2002) and animals of both sexes being treated with testosterone produce more chirps (Dulka et al., 1995; Dunlap, 2002). Apteronotids undergo regular hormonal and developmental fluctuations as they enter and 
exit breeding season (Kirschbaum and Schugardt, 2002) and the hormonal influence of chirping behavior suggests the significant role this exchange of information plays in mating. While we do not know definitively if $A$. leptorhynchus females discriminate among chirp variations, it is likely that courtship success relies on the quality and the detailed structure of the communication signal.

Other apteronotid species produce chirps via the same mechanisms that $A$. leptorhynchus do, but the properties and behaviors of chirping can be quite different. For example many species do not exhibit the same sexual dimorphism in chirp rates that are apparent in A. leptorhynchus (Zakon and Dunlap, 1999). Likewise, chirp rate and chirp use varies dramatically between species (Allen and Marsat, 2019; Kolodziejski et al., 2007). Additionally, the properties of chirps are highly diverse (Dunlap and Larkins-Ford, 2003b; Turner et al., 2007). The diversity in chirp related behaviors and chirp properties help make this group of fish an ideal system for the comparison of communication processing within the brain. As the behavior and structure of chirps vary, we can predict that the electrosensory system should exhibit specific adaptations for these varied communication patterns.

\section{Electroreception}

All weakly electric fish possess the ability to detect weak electric fields created or distorted by objects, prey items, and other fish in their environment. They do this through both the active generation of the EOD as well as passive electroreception. Passive electroreception of low frequency electric fields, such as those created by muscle contractions, is conducted via ampullary electroreceptors (Bullock, 1982). This mode of electroreception is not exclusive to weakly electric fish and is seen in elasmobranchs (Kalmijn, 1966), siluriforms (Dijkgraaf, 1968) and even some mammals (Czech-Damal et al., 2011; Scheich et al., 1986). Active 
electroreception, however, is unique to electric fishes. The fish produce weak electrical fields and then detect changes in the amplitude of that field via tuberous electroreceptors. The change in electric amplitude caused by an object in the fish's vicinity will vary depending on the object's conductivity (increasing for conductive objects, decreasing for resistive objects) and size, allowing the fish to discriminate between various objects and organic materials in the environment. The tuberous electroreceptors are sensitive to much higher frequency changes in electric amplitude than the ampullary receptors (Shumway, 1989). Two types of tuberous receptors respond to different components of the electrical signal. Time coding receptors, called T-units, phase lock to the EOD cycle, firing at each peak of the EOD. Probability coders, or Punits, fire when the amplitude of the electric field increases, and are inhibited by decreases in the field (Bell et al., 1997).

Both T- and P-units synapse on cells in the Electrosensory Lateral Line Lobe (ELL), the primary electrosensory area in the hindbrain. Within the ELL, there are four distinct topographical representations of the surface of the fish's body. The most medial segment (MS) only receives input from ampullary receptors, which the remaining three receive input from the tuberous receptors. The centromedial segement (CMS) centrolateral segment (CLS) and lateral segment (LS) all offer different degrees of resolution of the electrosensory field (Krahe and Maler, 2014). While the exact behavioral role of each of these maps is not entirely explored, lesion experiments suggest that the LS in particular is crucial for detecting and responding to communication signals, and thus will be the focus of this review (Metzner and Juranek, 1997). The LS is characterized by neurons with large receptive fields, particularly along the flanks of the fish, while the other segments tend towards smaller, more precise receptive fields, with a fovea around the face. The resolution and distribution of LS receptive fields reflects the 
significance of this segment in processing conspecific communication, which is quite spatially diffuse compared to the signals of prey items (Milam et al., 2018).

Within the ELL, multiple t-units form gap junctions with spherical cells that then project to the Torus Semicircularis (TS) of the midbrain. T-units and spherical cells code the timing of each EOD zero crossing, which is necessary for the JAR, but they have no known role in processing of communication signals (Walz et al., 2013). P-units, and the pyramidal cells they synapse on, code the amplitude changes that result from chirps and other communication signals. There are two broad classes of pyramidal neurons (Saunders and Bastian, 1984). ON- (also known as E, or Excited) cells receive direct excitatory input from P-units and increase firing in response to increasing electrical field amplitude. OFF- (I, or Inhibited) cells receive input from P-units indirectly, through inhibitory interneurons, and increase firing rate in response to decreases in the electrical field. The neurons are organized in micro-columns with three $\mathrm{ON}$ and three OFF-cells sub-types positioned across the ELL pyramidal cell layer: superficial, intermediate, and deep subtypes. Deep pyramidal cells serve a separate function as they project to the nucleus praeeminentialis and drive direct and indirect feedback loops. Intermediate and superficial pyramidal cells are less categorically distinct (Bastian, 1986) and project to the TS. Several feedback pathways provide inputs to the massive apical dendrite of superficial and intermediate pyramidal cells. Of the known effects of feedback inputs on pyramidal cell responses, the role of parallel-fiber feedback input in cancelling low-frequency redundant signals is most relevant to the processing of communication signals. The cerebellar-like feedback loop provides a "negative image" of the electroreceptor inputs, allowing the cancelation of lowfrequency redundant signals (Bastian, 1986) such as beats occurring during same-sex encounters. 
This feedback not only suppresses the response to the background beat but has been shown to shape and enhance ELL responses to some chirps (Marsat and Maler, 2012).

While pyramidal neurons in the ELL respond to many electrical features, particularly a wide range of chirps, cells of the TS fall into two categories of selective and broad responders, suggesting that this structure serves as an important site for transitioning from dense to sparse coding (Vonderschen and Chacron, 2011). From the TS there are projections to the optic tectum involved in localization of signals, the preglomerular nucleus that serves as a relay to the forebrain, and the nucleus electrosensorious $(\mathrm{nE})$. The $\mathrm{nE}$ is further selective, contains the sign selective cells that mediate the JAR (Carr et al., 1986) and is presumed to mediate the recognition of communication signals. Beside bottom-up inputs from TS, it receives massive topdown inputs for the forebrain and its outputs controls motor areas generating the electro-motor output (Giassi et al., 2012). The $\mathrm{nE}$ thus serves as a higher-level hub coordinating the sensory to motor loop for electrocommunication.

\section{Context and Chirp reception in the ELL}

The sensory system described in the previous section must process the chirp signal in a variety of contexts. There are multiple ways context can influence signal processing, such as the presence of noise, but an omnipresent factor that influences electrocommunication exchanges arises from the way EODs from multiple fish interact during an encounter. Since EODs differ in frequency from fish to fish, they periodically add or subtract from each other, creating an AM equal to the difference in EOD frequency known as a beat. Chirping further modulates this beat (Hagedorn and Heiligenberg, 1985; Zupanc and Maler, 1993) by changing the beat frequency as the chirp unfolds. In A. leptorhynchus, low frequency beats are typical of agnostic encounters between fish of similar sex and size, and fish respond with frequent small chirps $(<100 \mathrm{~Hz}$ 
increase (Hupé and Lewis, 2008)). Higher frequencies occur between fish of opposite sexes or with large differences in body size and more often elicit big chirps $(>100 \mathrm{~Hz})$, typical of courtship (Hagedorn and Heiligenberg, 1985; Henninger et al., 2018). The amplitude modulation occurring during the chirps is thus determined by the increase in EOD of the sender fish, but also by the difference in EOD frequency between the two fish, signaling the type of encounter.

Chirp coding has been best characterized in A. leptorhynchus. In this species, chirp coding is largely mediated by beat frequency. Low beat frequencies cause synchronous firing in the p-units, which is interrupted by chirps. On the contrary, chirps occurring on high beat frequencies result in an increase in p-unit synchrony (Walz et al., 2014). This beat-dependent switch in coding is reflected in the ELL. Small chirps on low frequency beats cause stereotyped bursting. This encoding strategy, and the structure of the signal itself, means variations in small chirps would be difficult to discriminate (Marsat et al., 2009). Conversely, chirps on high frequency beats produce heterogeneous responses which carry the information necessary to discriminate small variations in chirp structure (Marsat and Maler, 2010).

\section{Conclusion}

The existing literature has revealed two different coding strategies depending on chirp type and context that are best suited for two different tasks: detection or discrimination. These patterns are seen in a number of sensory systems. These coding strategies go beyond simple tuning, as the signal is shaped by the initial stages of the system. I noted in previous sections of this chapter that the sender-receiver matching hypothesis relies mostly on evidence derived from the initial stage of sensory processing and basic aspects of the neural response. I argued that understanding sender-receiver matching in a broader context would require focusing on more abstract aspects of the nervous system (e.g. coding strategies) or more complex and naturalistic 
signals such as context of signal reception. I have also detailed why electrocommunication in apteronotids provides the ideal model system to understand how sensory processing is shaped to accomplish these specific tasks by leveraging the diversity in communication characteristics across apteronotid species. In this dissertation, I aim to extend our understanding of the senderreceiver matching principle by comparing the neural coding strategies for naturalistic signals across several species of apteronotids. Considering that sensory processing must be adapted to (1) structure of the signal, (2) how the signal is used and (3) in what context the signal is received, I shed light on this topic from three different perspectives. I first show that in, one species of fish, coding strategies are optimized based on signal structure to accomplish different perceptual tasks (Chapter 2). I then show that signal structure, but also how the signals are used during social interactions, mirrors differences in neural coding strategies across two species (Chapter 3). Finally, I will show that the electrosensory system can reliably encode chirps even in the context of a social group where multiple fish signals are present, but that species that are more social are less affected by this source of noise. These findings, taken together, provide evidence for sender-receiver matching in higher order neural coding schemes.

\section{Works cited}

Albert, J. S. and Crampton, W. G. R. (2005). Diversity and Phylogeny of Neotropical Electric Fishes (Gymnotiformes). In Electroreception (ed. Bullock, T. H.), Hopkins, C. D.), Popper, A. N.), and Fay, R. R.), pp. 360-409. New York, NY: Springer New York.

Allen, K. M. and Marsat, G. (2019). Neural processing of communication signals: The extent of senderreceiver matching varies across species of Apteronotus. eneuro ENEURO.0392-18.2019.

Aubin, T. and Jouventin, P. (2002). How to vocally identify kin in a crowd: the penguin model. Adv. Study Behav. 31, 243-277.

Baker, C. a, Kohashi, T., Lyons-Warren, A. M., Ma, X. and Carlson, B. a (2013). Multiplexed temporal coding of electric communication signals in mormyrid fishes. J. Exp. Biol. 216, 2365-79.

Bastian, J. (1986). Gain control in the electrosensory system: a role for the descending projections to the 
electrosensory lateral line lobe. J. Comp. Physiol. A 158, 505-515.

Bell, C. C., Caputi, A. and Grant, K. (1997). Physiology and plasticity of morphologically identified cells in the mormyrid electrosensory lobe. J. Neurosci. 17, 6409-6423.

Bennett, M. V. L. (1971). Electric organs. In Fish physiology, pp. 347-491. Elsevier.

Bradbury, J. W. and Vehrencamp, S. L. (2011). Principles of Animal Communication. 2nd ed. Sunderland, Mass.: Sunderland: Sinauer Associates.

Brumm, H. and Slabbekoorn, H. (2005). Acoustic Communication in Noise. Adv. Study Behav.

Bullock, T. H. (1982). Electroreception. Annu. Rev. Neurosci. 5, 121-170.

Butts, D. A., Weng, C., Jin, J., Yeh, C. I., Lesica, N. A., Alonso, J. M. and Stanley, G. B. (2007). Temporal precision in the neural code and the timescales of natural vision. Nature 449, 92-95.

Carlson, B. A., Hasan, S. M., Hollmann, M., Miller, D. B., Harmon, L. J. and Arnegard, M. E. (2011). Brain Evolution Triggers Increased Diversification of Electric Fishes. Science (80-. ). 332, 583-586.

Carr, C., Rose, G. J. and Heiligenberg, W. (1986). A Time-Comparison Circuit in the Electric Fish Midbrain. I. Behavior and Physiology. J. Neurosci. 6, 107-119.

Czech-Damal, N. U., Liebschner, A., Miersch, L., Klauer, G., Hanke, F. D., Marshall, C., Dehnhardt, G. and Hanke, W. (2011). Electroreception in the Guiana dolphin (Sotalia guianensis). Proc. R. Soc. B Biol. Sci. 279, 663-668.

De Santana, C. and Fernandes, C. (2012). A New Species of Sexually Dimorphic Electric Knifefish from the Amazon Basin, Brazil (Gymnotiformes: Apteronotidae).

Dijkgraaf, S. (1968). Electroreception in the catfish, Amiurus nebulosus. Experientia 24, 187-188.

Dulka, J. G., Maler, L. and Ellis, W. (1995). Androgen-induced changes in electrocommunicatory behavior are correlated with changes in substance P-like immunoreactivity in the brain of the electric fish Apteronotus leptorhynchus. J. Neurosci. 15, 1879-90.

Dunlap, K. D. (2002). Hormonal and Body Size Correlates of Electrocommunication Behavior during Dyadic Interactions in a Weakly Electric Fish, Apteronotus leptorhynchus. Horm. Behav. 41, 187194.

Dunlap, K. D. and Larkins-Ford, J. (2003a). Production of aggressive electrocommunication signals to progressively realistic social stimuli in male Apteronotus leptorhynchus. Ethology 109, 243-258.

Dunlap, K. D. and Larkins-Ford, J. (2003b). Diversity in the structure of electrocommunication signals within a genus of electric fish, Apteronotus. J. Comp. Physiol. A 189, 153-161.

Dunlap, K. D., Smith, G. T. and Yekta, A. (2000). Temperature Dependence of Electrocommunication Signals and Their Underlying Neural Rhythms in the Weakly Electric Fish, Apteronotus leptorhynchus. Brain. Behav. Evol. 55, 152-162.

Endler, J. A. (1993). Some general comments on the evolution and design of animal communication systems unintended. Philos. Trans. R. Soc. London. Ser. B Biol. Sci. 340, 215-225.

Fugere, V. and Krahe, R. (2009). Electric signals and species recognition in the wave-type gymnotiform fish Apteronotus leptorhynchus. J. Exp. Biol. 213, 225-236. 
Gall, M. D., Brierley, L. E. and Lucas, J. R. (2012). The sender-receiver matching hypothesis: support from the peripheral coding of acoustic features in songbirds. J. Exp. Biol. 215, 3742-3751.

Gerhardt, H. C. and Schwartz, J. J. (2001). Auditory tuning and frequency preferences. Anuran Commun. Smithson. Inst. Press. Washingt.

Giassi, A. C. C., Duarte, T. T., Ellis, W. and Maler, L. (2012). Organization of the gymnotiform fish pallium in relation to learning and memory: II. Extrinsic connections. J. Comp. Neurol. 520, 3338-3368.

Hagedorn, M. and Heiligenberg, W. (1985). Court and spark: electric signals in the courtship and mating of gymnotoid fish. Anim. Behav. 33, 254-265.

Henninger, J., Krahe, R., Kirschbaum, F., Grewe, J. and Benda, J. (2018). Statistics of natural communication signals observed in the wild identify important yet neglected stimulus regimes in weakly electric fish. J. Neurosci. 0350-18.

Henry, K. S. and Lucas, J. R. (2008). Coevolution of auditory sensitivity and temporal resolution with acoustic signal space in three songbirds. Anim. Behav. 76, 1659-1671.

Hupé, G. J. and Lewis, J. E. (2008). Electrocommunication signals in free swimming brown ghost knifefish, Apteronotus leptorhynchus. J. Exp. Biol. 211, 1657-1667.

Imaizumi, K. and Pollack, G. S. (1999). Neural coding of sound frequency by cricket auditory receptors. J. Neurosci. 19, 1508-1516.

Kalmijn, A. J. (1966). Electro-perception in sharks and rays. Nature 212, 1232.

Kirschbaum, F. (1983). Myogenic electric organ precedes the neurogenic organ in apteronotid fish. Naturwissenschaften 70, 205-207.

Kirschbaum, F. and Schugardt, C. (2002). Reproductive strategies and developmental aspects in mormyrid and gymnotiform fishes. J. Physiol. 96, 557-566.

Kolodziejski, J., Sanford, S. E. and Smith, G. T. (2007). Stimulus frequency differentially affects chirping in two species of weakly electric fish: implications for the evolution of signal structure and function. J. Exp. Biol. 210, 2501-2509.

Konishi, M. (1970). Comparative neurophysiological studies of hearing and vocalizations in songbirds. $Z$. Vgl. Physiol. 66, 257-272.

Kostarakos, K. and Hedwig, B. (2012). Calling Song Recognition in Female Crickets: Temporal Tuning of Identified Brain Neurons Matches Behavior. J. Neurosci. 32, 9601 LP-9612.

Krahe, R. and Gabbiani, F. (2004). Burst firing in sensory systems. Nat. Rev. Neurosci. 5, 13-23.

Krahe, R. and Maler, L. (2014). Neural maps in the electrosensory system of weakly electric fish. Curr. Opin. Neurobiol. 24, 13-21.

Kramer, B., Kirschbaum, F. and Markl, H. (2013). SPECIES SPECIFICITY OF ELECTRIC ORGAN DISCHARGES IN A SYMPATRIC GROUP OF GYMNOTOID FISH FROM MANAUS (AMAZONAS). In Sensory Physiology of Aquatic Lower Vertebrates, pp. 195-219.

Lewen, G. D., Bialek, W. and Steveninck, R. R. d. R. v. (2001). Neural coding of naturalistic motion stimuli. Netw. Comput. Neural Syst. 12, 317-329. 
Lohr, B., Wright, T. F. and Dooling, R. J. (2003). Detection and discrimination of natural calls in masking noise by birds: Estimating the active space of a signal. Anim. Behav. 65, 763-777.

Luther, D. and Gentry, K. (2013). Sources of background noise and their influence on vertebrate acoustic communication. Behaviour 150, 1045-1068.

Mago-Leccia, F. (1994). Electric fishes of the continental waters of America. Biblioteca de la Academia de Ciencias Fisicas, Matematicas, y Naturals, Caracas. Venezuela 29, 1-206.

Marsat, G. and Maler, L. (2010). Neural heterogeneity and efficient population codes for communication signals. J. Neurophysiol. 104, 2543-55.

Marsat, G. and Maler, L. (2012). Preparing for the unpredictable: adaptive feedback enhances the response to unexpected communication signals. J. Neurophysiol. 107, 1241-6.

Marsat, G. and Pollack, G. S. (2004). Differential temporal coding of rhythmically diverse acoustic signals by a single interneuron. J. Neurophysiol. 92, 939-48.

Marsat, G. and Pollack, G. S. (2005). Effect of the temporal pattern of contralateral inhibition on sound localization cues. J. Neurosci. 25, 6137-6144.

Marsat, G. and Pollack, G. S. (2012). Bursting neurons and ultrasound avoidance in crickets. Front. Neurosci. 6, 1-9.

Marsat, G., Proville, R. D. and Maler, L. (2009). Transient signals trigger synchronous bursts in an identified population of neurons. J. Neurophysiol. 102, 714-23.

Marsat, G., Longtin, A. and Maler, L. (2012). Cellular and circuit properties supporting different sensory coding strategies in electric fish and other systems. Curr. Opin. Neurobiol. 22, 686-692.

May, B., Moody, D. B. and Stebbins, W. C. (1989). Categorical perception of conspecific communication sounds by Japanese macaques, M acacafuscata. J. Acoust. Soc. Am. 85, 837-847.

Mazel, C. H., Cronin, T. W., Caldwell, R. L. and Marshall, N. J. (2004). Fluorescent Enhancement of Signaling in a Mantis Shrimp. Science (80-. ). 303, 51.

Metzner, W. and Juranek, J. (1997). A sensory brain map for each behavior? Proc. Natl. Acad. Sci. 94,.

Mhatre, N., Bhattacharya, M., Robert, D. and Balakrishnan, R. (2011). Matching sender and receiver: poikilothermy and frequency tuning in a tree cricket. J. Exp. Biol. 214, 2569-2578.

Milam, O., Ramachandra, K. L. and Marsat, G. (2018). Behavioral and Neural Aspects of the Spatial Processing of Conspecifics in the Electrosensory System.

Mitchell, B. R., Makagon, M. M., Jaeger, M. M. and Barrett, R. H. (2006). Information content of coyote barks and howls. Bioacoustics 15, 289-314.

Moortgat, K. T., Keller, C. H., Bullock, T. H. and Sejnowski, T. J. (2002). Submicrosecond pacemaker precision is behaviorally modulated: The gymnotiform electromotor pathway. Proc. Natl. Acad. Sci. 95, 4684-4689.

Neuhofer, D., Wohlgemuth, S., Stumpner, A. and Ronacher, B. (2008). Evolutionarily conserved coding properties of auditory neurons across grasshopper species. Proc. R. Soc. B Biol. Sci. 275, 19651974. 
Oglesbee, E. and Kewley-Port, D. (2009). Estimating vowel formant discrimination thresholds using a single-interval classification task. J. Acoust. Soc. Am. 125, 2323-35.

Ollerenshaw, D. R., Zheng, H. J. V. V, Millard, D. C., Wang, Q. and Stanley, G. B. (2014). The Adaptive Trade-Off between Detection and Discrimination in Cortical Representations and Behavior. Neuron 81, 1152-1164.

Panzeri, S., Macke, J. H., Gross, J. and Kayser, C. (2015). Neural population coding: combining insights from microscopic and mass signals. Trends Cogn. Sci. 19, 162-72.

Richards, D. G. (1981). Alerting and Message Components in Songs of Rufous-Sided Towhees. Behaviour 76, 223-249.

Römer, H. (2016). Matched Filters in Insect Audition: Tuning Curves and Beyond BT - The Ecology of Animal Senses: Matched Filters for Economical Sensing. In (ed. von der Emde, G.) and Warrant, E.), pp. 83-109. Cham: Springer International Publishing.

Ronacher, B. and Hennig, R. M. (2004). Neuronal adaptation improves the recognition of temporal patterns in a grasshopper. J. Comp. Physiol. A Neuroethol. Sensory, Neural, Behav. Physiol. 190, 311-319.

Ryan, M. J. (2006). Neuroanatomy influences speciation rates among anurans. Proc. Natl. Acad. Sci. 83, 1379-1382.

Saunders, J. and Bastian, J. (1984). The physiology and morphology of two types of electrosensory neurons in the weakly electric fish Apteronotus leptorhynchus. J. Comp. Physiol. A 154, 199-209.

Scheich, H., Langner, G., Tidemann, C., Coles, R. B. and Guppy, A. (1986). Electroreception and electrolocation in platypus. Nature 319, 401.

Schul, J. and Bush, S. L. (2002). Non-parallel coevolution of sender and receiver in the acoustic communication system of treefrogs. Proc. R. Soc. London. Ser. B Biol. Sci. 269, 1847 LP-1852.

Seehausen, O., Terai, Y., Magalhaes, I. S., Carleton, K. L., Mrosso, H. D. J., Miyagi, R., van der Sluijs, I., Schneider, M. V, Maan, M. E., Tachida, H., et al. (2008). Speciation through sensory drive in cichlid fish. Nature 455, 620-U23.

Shumway, C. A. (1989). Multiple electrosensory maps in the medulla of weakly electric gymnotiform fish. I. Physiological differences. J. Neurosci. 9, 4388-99.

Sison-Mangus, M. P., Bernard, G. D., Briscoe, A. D., Warren, A. D., Bybee, S. M., Reed, R. D., Yuan, F., Chiao, C.-C. and Llorente-Bousquets, J. (2010). Positive selection of a duplicated UV-sensitive visual pigment coincides with wing pigment evolution in Heliconius butterflies . Proc. Natl. Acad. Sci. 107, 3628-3633.

Smith, G. T. (2013). Evolution and hormonal regulation of sex differences in the electrocommunication behavior of ghost knifefishes (Apteronotidae). J. Exp. Biol. 216, 2421 LP-2433.

Symonds, M. R. E. and Elgar, M. A. (2008). The evolution of pheromone diversity. Trends Ecol. Evol. 23, 220-228.

Terai, Y., Seehausen, O., Sasaki, T., Takahashi, K., Mizoiri, S., Sugawara, T., Sato, T., Watanabe, M., Konijnendijk, N., Mrosso, H. D. J., et al. (2006). Divergent Selection on Opsins Drives Incipient Speciation in Lake Victoria Cichlids. PLOS Biol. 4, e433. 
Tootoonian, S., Coen, P., Kawai, R. and Murthy, M. (2012). Neural Representations of Courtship Song in the Drosophila Brain. J. Neurosci. 32, 787 LP-798.

Triefenbach, F. A. and Zakon, H. H. (2008). Changes in signalling during agonistic interactions between male weakly electric knifefish, Apteronotus leptorhynchus. Anim. Behav. 75, 1263-1272.

Tripathy, S. J., Padmanabhan, K., Gerkin, R. C. and Urban, N. N. (2013). Intermediate intrinsic diversity enhances neural population coding. Proc. Natl. Acad. Sci. U. S. A. 110, 8248-8253.

Turner, C. R., Derylo, M., de Santana, C. D., Alves-Gomes, J. a and Smith, G. T. (2007). Phylogenetic comparative analysis of electric communication signals in ghost knifefishes (Gymnotiformes: Apteronotidae). J. Exp. bBology 210, 4104-4122.

Vonderschen, K. and Chacron, M. J. (2011). Sparse and dense coding of natural stimuli by distinct midbrain neuron subpopulations in weakly electric fish. J. Neurophysiol. 106, 3102-18.

Walz, H., Hupe, G. J., Benda, J. and Lewis, J. E. (2013). The neuroethology of electrocommunication: How signal background influences sensory encoding and behaviour in Apteronotus leptorhynchus. J. Physiol. 107, 13-25.

Walz, H., Grewe, J. and Benda, J. (2014). Static frequency tuning accounts for changes in neural synchrony evoked by transient communication signals. J. Neurophysiol. 112, 752-65.

Watanabe, A. and Takeda, K. (1963). The Change of Discharge Frequency by A.C. Stimulus in a Weak Electric Fish. J. Exp. Biol. 40, 57.

Wollerman, L. and Wiley, R. H. (2002). Background noise from a natural chorus alters female discrimination of male calls in a Neotropical frog. Anim. Behav. 63, 15-22.

Woolley, S. M. N. and Moore, J. M. (2011). Coevolution in communication senders and receivers: vocal behavior and auditory processing in multiple songbird species. Ann. N. Y. Acad. Sci. 1225, 155-165.

Wyttenbach, R. A., May, M. L. and Hoy, R. R. (1996). Categorical Perception of Sound Frequency by Crickets. Science (80-. ). 273, 1542-1544.

Zakon, H. H. and Dunlap, K. D. (1999). Sex steroids and communication signals in electric fish: a tale of two species. Brain. Behav. Evol. 54, 61-9.

Zakon, H., Oestreich, J., Tallarovic, S. and Triefenbach, F. (2002). EOD modulations of brown ghost electric fish: JARs, chirps, rises, and dips. J. Physiol. Paris 96, 451-8.

Zupanc, G. K. H. and Maler, L. (1993). Evoked chirping in the weakly electric fish Apteronotus leptorhynchus : a quantitative biophysical analysis. Can. J. Zool. 71, 2301-2310. 


\section{Chapter 2}

Prologue

In A. leptorhynchus it has been shown that small chirps on low frequency beats produce responses that are stereotyped and do not provide enough information to discriminate subtle changes in chirp length or frequency (Marsat et al., 2009). Conversely, big chirps on high frequency beats produce responses that are information rich and allow for efficient chirp discrimination (Marsat and Maler, 2010). What is not known is whether this switch in coding strategy is mediated by the change in frequency or the change in chirp structure. Evidence from electroreceptor recordings suggest that the frequency of the beat is a driving factor in this switch. Low frequency amplitude modulations cause synchronization in firing among p-units, and chirps interrupt that synchrony (Walz et al., 2014). On high frequency beats, electroreceptor firing is desynchronized and of both types chirps increase synchronization (Walz et al., 2014). This suggests that beat frequency, rather than the chirp type mediates the switch between burst and tonic firing.

In this chapter, I use both neurophysiology and a behavioral test to determine both how beat frequency affects coding, and if these two coding strategies, burst vs. tonic firing, do lead to different levels of discrimination between chirps. My data show that in A. leptorhynchus we can correlate these two patterns of firing, burst vs. tonic, with two different perceptual abilities. Chirps coded with burst firing cannot be discriminated, while those coded with tonic firing are discriminable.

My results argue that these neural codes used in different situations are matched to the perceptual tasks performed by the animal. The results thus lead to the prediction that, in this 
system, there is a tradeoff between sensitive detection and accurate discrimination and coding efficiency is maintained by changing coding scheme.

Note: This chapter is taken directly from my publication:

"Task-specific sensory coding strategies are matched to detection and discrimination performance", Kathryne M. Allen, Gary Marsat, Journal of Experimental Biology 2018, 221:jeb170563, Published 27 March 2018.

Gary Marsat designed the experiments and collected and analyzed the behavioral data, Kate Allen collected and analyzed the electrophysiology data. 


\section{Summary Statement}

In this paper, we show that by using two distinct neural coding schemes, weakly electric fish sensory neurons change response patterns to adapt to context-specific perceptual needs.

\section{Abstract}

The acquisition of sensory information is limited by the neural encoding method used, constraining perceptual abilities. The most relevant aspects of stimuli may change as behavioral context changes, making efficient encoding of information more challenging. Sensory systems must balance rapid detection of a stimulus with perception of fine details that enable discrimination between similar stimuli. We show that in a species of weakly electric fish, Apteronotus leptorhynchus, two coding strategies are employed for these separate behavioral tasks. Using communication signals, we demonstrate a strong correlation between neural coding strategies and behavioral performance on a discrimination task. Extracellular recordings of pyramidal cells within the electrosensory lateral line lobe of alert fish show two distinct response patterns, either burst discharges with little variation between different signals of the same category, or a graded, heterogeneous response that contains sufficient information to discriminate between signals with slight variations. When faced with a discrimination-based task, the behavioral performance of the fish closely matches predictions based on coding strategy. Comparisons of these results with neural and behavioral responses observed in other model systems suggest that our study highlights a general principle in the way sensory systems utilize different neural codes. 


\section{Introduction}

Behavioral context can dramatically affect the perception of sensory signals and the most relevant aspects of the signal can vary with these changes in context. Sometimes an organism merely needs to detect a specific signal within a continuous and noisy sensory stream.

Alternatively, the organism might need to finely evaluate the properties of the signals in order to discriminate meaningful variations (Bradbury, 2011; Kröger et al., 2011; Mitchell et al., 2006; Oglesbee and Kewley-Port, 2009; Richards, 1981). Our goal in this study is to demonstrate that these two different sensory needs -detection vs. discrimination- are met with different sensory coding schemes specialized for different contexts.

Neuroscience research has long been concerned with deciphering the relationship between the pattern of activity of sensory neurons and perception. The challenges faced when studying this question can be divided into three: 1-understanding how relevant sensory information is represented in the neural code; 2- probing sensory abilities through behavior; and 3- correlating the two into a cohesive understanding of "perception". The relationship between the sensory codes and sensory abilities of organisms has been explored in many systems (e.g. Arabzadeh et al., 2003; Freedman et al., 2001; Tremblay et al., 1996; von Heimendahl et al, 2007). In the visual and vibrissae systems neural codes change as adaptation to a stimulus sets in. After adaptation, the initial low-threshold detectability of a novel stimulus is traded for increased discriminability between similar stimuli (Fairhall et al., 2001; Moore, 2004) a change reflected in the behavioral performance of the animal (Ollerenshaw et al., 2014).

The two neural coding schemes observed in this system have repeatedly been linked with detection vs. discrimination tasks in various other systems. The first coding strategy relies on synchronous high frequency firing - typically bursting-over a population, for detection of 
important stimulus features (Krahe and Gabbiani, 2004; Marsat and Pollack, 2012). The second relies on graded responses with heterogeneous firing across the population to support the evaluation and discrimination of fine details of the stimulus (Marsat et al. 2012; Panzeri et al., 2015; Tripathy et al., 2013). The relationship between these two strategies and stimulus encoding is firmly established, but we have yet to demonstrate that they indeed are systematically mediating different behavioral tasks. Here we use the weakly electric fish communication system to demonstrate a close association between the stimulus encoding method and the associated behavior. We propose a general principle linking these sensory codes to two different perceptual tasks: detection vs. discrimination.

Weakly electric fish communication signals, chirps, are transient modulations of their ongoing oscillating electric field, or EOD (electric organ discharge). Two fish interacting will perceive each other's field as quasi-sinusoidal modulations of their own EOD -called beats-and chirps as transient disruptions of this regular background. In typical male-female courtship interactions, signals will consist of high frequency beats due to the sexual dimorphism of EOD frequency. Type 1 (big) chirps are produced most often in this context (Hagedorn and Heiligenberg, 1985; Hupé and Lewis, 2008). Low frequency beats are more typical of same-sex encounters such as male-male aggressive interactions. Low frequency beats elicit the production of frequent type 2 (small) chirps (Hupé and Lewis, 2008). Neurophysiological recordings of the primary electrosensory area in the brain, the electrosensory lateral line lobe (ELL), show that small chirps on low frequency beats are encoded by synchronized bursting among the population of pyramidal cells (Marsat et al., 2009), consistent with a feature detection code. Courtship signals, big chirps on high frequency beats, are encoded via graded, heterogeneous firing (Marsat and Maler, 2010). This latter response type, but not the former, can support the efficient 
discrimination of small variations in chirp properties, possibly to evaluate the quality of the courtship signal.

Low frequency beats, small chirps are typically associated with agonistic encounters, whereas high frequency beats and big chirps have been suggested as typical courtship signals. However, these patterns are not set rules. Recent field studies report the use of small chirps paired with high frequency beats during courtship, a behavior not previously reported in lab studies, and therefore not previously examined in detail (Henninger, 2015; Henninger et al., 2017). Given the nature of the signals and the neural responses described in this introduction, this system allows us to ask if the neural code employed is simply a function of the signal or is it dependent on context and consistently matched to the behavioral task in order to enhance either the capacity to discriminate or simply detect the stimulus? 


\section{Methods}

Neurophysiology:

Surgical methods are based on those described in Marsat, Proville, and Maler (2009) and Marsat and Maler (2010). Fish of both sexes were anaesthetized and respirated with a solution of Tricaine-S (Tricaine Methanesulfonate, Western Chemical, Inc.) in water $(.25 \mathrm{~g} / \mathrm{L})$ for the duration of the surgery. After the application of a local anesthetic (Lidocaine $\mathrm{HCl} \%$, Hospira, Inc.) skin and overlying soft tissues were removed from a small area of the skull. A portion of the exposed skull was glued to a fixed post for stability while the portion of the skull overlying the ELL was then removed. Fish were immobilized with a $.1 \mathrm{~mL}$ injection of Tubocurarine Chloride Pentahydrate $(.2 \mathrm{mg} / \mathrm{mL}, \mathrm{TCI})$, switched to anesthetic free water for respiration, and allowed to recover from surgery for approximately 20 minutes in the experimental tank before stimulation and recording. The experimental tank $(40 \times 45 \times 20 \mathrm{~cm})$ contained water matched to the home system $\left(26-27^{\circ} \mathrm{C}, 250-300 \mathrm{uS}\right)$. In vivo recordings of the Lateral Segment (LS) of the ELL were made via metal filled extracellular electrodes (Frank and Becker, 1964) and amplified (A-M Systems, Model 1700), and data recorded (Axon Digidata 1500 and Axoscope software) at a $20 \mathrm{kHz}$ sampling rate. Pyramidal cells of the LS were targeted and identified by location in relation to major surface blood vessels, depth from the surface of the brain, as well as neural response properties (Maler, 1991; Saunders and Bastian, 1984).

\section{Stimulation during neurophysiology}

All stimuli were sampled at $20 \mathrm{kHz}$ and created using MATLAB (Mathworks, Inc.). Pyramidal cells respond to changes in EOD amplitude (Bastian 1981; Saunders and Bastian 1984), so stimulation was provided by a direct modulation of a carrier frequency matching the fish's own rather than by mimicking a second EOD. This method allows for tight control over the signal the fish receives and is commonly used in similar experiments (Bastian and 
Heiligenberg 1980; Benda et al. 2005). Although this stimulation method does not replicate the phase modulation component of natural communication signals, only T-unit electroreceptors encode this stimulus feature. Very few T-unit electroreceptors are found in A. leptorhynchus and they do not provide direct inputs to pyramidal cells (Maler et al., 1981). P-unit receptors (those that encode AM signals) drive pyramidal cells. These electroreceptors respond in an identical manner to AM only or AM + EOD phase stimuli (Benda et al., 2005). The responses of the pyramidal cells to these types of stimuli are not significantly different (unpublished data, see Marsat et al., 2014)

The baseline EOD was recorded via electrodes near the head and tail of the fish. Each EOD cycle triggered a sine wave generator (Rigol DG1022A) to generate one cycle of a sine wave matched to the animal's own. This signal was then multiplied using a custom-built signal multiplier (courtesy of the Fortune Laboratory, New Jersey Institute of Technology) by the AM stimulus to create the desired modulation of the electric field around the fish. It was played through a stimulus isolator (A-M Systems, Model 2200) into the experimental tank via two $30.5 \mathrm{~cm}$ electrodes. The electrodes were placed on either side of the fish close to the tank walls and parallel to the fish's longitudinal axis. This arrangement produces a fairly uniform stimulation of the majority of the skin's surface. The stimulus strength was adjusted to provide $\sim 20 \%$ contrast (the difference between the maximum and baseline EOD amplitude relative to baseline).

Chirp stimuli consisted of a Gaussian shaped frequency modulation of the background beat presented once per second on either a $10 \mathrm{~Hz}$ (low frequency beat; LFB) or $120 \mathrm{~Hz}$ beat (high frequency beat; HFB). The frequency and duration of chirps were chosen to mimic a range of natural signals (Bastian et al. 2001). Small chirps were either $10 \mathrm{~ms}$ long with a $60 \mathrm{~Hz}$ 
increase, or $15 \mathrm{~ms}$ long with a $122 \mathrm{~Hz}$ increase. Big chirps were either $15 \mathrm{~ms}$ long with a $300 \mathrm{~Hz}$ increase, or $45 \mathrm{~ms}$ long with a $900 \mathrm{~Hz}$ increase. Chirp frequency increase was tied to a signal amplitude decrease of $0.08 \%$ for each $\mathrm{Hz}$ of frequency rise, based on natural chirp properties (Zupanc and Maler 1993). All four chirps were played on both the 10 and $120 \mathrm{~Hz}$ beats but responses to big chirps on the $10 \mathrm{~Hz}$ beat were not examined in detail. Small chirps were presented at several different phases of the beat, typically at the peak or trough of the sine wave.

The present study is not intended as an exhaustive characterization of responses to chirps as this information is already available (e.g. Marsat and Maler, 2010; Marsat et al., 2009; Metzen et al., 2016; Vonderschen and Chacron, 2011). Rather, we use a single pair of chirps for each stimulus category and choose the chirps properties of the pair to be as different as possible in both duration and frequency increase given observed natural ranges (Bastian et al., 2001). This makes the discrimination task as easy as possible for the animal while keeping the chirp properties realistic for each category.

\section{Neural data analysis}

Analysis of discrimination (Fig1-3) is based on Marsat and Maler (2010) and modifications to methods originally described by van Rossum (van Rossum, 2001). This method accounts for both the firing rate as well as the temporal pattern of spikes to quantify how similar or dissimilar spiking patterns are. Our analysis is mathematically equivalent to previous approaches (Laubach et al., 2000; Stecker et al., 2005; Vonderschen and Chacron, 2011) but instead of displaying a confusion matrix and calculating the amount of information carried by the population response about stimulus identity we display the ROC curves and quantify an error level. 
Spike trains were binarized and convolved with an $\alpha$ filter, $f(t)=t^{-2.45 / \tau}$, with $\tau$ being the width of the function at half maximum (Machens et al., 2003). A portion of the result $R(t)$ was extracted for analysis, specifically, a window around the timing of the presented chirps $(-15$ to $30 \mathrm{~ms}$ relative to the middle of big chirps and -10 to $30 \mathrm{~ms}$ relative to small chirps). The distance $D_{x y}$ between the two spike trains $x$ and $y$ of length $L$ is defined as:

$D_{x y}=1 / L * \sum_{t=0}^{L}\left[R_{z}(t)-R_{y}(t)\right]^{2}$. Larger distances indicate more dissimilar spike trains. In addition to the response of individual neurons, we looked at population responses by averaging several spike trains using the function $P R(t)=\sum_{i=t}^{n}[R i(t)] / n$. The result $(P R(t))$ represents a population of neurons presented with the same stimulus and mimics a neuron integrating postsynaptic potentials with similar weights (Larson et al., 2009). These population responses were created by randomly pairing multiple individual responses, simulating the response of a diverse population of cells. Up to 200 random combinations of spike trains from all recorded neurons were used for all comparisons. Responses to different chirps (X vs. Y) were compared as well as multiple responses to the same chirp (X vs. X). Distance $\left(D_{X Y}\right.$ or $\left.D_{x x}\right)$ was calculated for all sets of combined responses, $P R_{\mathrm{x}}(t)$ and $P R_{\mathrm{y}}(t)$, creating an array of response distances for each comparison. The probability distributions of the values in these arrays $\left(P\left(D_{X Y}\right)\right.$ or $\left.P\left(D_{x x}\right)\right)$ were used for ideal observer analysis. Receiver operating characteristic curves were generated by varying the threshold distance for discrimination, T. For each threshold value, the probability of non-discrimination $\left(P_{\mathrm{D}}\right)$ is calculated as the sum of $P\left(D_{\mathrm{XY}}>T\right)$, and the probability of false discrimination $\left(P_{\mathrm{F}}\right)$ as the sum of $P\left(D_{\mathrm{XX}}>T\right)$. The error level for each threshold value is $E=$ $1 / 2 P_{\mathrm{F}}+1 / 2\left(1-P_{\mathrm{D}}\right)$. The error in discrimination reported in the figures are the minimum values of $E$. This measure of error rate is closely related to the Kullback-Leibler divergence quantifying how much overlap two distributions have. These measures thus quantify how different two 
distributions are without relying on assumptions of normality as other statistical tests often do. Jackknife resampling (leaving one neuron at a time out of the analysis) was used to calculate the standard errors displayed in Figures 1-3. Sample sizes for these figures are equal to the maximal value of the $\mathrm{x}$-axis plus one.

Even though it is relatively simple, our spike metric distance analysis is based on a principle that can be implemented by a biologically realistic decoder (Larson et al., 2009). More complex decoders that could be implemented by neural circuits have been tested but failed to allow discrimination of small chirp LFB signals (unpublished data, see: Sharpee et al., 2016).

\section{Behavioral paradigm}

The experimental tank measured $30 \mathrm{~cm} \times 30 \mathrm{~cm} \times 10 \mathrm{~cm}$ and contained water matched to the fish's home tank $\left(26-27^{\circ} \mathrm{C}\right.$ and a conductivity of $\left.250-300 \mu \mathrm{S}\right)$. Fish were allowed to acclimate overnight. The experimental tank was shielded from light and illuminated with an infrared light source and filmed with an infrared camera (Logitech C920, with its IR filter removed). Video was captured at 24 frames/s and a spatial resolution of $1280 \times 720$ pixels (the tank covered the width of the frame). Stimuli were played via a pair of submerged electrodes (dipole spacing $=10 \mathrm{~cm}$ ) located in one quadrant of the tank (Fig 4A) at a strength replicating the average fish's electric field. The small size of the behavior tank ensures that no matter the position of the fish in the tank, the stimulus received will be fairly strong and should not affect the reception of the chirp stimulus.

Fish were played one of three chirp stimuli: big chirps on a $120 \mathrm{~Hz}$ beat, small chirps on a $10 \mathrm{~Hz}$ beat, or small chirps on $120 \mathrm{~Hz}$ beat. Chirp properties were as described for the 
neurophysiology experiments. These chirps were played at a rate of twice per second for one minute, followed by a two-minute break with no stimulation. This stimulation pattern was repeated for 90 minutes to habituate the fish to the chirp. After 90 minutes, a chirp of the same category (either small or big) but with a different frequency increase and duration was played on the same beat frequency (Fig 4B). Behavior was assessed throughout the experiment when the stimulus was $\mathrm{ON}$ to be able to quantify the habituation and possible dishabituation of the response. Recordings were acquired from 250 trials. A given fish was tested only once a day and could be tested up to three times total, once with each stimulus.

\section{Behavioral Analysis}

The video files were imported in MATLAB where a custom program was used to analyze the video frame-by-frame. The semi-automatic analysis identified the position of three points on the fish: the tip of the nose, the tip of the tail and the 2D center of mass. These points were determined automatically by the program but visual inspection of the results was required to correct occasional errors (e.g. flipping of the tail and nose). Swimming speed and position could easily be calculated given the frame time stamp and the spatial calibration. The results displayed took into account the position of the nose, but the "center of mass" gave similar results; results using the tip of the tail were not evaluated.

For each 1-minute stimulus bout, the swimming speed was calculated (Fig 4) based on the distance moved between video frames. A probability distribution of swimming speed for each stimulation bout was then calculated. When the fish was swimming actively (relatively high speed), we noticed that the strength of the overall response was not most strongly correlated to the swimming speed, but rather that the time spent swimming actively at relatively high speed was a better indication of the response strength. To quantify swimming speed in a way that 
accounts for these characteristics, we calculated a relative swimming speed ratio. Using the three stimulation bouts preceding the chirp-change (i.e. habituated state) we calculated a median swim speed and used it as a threshold to define two ranges of speed: slow or fast. We defined a speed index as the ratio of the proportion of time where swimming is fast vs. slow. Using the speed threshold determined from the trials preceding the chirp-change allows us to normalize this speed index to 1 for the habituated state. If the fish spends more time swimming at fast speeds, the speed index will be above 1 (e.g. index of 3 if the fish swims fast $75 \%$ of the time vs $25 \%$ at slow speed). Speed indexes are not normally distributed, thus data were transformed with an exponential function to change the distribution into a normal one for statistical testing with a paired t-test in figure 5C.

Quantifying the distance of the fish to the stimulus dipole using "mean distance" would likely give unreliable result given that the length of the fish and the spread of the dipole are large relative to the size of the tank. Instead, the position of the fish was categorized in two zones: near or far. A square area of $21 \mathrm{~cm} \times 21 \mathrm{~cm}$ centered on the corner containing the dipole defined the near region, which was of similar area as the remainder of the tank, the far zone. The fish was considered located in the zone containing the majority of the pixels representing the fish. 
Results

In this study, three types of communication signals were used: low frequency beats (LFB: $10 \mathrm{~Hz}$ ) paired with small chirps (typical of agonistic encounters), high frequency beats (HFB: $120 \mathrm{~Hz}$ ) with big chirps and HFB with small chirps (both more typical of courtship). For each type, the stimulus was presented with several chirp variants, differing in duration and in frequency rise within a range typical of $A$. leptorhynchus signals. For each stimulus set, our

analyses were based on the responses of 15-20 cells (either ON-cells or OFF-cells) recorded from the LS. Superficial, intermediate, or deep cells were not targeted specifically, but baseline firing rates of $20.4 \mathrm{~Hz}$ ( \pm 9.6 s.d.) for ON-cells and $15.2 \mathrm{~Hz}$ ( \pm 7.5 s.d.) for OFF-cells, indicated that most recorded neurons were from superficial or intermediate layers (Bastian and Courtright, 1991). Using an analysis based on spike metric distances between neural responses (see Methods) we quantified the discrimination errors that an ideal decoder would make based on the information contained in these population responses. Efficient encoding should achieve low discrimination error rates based on the response of the fewest neurons possible.

\section{Encoding of signals mediating agonistic encounters does not efficiently represent chirp} properties.

Small chirp stimuli cause abrupt phase-shifts in the background beat cycle (Fig 1A). The spatial geometry of electric field and electroreceptors is such that a stimulus that increases electrical amplitude on one side of the body cause a decrease in amplitude on the other side, eliciting a response from ipsilateral ON-cells and OFF-cells respectively. As we have shown before (Marsat et al., 2009), superficial ON-cells of the lateral segment of the ELL respond with a more salient response than other cells in the ELL: they produce a high-frequency stereotyped burst of spikes after the chirps that serves as a particularly effective chirp-detection mechanism. Both the bursting and non-bursting responses contained some information about the AM shape 
of the chirp stimulus. However, these response types still led to error even when pooling the responses of 13 cells, with ON-cells performing significantly worse than OFF-cells (Fig 1B). The analysis in Figure 1B used the same procedure as Vonderschen and Chacron (2011) and the results are qualitatively similar. However, information about AM shape of a chirp did not allow an observer to discriminate differences in the parameters such as differences in duration or frequency rise (Fig 1C). For these stimuli, a chirp with specific duration and frequency occurring at a given phase will cause a different AM shape than the same chirp with identical frequency and duration properties occurring at another phase (compare the first and last stimuli in Fig 1A). Consequently, two chirps that differ in parameters the animal can control (duration, frequency) could elicit responses that are more similar to each other than to the response of identical chirps occurring at a different phases (which the animal does not control) (Aumentado-Armstrong et al., 2015). A decoder cannot rely on spiking pattern to estimate variation in these chirp signals since the relevant parameters are obscured by the phase on which the chirp occurs (Walz et al., 2013). Our results argue that the signal's characteristics hinder discrimination and this signal is thus encoded with a synchronized bursting code that is efficient for detection (Marsat et al., 2009) but less so for discrimination (Fig 1B). The characterization of the responses to these signals is the topic of a previous publication (Marsat et al., 2009), so we will not explore it in depth here. Not all neurons burst in response to these chirps, and the coding scheme we describe here is not crucially dependent on the neurons bursting, but rather on them having a relatively homogeneous response across chirps. Other studies indicate that these signals do indeed lead to correlated activity across the population, consistent with our findings (Metzen and Chacron, 2017). 
Big and small chirps occurring on high frequency beats span several cycles of the beat and thus the overall AM shape of the signals is virtually unaffected by the phase at which the chirp started (Waltz et al., 2014). Big chirps cause a transient increase in beat frequency that is also accompanied by a decrease in the beat amplitude directly proportional to the frequency increase (Zupanc and Maler, 1993). These chirps caused a graded increase in firing rate in OFFcells and a cessation of firing in ON-cells (Fig 2A). The increase in OFF-cell firing ranged from only a few $\mathrm{Hz}$ in some cells or a few hundred $\mathrm{Hz}$ in others (Marsat and Maler, 2010). For chirps that differ in duration and frequency, ON and OFF cells both allowed accurate discrimination based on the responses of a dozen cells (Fig 2B). The duration of the break in firing in ON-cells was proportional to the duration of the chirps, thus carrying information about chirp duration, but not frequency increase. Note that in some ON-cells, particularly superficial cells, the pause in firing was followed by an increase in firing rate that seems correlated, within a cell, to the duration of the pause. The role of this delayed aspect of the response is unclear and has not been explored further. When presented with two chirps that do not differ in duration but only in frequency, the pause in ON-cell firing cannot allow accurate discrimination. OFF-cells responded in a graded manner, increasing firing in relation to both chirp duration and frequency increase, allowing for efficient discrimination even when chirp duration is identical (Fig 2C). These data show that big chirps on high frequency beats are encoded with spiking patterns that allow the identification and discrimination of chirp characteristics.

Even though small chirps and big chirps are categorically different signals that often mediate different behaviors (Hagedorn and Heiligenberg, 1985; Hupé and Lewis, 2008), their behavioral impact (Dunlap and Larkins-Ford, 2003; Triefenbach and Zakon, 2008; Triefenbach and Zakon, 2003) and the way they are encoded in the electroreceptors (Benda et al., 2006) 
depends on the beat frequency. Specifically, in the electroreceptors, small chirps on lowfrequency beats cause an increase in synchrony among receptors, whereas both small-chirps and big-chirps on high frequency beats cause a decrease in synchrony (Walz et al., 2014). We see a similar separation of the responses in the ELL: the responses to small chirps on high frequency beats were more similar to big-chirp responses rather than to responses to small chirps on low frequency beats. OFF-cells responded with an increase in firing and ON-cells with a pause in firing (Fig 3A). The small chirps shown in Figure 3 caused an increase in firing of $20 \mathrm{~Hz}$ on average in OFF-cells, which corresponds to a doubling of baseline frequency. This increase appeared slightly stronger and longer in the larger of the small chirps. We calculated the instantaneous firing rate of population responses that include 12 neurons and averaged them across possible combinations and repeats (Fig 3B) to estimate the variability in the time course of the population response's firing rate. As described in the past for the response of OFF-cells to big chirps (Marsat and Maler, 2010), this population response was relatively invariant from trial to trial, therefore the difference in response shape for the two small chirps was larger than the typical variability in the response to a given chirp. Quantification of this observation in Figure 3C shows that discrimination error level based on spiking pattern differences reached very low values for OFF-cell population responses of 12 neurons or more. ON-cell responses have a difference in the time-course of the population instantaneous firing rate but it was small relative to the variability in the pattern of responses to the same stimulus and thus the discrimination remained inaccurate. It is possible that larger populations of responses (much more than 13 cells) could lead to error-less discrimination, presumably by partially averaging out the variability in the population response. Therefore, we conclude that both ON-cells and OFF-cells can support 
the discrimination of small chirps on high frequency beats but that OFF-cells do so more efficiently.

\section{Correlating behavior with coding strategy}

Our neurophysiological data argue for a match between a signal with a structure that hinders discrimination (small chirps LFB) and a neural code that is not efficient at supporting discrimination (Fig 1B) but rather geared towards sensitive detection of the stimulus (synchronized bursting). In contrast, signals with structures well suited to being discriminated are encoded with graded heterogeneous responses that efficiently carry information about chirp characteristics thus allowing discrimination of chirp variations. Despite this compelling evidence that neural codes and signal structures are matched to support different tasks, discriminating versus simply detecting the signals, it is unclear whether it actually mediates different behavioral responses and perceptual tasks. The neurophysiological results predict that some stimuli can be discriminated but not others; therefore, we tested the fish's ability to discriminate. We specifically hypothesized that the fish will not discriminate between different small chirps on LFB but that small chirps on HFB will be discriminated, as will big chirps on HFB.

To test this prediction, we used a paradigm that has been used successfully in a wide variety of animals and modalities to test perceptual discrimination abilities: a habituationdishabituation assay (Carlson et al., 2011; Cheney and Seyfarth, 1988; Miller-Sims and Bottjer, 2012; Penn and Potts, 1998; Wyttenbach et al.; Zuberbühler et al., 1999). The test relies on the fact that animals respond most strongly to novel stimuli and habituate to a stimulus that is presented, unchanged, repeatedly. In the steady, habituated state, the animal responds relatively weakly or not at all to the stimulus. When presented with a novel stimulus the animal displays a resurgence of response that reflects the fact that the animal perceives the stimulus as novel. This 
dishabituation response -or lack thereof- demonstrates that the animal discriminated -or notbetween the first and second stimulus. One of the strengths of this assay is that it takes advantage of the animal's innate response to a stimulus. The test therefore reveals how perceptual abilities are used to guide innate behaviors. In this case, sensory information about a stimulus' novelty is used to drive an increase in behavioral response. We took advantage of the fact that most $A$. leptorhynchus fish placed in a confined space (here, an aquarium of $30 \mathrm{~cm} \times 30 \mathrm{~cm} \times 10 \mathrm{~cm}$ ) will react to a conspecific signal by increasing swimming movement and speed, swimming around the source of the stimulus as if "investigating" and sometimes chirping, biting or lunging in that direction (Fig 4A). Our repeated stimulation protocol (Fig 4B, see methods for details) elicited responses that habituated and reached a steady state within 60-70 min. Therefore, after $90 \mathrm{~min}$ of stimulation with a given chirp, the stimulus was switched to a stimulus with the same beat frequency (HFB vs LFB) and the same chirp type (small vs big) but different chirp parameters (frequency rise or duration). We used the same stimuli as in the neurophysiological experiments.

\section{Swimming speed changes reveal perceptual discrimination of chirps on high frequency beats}

Various aspects of the behavioral response were quantified and several showed some habituation-dishabituation effect (biting, lunges, chirping; data not shown). Counting lunges or chirps clearly showed habituation, but only revealed dishabituation in a small subset of individuals. If the dishabituation response was not strong enough to cross the threshold where it would produce one of these highly aggressive behaviors the measure would not reveal the phenomenon. We found swimming speed and changing distance from the stimulus source were more sensitive measures of dishabituation.

The distribution of swimming speeds during a given stimulation bout typically had a bimodal form with a peak at slower swimming speed indicative of a passive state and the faster 
swimming speed occurring during active swimming (Fig 4C). We took the ratio of time spent in these two states (normalized for each fish) to quantify the strength of the behavioral response (Fig 4D, see Methods for details). The ratio was higher than 1 at the beginning of the assay when the fish reacted most strongly (i.e. swam faster) to the stimulus and also if the fish exhibited dishabituation to the novel stimulus.

Swimming speed decreased markedly in the first hour of stimulation (Fig 5A). There was no significant difference in average swimming speeds across stimulus types at the beginning of the stimulation protocol (ANOVA for time 0 of chirp $\mathrm{X}$ stimulation: $\mathrm{p}=0.3$ ) and, by design, reach the same speed ratio of 1 by the end of the habituation period. Although the change in speed across the habituation period was very similar across the three stimuli, the fish might have habituated slightly slower to low-frequency beats. This observation is supported by the fact that a few data-points around $45 \mathrm{~min}$ are significantly higher for the LFB stimulus compared to big chirps HFB for Fig 5A or small chirps HFB in Fig 5B (MANOVA followed by Tukey HSD with significance set at $\mathrm{p}<0.05)$.

The first stimulus bout with a new chirp led to a small increase in speed ratio for both small and big chirps on HFB but not for small chirps on LFB (MANOVA followed by Tukey HSD showed $0.15<\mathrm{p}<0.32$ between the high frequency beat stimuli at time 0 of chirp $\mathrm{Y}$ and the last three stimulus presentations of chirp X. For low frequency beat stimuli, the same comparison gave $0.36<p<0.42$ ). The small increase for high frequency beats was not significant because two factors affect the magnitude of this dishabituation effect: 1- The dishabituation response is marked in the first few seconds of stimulation but disappears quickly. Since each data point in Figure $5 \mathrm{~A}$ is an average across the whole $1 \mathrm{~min}$ of stimulation, the short dishabituation effect did not influence the values very much; 2- dishabituation was observed more clearly in some fish 
than others as a function of the position of the fish when the new stimulus starts (see next paragraph and Fig 5C). To account for the fact that behavioral responses were the strongest at the beginning of each 1 min stimulation bouts and that the dishabituation effect was short-lived, we quantified speed ratios in the first 5 seconds of each bout (Fig 5B). These behavioral responses show a similar habituation trend that plateaued after 60 minutes. The dishabituation when the new stimulus was played was obvious and significant for chirps on high frequency beats but not for small chirps on low frequency beats (MANOVA followed by Tukey HSD showed $0.02<\mathrm{p}<0.04$ between the HFB stimuli at time 0 of chirp Y and the last three stimulus presentations of chirp X. For LFB stimuli, the same comparisons give $0.27<p<0.45$ ).

\section{Reaction of the fish to a novel stimulus depends on its distance to the stimulus source}

When trying to identify the factors that could influence the variability in the observed behavior, we noticed that some fish did not show a recovery of active swimming when the new stimulus was played. In every case, the fish was close to the stimulation electrode just prior to the start of the new stimulation. In contrast, fish that were further from the electrode reliably started to move actively towards the stimulation electrode when a new chirp on HFB was delivered. We quantified this by separating the trials based on the position of the fish in the 1 second prior to the start of the new stimulus. Trials where the fish had over $50 \%$ of its body length in the "far" zone (Fig 4A) were categorized as such whereas in the other trials the fish were defined as being "near". We contrasted the speed ratio in the three stimulation bouts prestimulus-change (only the first 5 seconds were taken in account as in Fig 5B) to the swimming speed in the first seconds of post-chirp-change stimulation. Our data confirm that dishabituation was observable only if the fish was not in close proximity to the stimulation electrode (Fig 5C, paired t-test, $p<0.01$ for the HFB stimuli with the fish classified as "far" and $p>0.1$ for all 
others). We speculate that fish that were located near the electrodes when the new stimulus was introduced could determine there were no other changes associated with the stimulus (e.g. another fish getting closer) and thus it did not need to get closer and investigate. Fish approached the novel stimulus thus showing discrimination of chirps only on high frequency beats

Position in the tank could also reveal changes in behavioral responsiveness of the fish if the fish remained near the stimulus source when actively engaged by the stimulus and less so when the behavioral response habituated. We categorized the position of the fish based on zones (see Fig 4A and Methods), although we repeated our analysis with other ways of quantifying distance (e.g. head to middle-of-dipole distance) with qualitatively similar results. The overall fish position did not change as the fish habituated to the stimulus (Fig 6A). When swimming actively, the fish often directed its movement towards the electrodes but also swam to-and-fro and often circled the stimulation zone, which resulted in a relatively spread distribution of position overlapping both near and far zones. When responding less strongly (e.g. in habituated state) the fish often remained still on one of the sides of the tank. Each fish had its preference as to which side it chose (near or far from the electrodes), leading to a broad distribution of positions. Nevertheless, by selecting the first five seconds of each stimulus and categorizing the trials based on position just prior to the new stimulus (as in Fig 5C) we saw a change in the position of the fish in response to changes in HFB stimuli (Fig 6B). This is consistent with the scenario that led to the results in Figure 5C: in the first few seconds of a new high frequency beat stimulus, if the fish was far from the electrode it swam towards the electrode and passed over it several times presumably to investigate the source of the new stimulus. 


\section{Discussion}

Our data demonstrate a clear link between the perceptual responses to a communication signal and the neural response patterns encoding that signal. Our electrophysiological recordings show that two discrete patterns of firing encode chirps: one code with high information content (heterogeneous and graded changes in firing) about chirp details and one with low information content about chirp properties (synchronous bursting). The behavioral response of the fish shows that these two codes mediate different perceptual tasks. The fish tested were able to perceive and react to slight differences in chirps that are encoded via grade heterogeneous firing rates. Chirp stimuli that elicited stereotyped bursts, however, did not result in discrimination behavior. This change in coding strategy is mediated by the beat frequency on which the chirp is presented. This is not simply a consequence of the chirp characteristics itself but also depends on the background beat which sets the context: an individual with similar EOD frequency (i.e. most likely an individual of similar sex and maturity) or not.

\section{Neural coding is matched to signal structure}

Small chirps occurring on low frequency beats cause large shifts in the background beat, but vary dramatically in the shape of the electrical image depending on the phase of the beat they occur on. This phase variance masks properties of small chirps such as duration and frequency rise. Emitting fish do not actively control which phase of the beat they chirp on (AumentadoArmstrong et al., 2015; Walz et al., 2013). Likewise, a given small chirp presented at different phases is encoded in an invariant manner in the midbrain and elicits invariant behavioral responses in the receiving fish (Metzen et al., 2016). The lack of phase control in chirp behavior, and invariance of response in the physiology demonstrates that phase is most likely not relevant 
to either the emitting or receiving fish. It does, however, actively hinder the ability to discriminate chirp properties.

The transient disruptions caused by a small chirp on the slow ongoing background frequency cause a brief synchronization in the firing or quiescence of the electroreceptor afferents (Benda et al., 2005; Benda et al., 2006). Likewise, pyramidal cells respond with a stereotyped (Marsat et al., 2009) and highly correlated responses across the population (Metzen et al., 2016). The brief durations of small chirps in conjunction with the long cycles of slow frequency beats drastically impairs discrimination of chirp parameters such as duration and frequency. Our data confirm the results of Marsat and Maler (2010) demonstrating that responses to small chirps on low frequency beats cannot be discriminated from one another by a biologically realistic decoding mechanism.

Since we performed single-cell recording rather than multi-unit recordings, we cannot take in account the effect of noise correlation on population coding. For spatially diffuse signals such as communication signals, the amount of noise correlation has been shown to be relatively small (Simmonds and Chacron, 2015) and limited to neurons with overlapping receptive fields (Chacron and Bastian, 2008). We therefore expect the influence of noise correlations to be small. Furthermore, noise correlations would most likely deteriorate coding further for a population of pyramidal cells of a single -ON or OFF- type since their stimulus-driven response will be correlated (for a thorough discussion of noise correlations see: Averbeck et al., 2006). Most importantly, no effect of noise correlations can circumvent the main factor that prevents these signals from being discriminated: the fact that the signal itself impede discrimination as explained above. 
At higher beat frequencies both big and small chirps span more than one beat cycle, reducing the effect of phase on the electrical image the fish receives. This change in the signal structure allow the system to encode details about chirp duration and frequency. At these beats frequencies, electroreceptor afferents phase lock more synchronously to the beat than to the chirps; both small and big chirps disrupt that synchrony (Benda et al., 2006; Walz et al., 2014). Desynchronization in afferent firing gives rise to the observed ON-cell inhibition and variable increase in OFF-cell firing that occurs in response to both chirp types on high frequency beats. The variability of the response to chirps on high frequency beats was observed first for big chirps (Marsat and Maler, 2010) and confirmed for small chirps (Metzen and Chacron, 2017). This graded OFF-cell response provides sufficient information for a decoder to accurately discriminate between big chirps. Here, we demonstrate that this coding strategy is not specific to big chirps, but is also observed for small chirps. The change in chirp structure and, as a result, the change in coding, is a product of the social context (beat frequency) rather than due to the properties of the chirp itself.

It should also be noted that, to date, all studies of chirp responses in the ELL have been undertaken using stationary stimuli. It is very possible that including the effects of movement may make the tasks of detection and discrimination more difficult since it would modulate the spatial and spectral characteristics of the stimulus (Fotowat et al., 2013; Kelly et al., 2008). Furthermore, it is known that movement can affect pyramidal cell responses (e.g. Clarke et al., 2014). Further studies using spatially realistic and dynamic stimuli might thus provide additional insight. 


\section{Perceptual ability corresponds to coding strategy}

What could be the advantage of using two different coding strategies for the different contexts? Our results argue for the fact that the type of neural codes used in different situations should be matched to the perceptual task being performed. In diverse sensory systems including vision, audition, and touch, there are demonstrable links between the stimulus encoding strategy and the animal's sensory acuity (Adibi and Arabzadeh, 2011; Arabzadeh et al., 2003; Bendor and Wang, 2007; von Heimendahl et al., 2007). Our results systemically correlate two different neural codes with specific behavioral tasks: the ability to discriminate versus simply detecting the stimulus. Since our behavioral test measures motor output and not perception directly, we cannot exclude the possibility that fish perceived a difference in small chirps on low frequency beats but did not react. However, it does not affect the strength of our conclusion. The fact that the behavioral response is determined by the presence or absence of the chirp -not by its detailed characteristics- demonstrates that the behavior is guided by chirp detection. Whether the information to discriminate is present at one point in the nervous system is irrelevant, as this information does not influence the behavior we examined. Our results suggest that different neural codes might be used in order to perform each the two different behavioral tasks more efficiently. The results thus lead to the prediction that, in this system, there is a tradeoff between sensitive detection and accurate discrimination and coding efficiency is maintained by changing coding scheme.

\section{Specialization of coding for separate tasks}

In A. leptorhynchus beat frequency establishes the social context of conspecific interaction and may function as a filter, priming the ELL for efficient, task-dependent signal 
processing. Different sub-populations of cells best encode the signals to be detected versus the signals to be discriminated ( ON-cells vs. OFF-cells respectively; Marsat and Maler, 2010; Marsat et al., 2009). However, these studies and our current results also demonstrate that both subpopulations' responses allow detection and discrimination. Therefore, the difference in processing is not mainly a difference in the subpopulation that carries out the task, but rather a difference in response pattern and stimulus shape. In other words, the neural coding strategy changes as a function of the stimulus. Ollerenshaw et al. (2014) demonstrated in the rodent vibrissae system that adaptation changes the neural code from one primed for detection to one better able to discriminate - a change mirrored in behavioral performance. In the present case, changes in coding are not implemented by adaptation but are a consequence of the fixed properties of the network such as feedforward frequency tuning (Walz et al., 2014) and feedback (Marsat et al., 2012). As indicated by our results, and studies of coding strategies in diverse sensory systems (e.g. visual: Lesica, Nicholas, Stanley, 2004; Sherman, 2001; insect auditory: Marsat and Pollack, 2004; Sabourin and Pollack, 2010), the use of different neural coding strategies for detection and discrimination tasks might be a wide-spread phenomenon that allows efficient coding in a constantly changing environment.

Burst firing is a particularly robust method of encoding sensory information (Krahe and Gabbiani, 2004). Bursting is not an essential requirement of this coding scheme, which is characterized by highly correlated activity across the population and invariant responses across stimuli. Responses to small chirps of low-frequency beats possess both these characteristics (Marsat et al., 2009; Metzen and Chacron, 2017; Metzen et al., 2016). However, the bursting dynamic increases the gain for certain stimuli (Marsat and Maler, 2012; Mehaffey et al., 2005) and thus increases the saliency of the response. A feature detection coding scheme is especially 
useful when encoding transient, but important, communication signals (Marsat et al., 2009) or small prey items (Oswald et al., 2004). In this light, the stereotyped burst response produced by small chirps on low frequency beats may reflect their ethological significance to the animal. On low frequency beats, indicative of same sex interactions between closely sized animals, small chirps are most commonly produced during agonistic encounters (Hupé and Lewis, 2008). Behavioral studies indicate that chirp timing is a key determinant of the interaction they mediate (Hupé, 2012). Presumably, the chirp emission pattern rather than the detailed structure of each chirp carries the information to guide behavioral responses. Detailed discrimination of these chirps may be sacrificed for reliability in detection.

The rational for needing an efficient detection code specifically for small chirps on low frequency beats has not been experimentally determined yet. In the wild, small chirps are used in various contexts (Henninger, 2015; Henninger et al., 2017). They are often produced at close range (Zupanc et al., 2006), but can be exchanged between individuals as far as $30 \mathrm{~cm}$ away (Henninger, 2015; Henninger et al., 2017) or more (Zupanc et al., 2006; see their Fig 3). At distances of $20-30 \mathrm{~cm}$, the beat contrast (i.e. effective intensity of the stimulus) can be as low as 1\% (Fotowat et al., 2013). Other signals and sources of noise (e.g. another nearby fish) can also add to the sensory stream. A chirp in this weak signal, possibly embedded in noise is likely to activate the sensory system only weakly and thus a sensitive detection mechanism might be beneficial.

Burst structure is stereotyped, and in large part dictated by the burst mechanism. While some stimulus features may be extracted from bursts (e.g. Marsat and Pollack, 2010; MartinezConde et al., 2002; Oswald et al., 2007) they are limited in the amount of information they can encode in their ISI due to their stereotyped structure. Discrimination tasks therefore benefit from 
an alternate form of coding. Highly variable population responses with graded heterogeneous responses are well suited for that purpose since they accomplish signal whitening (Marsat and Maler, 2010) and thus maximize channel capacity (Doi and Lewicki, 2014; Simoncelli and Olshausen, 2001). The heterogeneity of the population has been highlighted in several publications (Bastian and Nguyenkim, 2001; Ly and Marsat, 2017; Metzen and Chacron, 2015; Sproule et al., 2017). The heterogeneous responses of the population may sacrifice the reliability of detection through burst for the detailed coding of chirp features. Supporting the idea that chirp detectability decreases when coding switches from a feature detection code to a graded one, Metzen and Chacron (2017) showed that detectability -or at the very least response to- small chirps decrease when the background beat is changed from low to high frequency. It is currently unknown whether chirp parameters influence behaviors such as courtship. However, given the range of parameters in chirps produced by these fish, it is not unrealistic to hypothesize that these variations carry behaviorally relevant information. Therefore, using a neural code efficient for discrimination could be advantageous in contexts where chirp structure can carry information

In conclusion, our data show that the perceptual ability of an animal can be linked to the use of specific coding strategies. The ability to discriminate between chirps corresponds with a graded, heterogeneous neural response that is high in information about chirp structure, while a bursting code consisting of highly stereotyped responses is used for coding signals that are not behaviorally discriminated. These two coding strategies appear frequently across many sensory systems, implying that the specialization of these two neural codes for discrimination vs. detection tasks is a common phenomenon in neural system. 
References

Adibi, M. and Arabzadeh, E. (2011). A comparison of neuronal and behavioral detection and discrimination performances in rat whisker system. J. Neurophysiol. 105, 356-65.

Arabzadeh, E., Petersen, R. S. and Diamond, M. E. (2003). Encoding of Whisker Vibration by Rat Barrel Cortex Neurons: Implications for Texture Discrimination. Jounal Neurosci. 23, 9146-9154.

Aumentado-Armstrong, T., Metzen, M. G., Sproule, M. K. J. and Chacron, M. J. (2015). Electrosensory Midbrain Neurons Display Feature Invariant Responses to Natural Communication Stimuli. PLoS Comput. Biol. 11, 1-28.

Averbeck, B. B., Latham, P. E. and Pouget, A. (2006). Neural correlations, population coding and computation. Nat. Rev. Neurosci. 7, 358-366.

Barlow, H. B. (1961). Possible principles underlying the transformations of sensory messages. In Sensory Communication (ed. Rosenblith, W. A.), pp. 217-234. Cambridge, MA: MIT Press.

Bastian, J. and Courtright, J. (1991). Morphological correlates of pyramidal cell adaptation rate in the electrosensory lateral line lobe of weakly electric fish. J. Comp. Physiol. A 168, 393407.

Bastian, J. and Nguyenkim, J. (2001). Dendritic modulation of burst-like firing in sensory neurons. J. Neurophysiol. 85, 10-22.

Bastian, J., Schniederjan, S. and Nguyenkim, J. (2001). Arginine Vasotocin Modulates a Sexually Dimorphic Communication Behavior in the Weakly Electric fish Apteronotus leptorhynchus. J. Exp. Biol. 204, 1909-1923.

Benda, J., Longtin, A. and Maler, L. (2005). Spike-frequency adaptation separates transient communication signals from background oscillations. J. Neurosci. 25, 2312-21.

Benda, J., Longtin, A. and Maler, L. (2006). A synchronization-desynchronization code for natural communication signals. Neuron 52, 347-58.

Bendor, D. and Wang, X. (2007). Differential neural coding of acoustic flutter within primate auditory cortex. Nat. Neurosci. 10, 763-71.

Bradbury, J. W. and Vehrencamp, S. L. (2011). Principles of Animal Communication. 2nd ed. Sunderland, Mass.: Sunderland: Sinauer Associates.

Carlson, B. A., Hasan, S. M., Hollmann, M., Miller, D. B., Harmon, L. J. and Arnegard, M. E. (2011). Brain Evolution Triggers Increased Diversification of Electric Fishes. Science (80-. ). $332,583-586$.

Chacron, M. J. and Bastian, J. (2008). Population coding by electrosensory neurons. J. Neurophysiol. 99, 1825-1835. 
Cheney, D. L. and Seyfarth, R. M. (1988). Assessment of meaning and the detection of unreliable signals by vervet monkeys. Anim. Behav. 36, 477-486.

Clarke, S. E., Longtin, A. and Maler, L. (2014). A Neural Code for Looming and Receding Motion Is Distributed over a Population of Electrosensory ON and OFF Contrast Cells. J. Neurosci. 34, 5583-5594.

Doi, E. and Lewicki, M. S. (2014). A Simple Model of Optimal Population Coding for Sensory Systems. PLoS Comput. Biol. 10,.

Dunlap, K. D. and Larkins-Ford, J. (2003). Production of aggressive electrocommunication signals to progressively realistic social stimuli in male Apteronotus leptorhynchus. Ethology 109, 243-258.

Fairhall, A. L., Lewen, G. D., Bialek, W. and de Ruyter Van Steveninck, R. R. (2001). Efficiency and ambiguity in an adaptive neural code. Nature 412, 787-92.

Fotowat, H., Harrison, R. R. and Krahe, R. (2013). Statistics of the Electrosensory Input in the Freely Swimming Weakly Electric Fish Apteronotus leptorhynchus. J. Neurosci. 33, 1375813772.

Freedman, D. J., Riesenhuber, M., Poggio, T. and Miller, E. K. (2001). Categorical representation of visual stimuli in the primate prefrontal cortex. Science (80-. ). 291, 3126.

Hagedorn, M. and Heiligenberg, W. (1985). Court and spark: electric signals in the courtship and mating of gymnotoid fish. Anim. Behav. 33, 254-265.

Henninger, J. (2015). Social Interactions in Natural populations of weakly electric fish.

Henninger, J., Kirschbaum, F., Grewe, J., Krahe, R. and Benda, J. (2017). Sensory tuning does not match behavioral relevance of communication signals in free-living weakly electric fish. bioRxiv DOI, 10.1101/114249.

Hupé, G. J. (2012). Electrocommunication in a Species of Weakly Electric Fish Apteronotus Leptorhynchus: Signal Patterning and Behaviour.

Hupé, G. J. and Lewis, J. E. (2008). Electrocommunication signals in free swimming brown ghost knifefish, Apteronotus leptorhynchus. J. Exp. Biol. 211, 1657-1667.

Kelly, M., Babineau, D., Longtin, A. and Lewis, J. E. (2008). Electric field interactions in pair of electric fish: modeling and mimicking naturalistic inputs. Biol. Cybern. 98, 479-490.

Krahe, R. and Gabbiani, F. (2004). Burst firing in sensory systems. Nat. Rev. Neurosci. 5, 13-23.

Kröger, B. J., Birkholz, P., Kannampuzha, J. and Neuschaefer-Rube, C. (2011). Categorical perception of consonants and vowels: Evidence from a neurophonetic model of speech production and perception. Lect. Notes Comput. Sci. (including Subser. Lect. Notes Artif. Intell. Lect. Notes Bioinformatics) 6456 LNCS, 354-361.

Larson, E., Billimoria, C. P. and Sen, K. (2009). A biologically plausible computational model for auditory object recognition. J. Neurophysiol. 101, 323-31. 
Laubach, M., Wessberg, J. and Nicolelis, M. A. (2000). Cortical ensemble activity increasingly predicts behaviour outcomes during learning of a motor task. Nature 405, 567-71.

Lesica, Nicholas A., Stanley, G. B. (2004). Encoding of Natural Scene Movies by Tonic and Burst Spikes in the Lateral Geniculate Nucleus. J. Neurosci. 24, 10731-10740.

Ly, C. and Marsat, G. (2016). Variable Synaptic Strengths Controls the Firing Rate Distribution in Feedforward Neural Networks. Pre-print.

Machens, C. K., Schütze, H., Franz, A., Kolesnikova, O., Stemmler, M. B., Ronacher, B. and Herz, A. V. M. (2003). Single auditory neurons rapidly discriminate conspecific communication signals. Nat. Neurosci. 6, 341-342.

Maler, L., Sas, E. K. B. and Rogers, J. (1981). The cytology of the posterior lateral line lobe of high-frequency weakly electric fish (Gymnotidae) - Dendritic differentiation and synaptic specificity in a simple cortex. J. Comp. Neurol. 195, 87-139.

Maler, L., Sas, E., Johnston, S. and Ellis, W. (1991). An atlas of the brain of the electric fish Apteronotus leptorhynchus. J. Chem. Neuroanat. 4, 1-38.

Marsat, G. and Maler, L. (2010). Neural heterogeneity and efficient population codes for communication signals. J. Neurophysiol. 104, 2543-55.

Marsat, G. and Maler, L. (2012). Preparing for the unpredictable: adaptive feedback enhances the response to unexpected communication signals. J. Neurophysiol. 107, 1241-6.

Marsat, G. and Pollack, G. S. (2004). Differential temporal coding of rhythmically diverse acoustic signals by a single interneuron. J. Neurophysiol. 92, 939-48.

Marsat, G. and Pollack, G. S. (2010). The structure and size of sensory bursts encode stimulus information but only size affects behavior. J. Comp. Physiol. A Neuroethol. Sensory, Neural, Behav. Physiol. 196, 315-320.

Marsat, G. and Pollack, G. S. (2012). Bursting neurons and ultrasound avoidance in crickets. Front. Neurosci. 6, 1-9.

Marsat, G., Proville, R. D. and Maler, L. (2009). Transient signals trigger synchronous bursts in an identified population of neurons. J. Neurophysiol. 102, 714-23.

Marsat, G., Longtin, A. and Maler, L. (2012). Cellular and circuit properties supporting different sensory coding strategies in electric fish and other systems. Curr. Opin. Neurobiol. 22, 686692.

Marsat, G., Hupé, G. J. and Allen, K. M. (2014). Heterogeneous response properties in a population of sensory neurons are structured to efficiently code naturalistic stimuli. In Society for Neuroscience Conference, p. 181.20.

Martinez-Conde, S., Macknik, S. L. and Hubel, D. H. (2002). The function of bursts of spikes during visual fixation in the awake primate lateral geniculate nucleus and primary visual cortex. Proc. Natl. Acad. Sci. U. S. A. 99, 13920-13925.

Mehaffey, W. H., Doiron, B., Maler, L. and Turner, R. W. (2005). Deterministic multiplicative 
gain control with active dendrites. J. Neurosci. 25, 9968-9977.

Metzen, M. G. and Chacron, M. J. (2015). Neural heterogeneities determine response characteristics to second-, but not first-order stimulus features. J. Neurosci. 35, 3124-38.

Metzen, M. G. and Chacron, M. J. (2017). Stimulus background influences phase invariant coding by correlated neural activity. Elife 6,.

Metzen, M. G., Hofmann, V. and Chacron, M. J. (2016). Neural correlations enable invariant coding and perception of natural stimuli in weakly electric fish. Elife 5, 3-5.

Miller-Sims, V. C. and Bottjer, S. W. (2012). Development of Auditory-Vocal Perceptual Skills in Songbirds. PLoS One 7,.

Mitchell, B. R., Makagon, M. M., Jaeger, M. M. and Barrett, R. H. (2006). Information content of coyote barks and howls. Bioacoustics 15, 289-314.

Moore, C. I. (2004). Frequency-dependent processing in the vibrissa sensory system. J. Neurophysiol. 91, 2390-9.

Oglesbee, E. and Kewley-Port, D. (2009). Estimating vowel formant discrimination thresholds using a single-interval classification task. J. Acoust. Soc. Am. 125, 2323-35.

Ollerenshaw, D. R., Zheng, H. J. V, Millard, D. C., Wang, Q. and Stanley, G. B. (2014). The adaptive trade-off between detection and discrimination in cortical representations and behavior. Neuron 81, 1152-64.

Oswald, A.-M. M., Chacron, M. J., Doiron, B., Bastian, J. and Maler, L. (2004). Parallel processing of sensory input by bursts and isolated spikes. J. Neurosci. 24, 4351-62.

Oswald, A.-M. M. M., Doiron, B. and Maler, L. (2007). Interval coding. I. Burst interspike intervals as indicators of stimulus intensity. J. Neurophysiol. 97, 2731-2743.

Panzeri, S., Macke, J. H., Gross, J. and Kayser, C. (2015). Neural population coding: combining insights from microscopic and mass signals. Trends Cogn. Sci. 19, 162-72.

Penn, D. and Potts, W. K. (1998). Untrained mice discriminate MHC-determined odors. Physiol. Behav. 64, 235-243.

Richards, D. G. (1981). Alerting and Message Components in Songs of Rufous-Sided Towhees. Behaviour 76, 223-249.

Sabourin, P. and Pollack, G. S. (2010). Temporal coding by populations of auditory receptor neurons. J. Neurophysiol. 103, 1614-21.

Saunders, J. and Bastian, J. (1984). The physiology and morphology of two types of electrosensory neurons in the weakly electric fish Apteronotus leptorhynchus. J. Comp. Physiol. A 154, 199-209.

Sharpee, T., Destexhe, A., Kawato, M., Sekulić, V., Skinner, F. K., Wójcik, D. K., Chintaluri, C., Cserpán, D., Somogyvári, Z., Kim, J. K., et al. (2016). 25th Annual Computational Neuroscience Meeting: CNS-2016. BMC Neurosci. 17, P195. 
Sherman, S. M. (2001). Tonic and burst firing: dual modes of thalamocortical relay. Trends Neurosci. 24, 122-126.

Simmonds, B. and Chacron, M. J. (2015). Activation of parallel fiber feedback by spatially diffuse stimuli reduces signal and noise correlations via independent mechanisms in a cerebellum-like structure. PLoS Comput. Biol. 11, e1004034.

Simoncelli, E. P. and Olshausen, B. A. (2001). Natural image statistics and neural representation. Annu. Rev. Neurosci. 24, 1193-1216.

Sproule, M. M. K. J., Chacron, M. M. J., Sproule, M. M. K. J., Chacron, M. M. J., Chacron, M. M. J. and Miller, E. (2017). Electrosensory neural responses to natural electro-communication stimuli are distributed along a continuum. PLoS One 12, e0175322.

Stecker, G. C., Harrington, I. A. and Middlebrooks, J. C. (2005). Location coding by opponent neural populations in the auditory cortex. PLoS Biol. 3, e78.

Tremblay, F., Ageranioti-Belanger, S. A. and Chapman, C. E. (1996). Cortical mechanisms underlying tactile discrimination in the monkey. I. Role of primary somatosensory cortex in passive texture discrimination. J Neurophysiol. 76, 3382-3403.

Triefenbach, F. and Zakon, H. (2003). Effects of sex, sensitivity and status on cue recognition in the weakly electric fish Apteronotus leptorhynchus. Anim. Behav. 65, 19-28.

Triefenbach, F. A. and Zakon, H. H. (2008). Changes in signalling during agonistic interactions between male weakly electric knifefish, Apteronotus leptorhynchus. Anim. Behav. 75, 1263-1272.

Tripathy, S. J., Padmanabhan, K., Gerkin, R. C. and Urban, N. N. (2013). Intermediate intrinsic diversity enhances neural population coding. Proc. Natl. Acad. Sci. U. S. A. 110, 8248-8253.

van Rossum, M. C. (2001). A novel spike distance. Neural Comput. 13, 751-763.

von Heimendahl, M., Itskov, P. M., Arabzadeh, E. and Diamond, M. E. (2007). Neuronal activity in rat barrel cortex underlying texture discrimination. PLOS Biol. 5, e305.

Vonderschen, K. and Chacron, M. J. (2011). Sparse and dense coding of natural stimuli by distinct midbrain neuron subpopulations in weakly electric fish. J. Neurophysiol. 106, 3102-18.

Walz, H., Hupe, G. J., Benda, J. and Lewis, J. E. (2013). The neuroethology of electrocommunication: How signal background influences sensory encoding and behaviour in Apteronotus leptorhynchus. J. Physiol. 107, 13-25.

Walz, H., Grewe, J. and Benda, J. (2014). Static frequency tuning accounts for changes in neural synchrony evoked by transient communication signals. J. Neurophysiol. 112, 752-65.

Wyttenbach, R. A., May, M. L. and Hoy, R. R. Categorical Perception of Sound Frequency by Crickets. Science (80-. ). 273, 1542-1544.

Zuberbühler, K., Cheney, D. L. and Seyfarth, R. M. (1999). Conceptual semantics in a Nonhuman Primate. J. Comp. Psychol. 113, 33-42. 
Zupanc, G. K. H. and Maler, L. (1993). Evoked chirping in the weakly electric fish Apteronotus leptorhynchus : a quantitative biophysical analysis. Can. J. Zool. 71, 2301-2310.

Zupanc, G. K. H., Sirbulescu, R. F., Nichols, A. and Ilies, I. (2006). Electric interactions through chirping behavior in the weakly electric fish, Apteronotus leptorhynchus. J. Comp. Physiol. A 192, 159-173. 
Figures

A
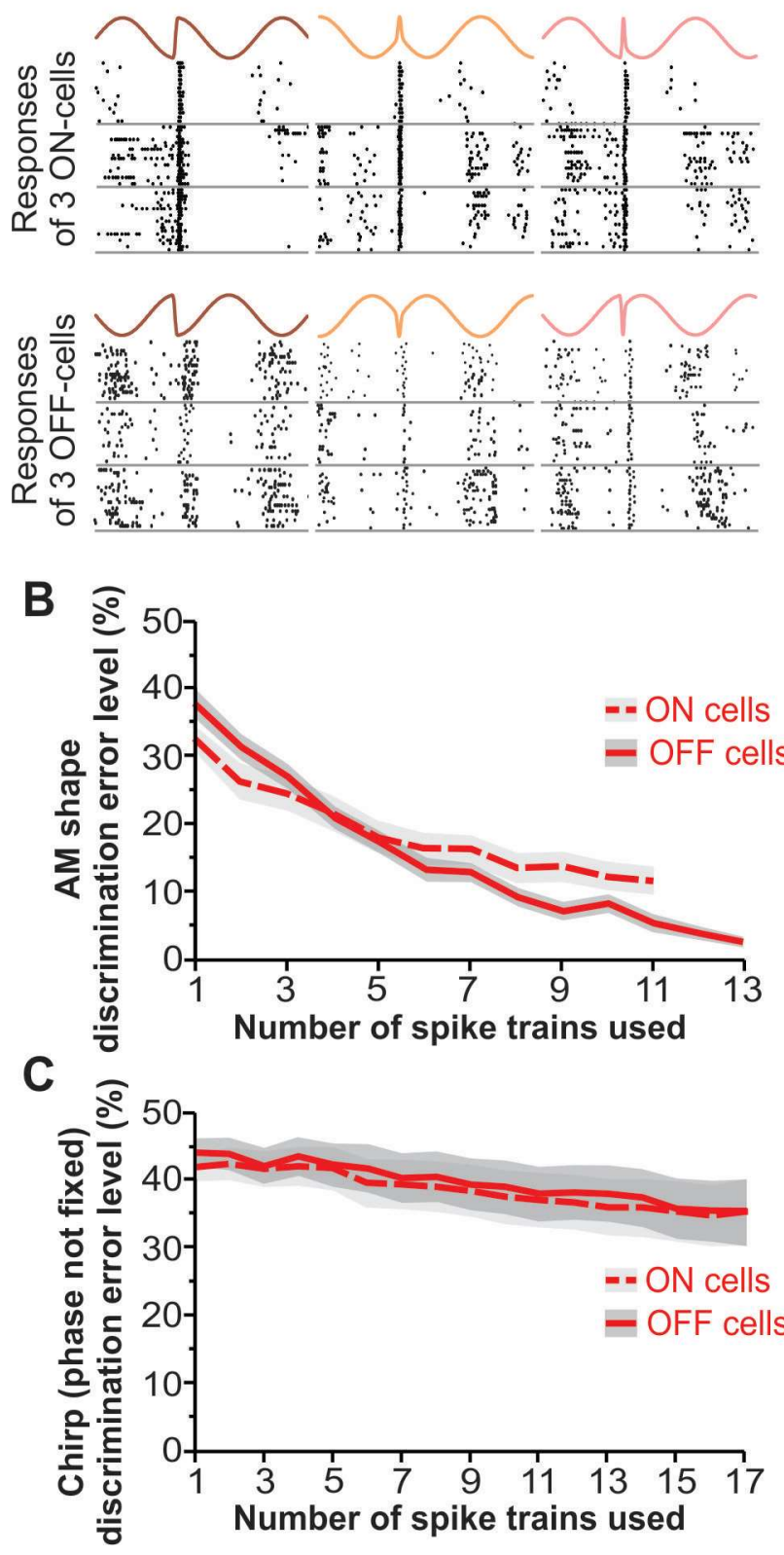

Figure 1:

Small chirps cannot be discriminated when presented on low frequency beats in a realistic manner. A. Example responses of 3 ON-cells (top) and 3 OFF-cells (bottom) stimulated with small chirps on a $10 \mathrm{~Hz}$ beat. The phase at which the chirp occurs changes the shape of the signal, even when the chirp frequency and duration are the same. Note that the chirp on the left and on the right have the same properties but are presented at different phases while the chirp in the middle differs in both duration and frequency. B. Discrimination analysis performed on spike train responses to chirps varying in both duration and frequency but occurring at the same phase. Shaded area indicates standard error. C. Discrimination accuracy for chirps with varying parameters when phase is not kept fixed. This scenario reflects the most natural aspects of chirp occurrence. Since chirps are not produced at specific phases discrimination of chirp features would have to happen despite the variability of starting phase. Error level remains close to chance-level (50\%) even when many spike trains are recruited. 
A Big chirps on high frequencies
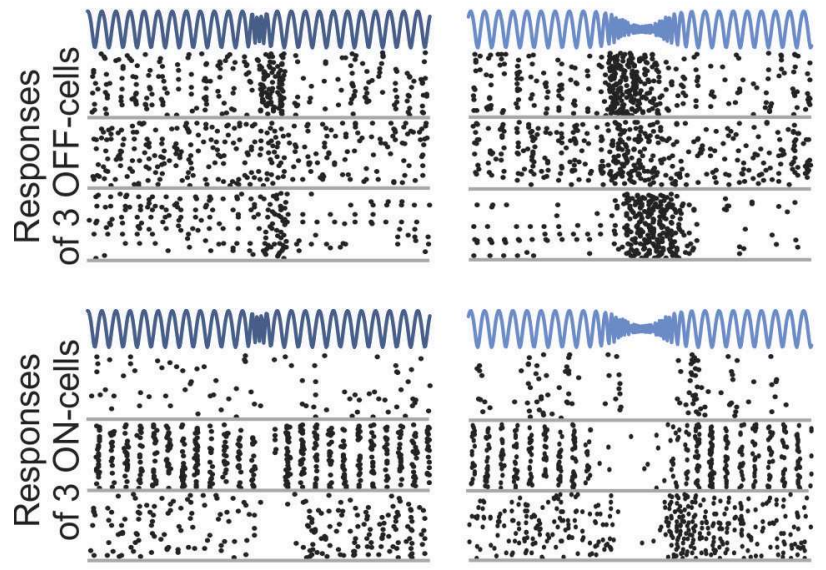

B

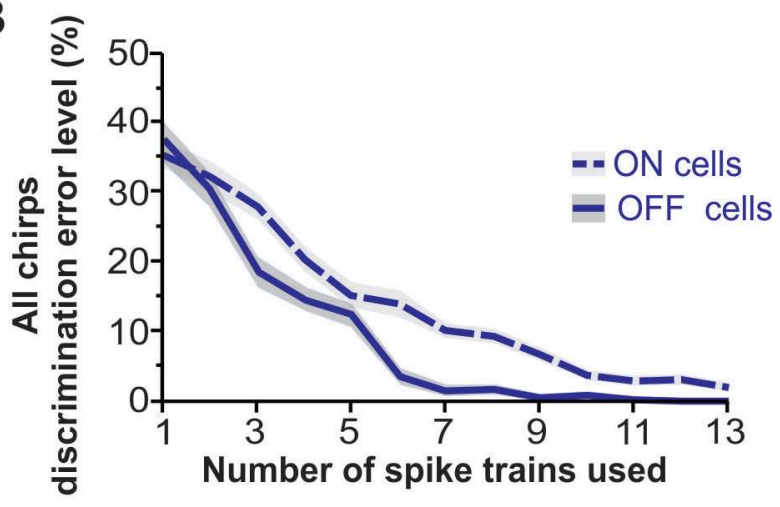

C

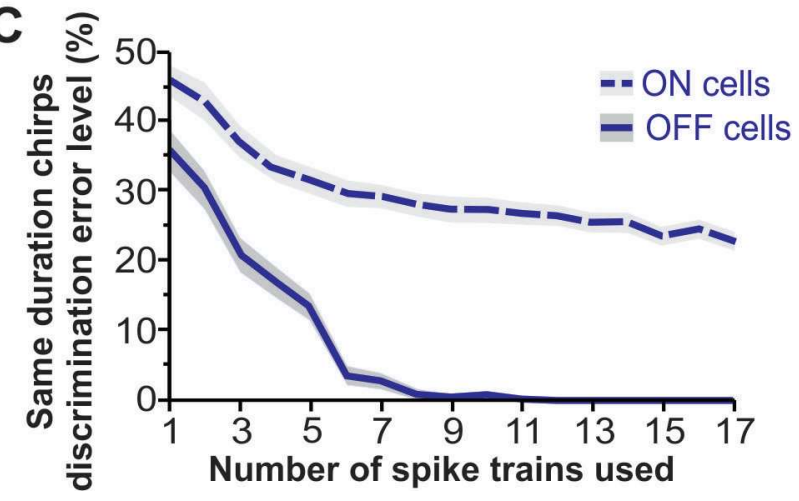

Figure 2:

Big chirps on high frequency beats are discriminated efficiently. A. Example responses of $3 \mathrm{ON}$ - and $3 \mathrm{OFF}$-cells to big chirps that vary in both frequency and duration and that occur on a $120 \mathrm{~Hz}$ beat. Big chirps variably increase OFF-cell firing rate. $\mathrm{ON}$ cells are inhibited by big chirps. B. Chirps that vary in both duration and frequency rise are discriminated by both ON- and OFF- cells. C. Chirps that vary only in frequency, but not duration, can only be discriminated by the graded responses of OFF-cells. Shaded area indicates standard error. 
A Two different small chirps on high frequency beats

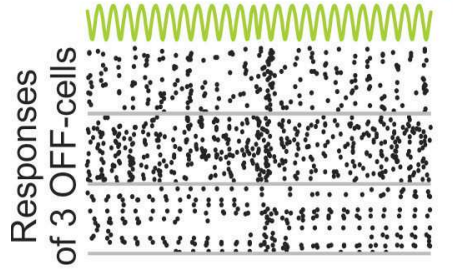

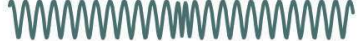

$\therefore$ a

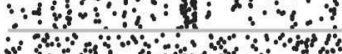

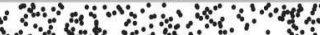

, mo of

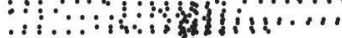

$\therefore \therefore$ :....

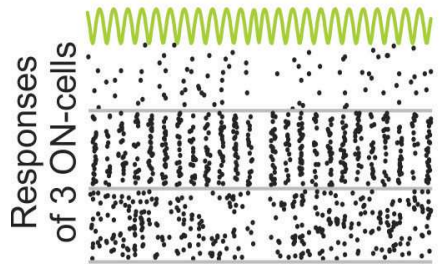

WMYMYMYMYMYMYY

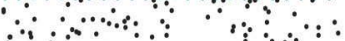

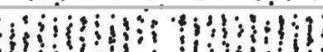

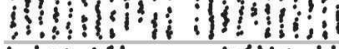

$\therefore$ a a a a

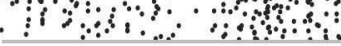

B

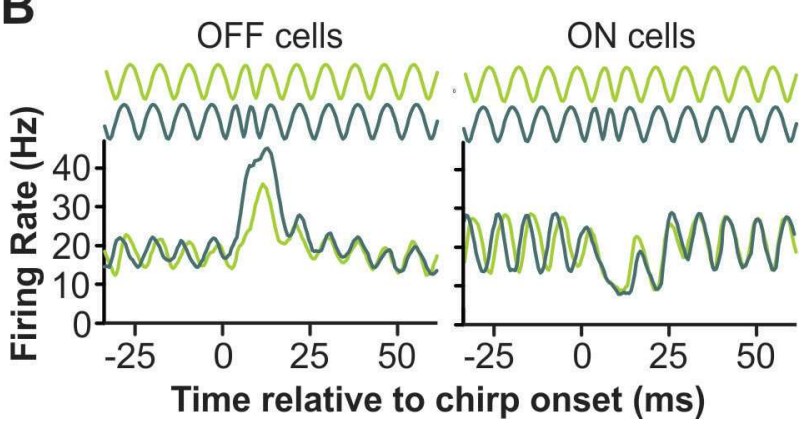

C

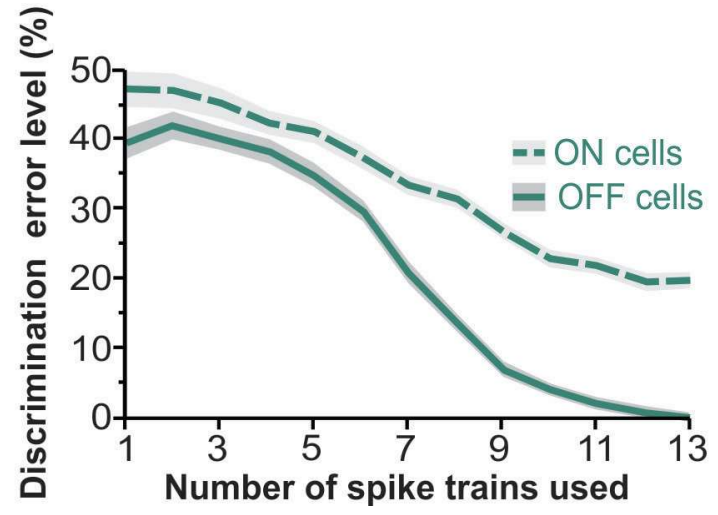

Figure 3:

Small chirps on high frequency beats can be discriminated and are coded by graded responses. A. Example responses of $3 \mathrm{ON}$ and 3 OFF-cells to small chirps on $120 \mathrm{~Hz}$ beats. Similar to big chirps, ON-cells are inhibited, and OFF-cells show varied increases in firing rate. B. Average firing rate for OFF(left) and ON-cells (right) during presentation of two different small chirps. There appears to be greater difference in averaged responses for OFF-cells than ON-cells. C. Discrimination performance based on the response of $\mathrm{ON}$ and OFF cells. OFF-cells can discriminate between varying small chirps on high frequency beats with more accuracy than ON-cells. Shaded area indicates standard error. 
A

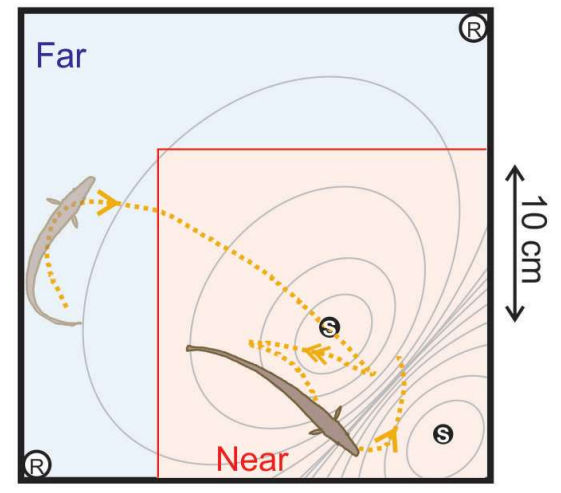

B

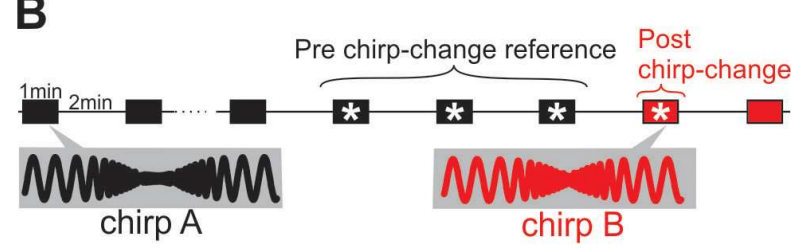

C

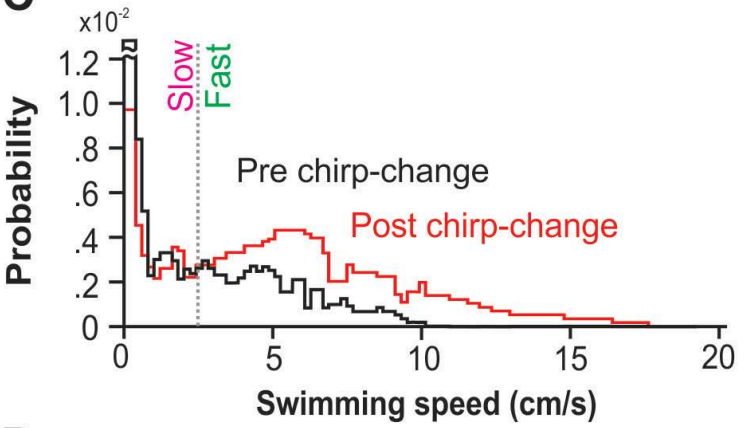

D

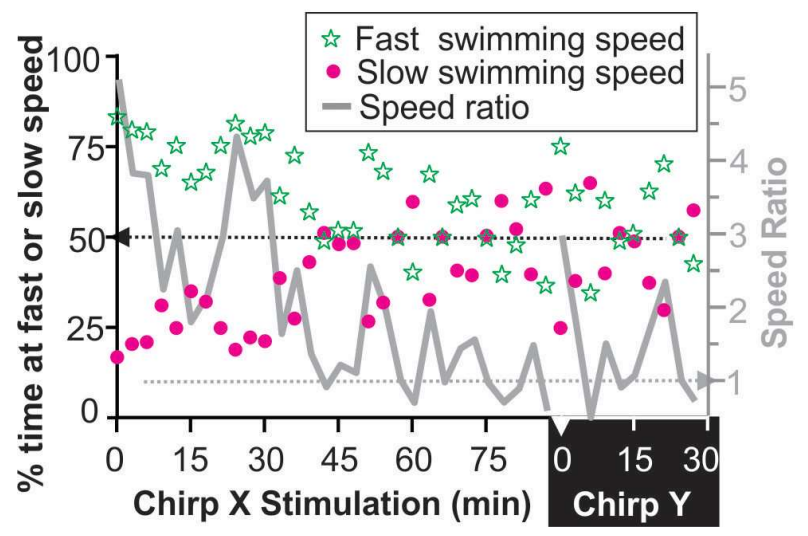

Figure 4:

\section{Experimental design for \\ habituation/dishabituation trials. A.}

Schematic of the experimental tank. Recording electrodes $(\mathrm{R})$ recorded electrical activity as the fish swam freely (example track shown in yellow). Stimulating electrodes (S) were located in one corner of the tank, creating a fish-sized field potential (isopotential field lines illustrated in gray). Two zones, far (blue) and near (red) were defined, splitting the tank on two equal areas relative to the stimulus. B. Chirp stimuli were played $1 \mathrm{~min}$ on $/ 2 \mathrm{~min}$ off for $90 \mathrm{~min}$ (black boxes). After $90 \mathrm{~min}$, a stimulus of the same chirp category but with different properties was played (red boxes). Asterisks mark stimulation bouts used to define pre chirpchange and post chirp-change responses $\mathbf{C}$. Example of swimming speed distribution during the habituated (black) phase of the stimulation and the first stimulus presentation after changing chirp properties (red). The median of the swimming speed in the three stimulation bouts prior to chirp-change (dashed line) is used as a threshold between slow and fast swim speed. D. Speed ratio is calculated by first defining fast and slow swimming speed based on the threshold defined in panel $\mathrm{C}$ of this figure. The proportion of slow swimming (circles) and fast swimming (stars) is determined for each stimulation bout. The speed ratio (fast/slow, corresponding to star/circle) is then calculated (grey line; see y axis on the left). Responses where $50 \%$ of the swim speeds are slow and 50\% are high (points on the black dotted line) lead to speed ratios of one (grey dotted line). The data presented here are noisy since they come from a single trial (the same as used for panel C). Nevertheless, the trend is visible: the ratio is high in the first 30 min and drops to 1 just prior to the change in chirp. The first stimulation bout post chirp-change result in a fairly high speed ratio of 3 . This procedure normalizes the speed measure for each fish and focuses on the time spend in active (fast) vs quiescent (slow) motion rather than absolute speed averages. 


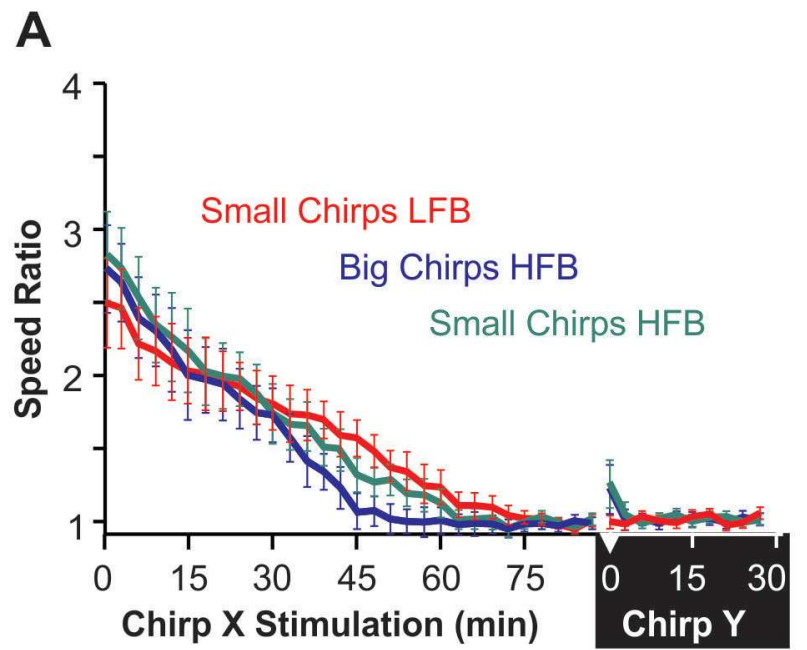

B

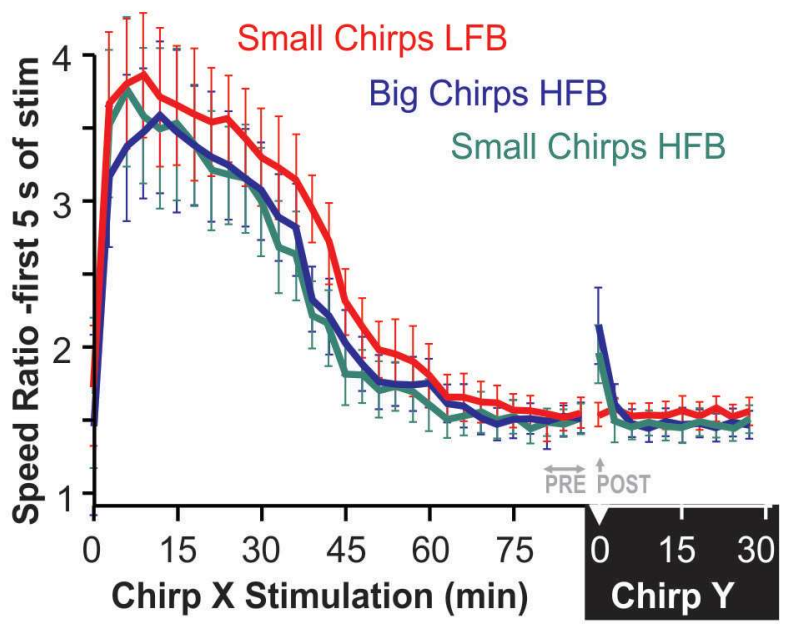

C $\underline{\text { Small Chirp LFB Big Chirp Small Chirp HFB }}$

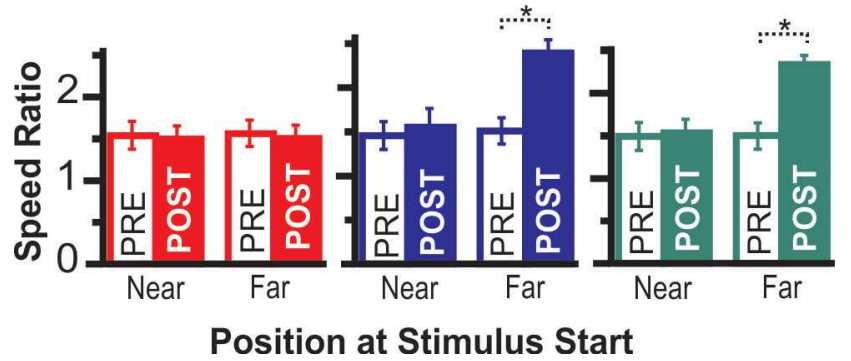

Figure 5:

\section{Both small chirps and big chirps on high frequency beats cause dishabituation. A.} Average speed ratio as a function of stimulation bout. Low frequency beat stimuli (LFB) show no dishabituation, high frequency beat stimuli (HFB) show small, but non-significant effects. B. Average speed ratio during the first 5 seconds of each stimulation bout. Speed increases for novel chirps on high frequency beats indicate dishabituation. C. Fish farther from the stimulating electrodes at start of the novel chirp increase speed only for chirps on high frequency beats. Dotted brackets with asterisks indicate significant differences (average \pm s.e.; paired t-test, $\mathrm{p}<0.01$ ). For each stimulus category, 80 to 85 trials were conducted; in each category $43 \%$ to $56 \%$ had fish classified as far from the dipole just prior to the start of the novel stimulus. 


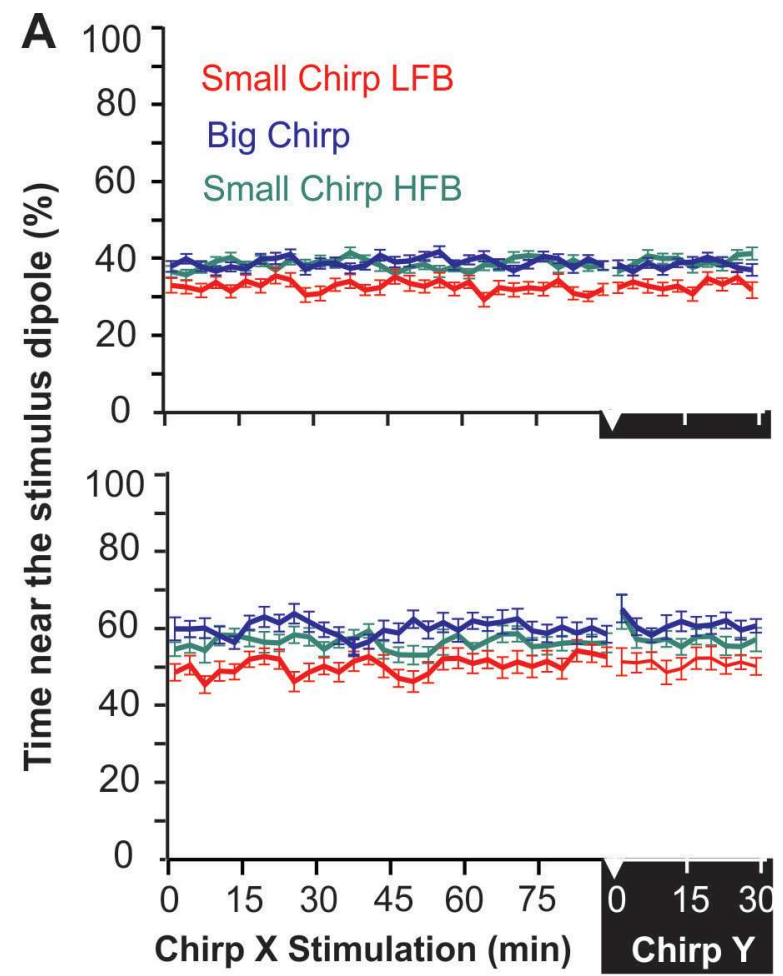

B

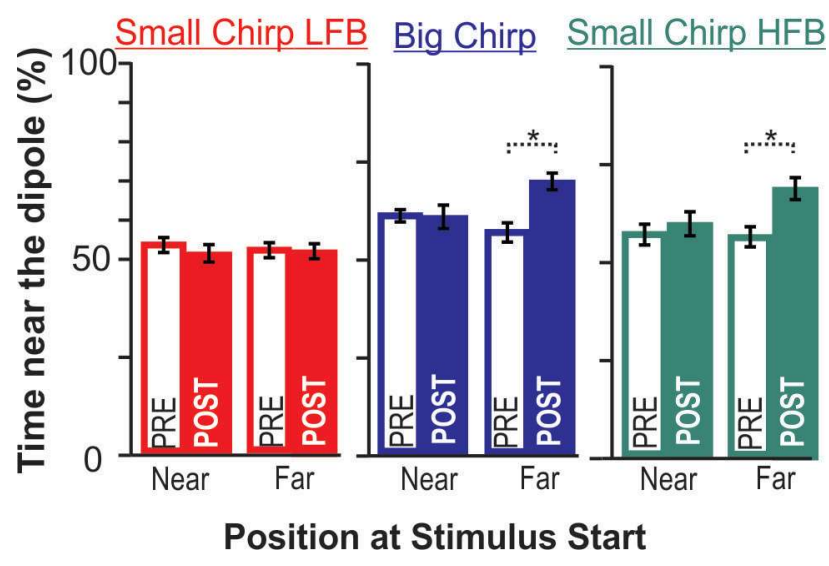

Figure 6:

\section{Fish change location in tank with}

dishabituation. A. Time spent in the area near the stimulation dipole for each bout in the sequence averaged across all fish. Fish typically spent $30 \%$ to $60 \%$ of the time near the stimulus dipole but position did not change with repeated stimulation. This lack of habituation across stimulation bouts is observed whether using the entire recording (top panel) or the first $5 \mathrm{~s}$ (bottom panel). B. Position of the fish during stimulation for stimuli preceding novel chirp (white bars) or following novel chirp (filled bars) for fish far or near the stimulation source just prior to stimulus onset. Using the first five seconds of stimulations, data show that fish farther from stimulating electrodes at the start of the novel chirp moved closer for chirps on high frequency beats. Dotted brackets with asterisks indicate significant differences in the time spent near the stimulus source (average \pm s.e.; paired t-test, $\mathrm{p}<0.01$ ). 


\section{Chapter 3}

Prologue

The previous chapter demonstrates how signals with different structures are encoded via different strategies. It is unknown whether the burst and tonic firing coding strategies observed in A. leptorhynchus are unique to this species as a result of the specific properties of this species signals, or if that strategy is generalizable across all species of apteronotids making it a general principle guiding the matching of sender and receiver via physiology. Bursting and tonic firing for different signal types are common coding strategies across many sensory systems (Krahe \& Gabbiani, 2004; Sherman, 2001) and it would not be surprising if such a pattern was conserved across apteronotids. However, the diversity in signal structure among this group would argue that there might also be diversity in coding strategies to facilitate signal detection.

Comparative studies of the closely related $A$. albifrons show that this species produces chirps much longer than A. leptorhynchus rendering the structure of the signal very different, particularly for the signal type that elicits burst in A. leptorhynchus. Furthermore, A. albifrons generally produce chirps under different social circumstances (Dunlap \& Larkins-Ford, 2003; Kolodziejski, Sanford, \& Smith, 2007). These key differences make this species a good candidate for determining if differences in signal structure and use correlate with differences in encoding when compared to A. leptorhynchus.

In this chapter, I show that $A$. albifrons show distinct differences in chirp coding and neural response properties from A. leptorhynchus. The chirp coding strategy found in these fish appears to be adaptive for the way chirps are used by this species as they do not employ a burst coding strategy, like that seen in A. leptorhynchus. Moreover, the lower coding accuracy for chirps on high frequency beats mirrors the very rare use of chirp in this context. These data 
show evidence of sender-receiver matching reflecting differences in the context of use of the signals.

Note: This chapter is taken directly from my publication:

"Neural Processing of Communication Signals: The Extent of Sender-Receiver Matching Varies across Species of Apteronotus"; Kathryne M. Allen, Gary Marsat, eNeuro Published 4 March 2019, 6 (2) ENEURO.0392-18.2019.

Gary Marsat designed the experiments, Kate Allen designed the experiments, collected and analyzed all data. 


\section{Abstract}

As communication signal properties change, through genetic drift or selective pressure, the sensory systems that receive these signals must also adapt to maintain sensitivity and adaptability in an array of contexts. Shedding light on this process helps us understand how sensory codes are tailored to specific tasks. In a species of weakly electric fish, Apteronotus albifrons, we examined the unique neurophysiological properties that support the encoding of electrosensory communication signals that the animal encounters in social exchanges. We compare our findings to known coding properties of the closely related species, Apteronotus leptorhynchus, to establish how these animals differ in their ability to encode their distinctive communication signals. While there are many similarities between these two species, we found notable differences leading to relatively poor coding of the details of chirp structure occurring on high-frequency background beats. As a result, small differences in chirp properties are poorly resolved by the nervous system. We performed behavioral tests to relate A. albifrons chirp coding strategies to its use of chirps during social encounters. Our results suggest that $A$. albifrons do not exchange frequent chirps in non-breeding condition, particularly when the beat frequency is high. These findings parallel the mediocre chirp coding accuracy in that they both point to a reduced reliance on frequent and rich exchange of information through chirps during these social interactions. Therefore, our study suggests that neural coding strategies in the central nervous system vary across species in a way that parallels the behavioral use of the sensory signals. 


\section{Significance:}

Sender-receiver matching is a phenomenon commonly observed in the peripheral nervous system. It enables communication production and reception to evolve together so that conspecifics remain sensitive to important signals. In this manuscript we examine this phenomenon in the central nervous system of the weakly electric fish $A$. albifrons and compare its processing of communication signals to a closely related species (A. leptorhynchus). Although some differences across the two species can help tailor the system for processing species-specific signals, our data indicate that encoding of communication signals in A. albifrons is not as sensitive as in A. leptorhynchus for certain categories of signals. Our data support the idea that the extent of sender-receiver matching can vary as a function of behavioral needs. 


\section{Introduction}

Sender and receiver matching facilitating communication has been demonstrated across diverse groups, including songbirds (Brumm and Slabbekoorn, 2005), anurans (Schul and Bush, 2002), and insects (Neuhofer et al., 2008). Peripheral tuning and call matching are well characterized, and studies have shown evidence for sender and receiver matching in response to auditory coding of courtship song (Gerhardt and Schwartz, 2001; Tootoonian et al., 2012; Woolley and Moore, 2011) but little work has been done in other sensory modalities. We aim to identify species-specific variations in coding properties of central electrosensory neurons and link them to the divergent use of communication signals, showing that the extent of speciesspecific adaptations results in a variably tight sender-receiver match.

Weakly electric fish are ideal for examining diversification in signal production and reception. Apteronotids share a common mode of communication, modification of their electric organ discharge (EOD), but exhibit huge variety in signal structure. Voluntary modulations of EOD (chirps) vary dramatically in properties such as duration, frequency, and shape, even between closely related species (Turner et al., 2007; Zakon and Smith, 2002). Likewise, chirp reception is influenced by EOD waveform shape (Petzold et al., 2016), chirp features or structure (Benda et al., 2006; Marsat et al., 2012), and social environment (Stamper et al., 2010). The apteronotid electrosensory system could have coding properties generic enough to process these signals efficiently despite differences in structure and use between species; alternatively, differences in chirp processing could reflect adaptations of the electrosensory system to these differences in chirp production. 
Relative EOD frequencies of interacting fish greatly influences chirp perception. These fish perceive ongoing amplitude modulations (AM beat) that are the product of two fish with different EOD frequencies interacting at close range (Bastian, 1981). Chirping modulates this beat (Hagedorn and Heiligenberg, 1985; Zupanc and Maler, 1993). In A. leptorhynchus, low frequency beats are typical of agnostic encounters between fish of similar sex and size, and fish respond with frequent small chirps $(<100 \mathrm{~Hz}$ increase) (Hupé and Lewis, 2008). Higher frequencies occur between fish of opposite sexes or with large differences in body size and more often elicit big chirps (>100Hz), typical of courtship (Hagedorn and Heiligenberg, 1985; Henninger et al., 2018).

Chirp coding has been well characterized in A. leptorhynchus. The two categories of signals described above produce different responses in the primary sensory area, the electrosensory lateral line lobe (ELL). Small chirps on low frequency beats cause stereotyped bursting among ELL pyramidal cells. This encoding strategy, and the structure of the signal itself, means variations in small chirps cannot be discriminated (Allen and Marsat, 2018; Marsat et al., 2009). Conversely, both big and small chirps on high frequency beats produce heterogeneous responses and chirp variations are accurately discriminated (Allen \& Marsat, 2018; Marsat \& Maler, 2010).

The mechanisms of chirp production are similar in A. albifrons and A. leptorhynchus, but chirp structure differs in a number of ways. Chirp duration is the most notable difference in these species. A. leptorhynchus chirps are typically tens of milliseconds long, whereas A. albifrons chirps are generally over 100 milliseconds long (Dunlap et al., 1998; Turner et al., 2007; Zupanc and Maler, 1993). This lengthening is thought to be a recent evolutionary change, with shorter chirps representing the basal state of this branch (Smith et al., 2016). A. albifrons show 
differences in frequency tuning from A. leptorhynchus (Martinez et al., 2016) that may be adaptations for coding these long chirps. Additionally, A. albifrons chirps typically do not fall into discreet "small" and "big" chirp categories like those of A. leptorhynchus (Turner et al., 2007), and chirps of varying durations and frequency are used in all contexts (Kolodziejski et al., 2007). Therefore, it is unknown whether varying chirps have different neural and behavioral impacts in A. albifrons.

In this study, we examine coding of conspecific social signals and their behavioral use to understand if the sensory system is adapted to the specific characteristics of the communication system of A. albifrons, and if so, to what extent. We compare our findings to the behavior and physiology of the closely related A. leptorhynchus to identify specific neurophysiological adaptations that reflect differences in the structure and behavioral use of chirps in these two species. 


\section{Methods}

Animals

All animals were housed according to WVU IACUC standards, protocol 151200009.2. Wild-caught Apteronotus albifrons and Apteronotus leptorhynchus were obtained from commercial fish suppliers and housed in small groups (1-10 fish per tank). Tank conductivity was maintained at 200-500 $\mu$ S. Sex was not confirmed, but adult animals with a wide range of EOD frequencies $(600-1300 \mathrm{~Hz})$ were used for all experiments, indicating that we likely had multiple animals of both sexes.

\section{Electrophysiology}

Surgical techniques were identical to those previously described in (Marsat et al., 2009; Marsat and Maler, 2010). Cells of the LS of the ELL were targeted. A. albifrons brain anatomy is very similar to that of A. leptorhynchus, so major landmark blood vessels described in Maler et al., (1991) and electrode depth served as an adequate guide to locate LS pyramidal cells (see Histology). In vivo recordings were made via metal filled extracellular electrodes (Frank and Becker, 1964) and amplified with an A-M Systems amplifier (Model 1700). Data were recorded (Axon Digidata 1500 and Axoscope software) at a 20kHz sampling rate. ON and OFF cells were identified using known response properties, particularly responses to sinusoidal stimulation and spike triggered average waveforms calculated from responses to $0-60 \mathrm{~Hz}$ noise (Saunders and Bastian, 1984).

\section{Histology}

In $\mathrm{N}=9$ fish, correct electrode placement was confirmed by injection of Dextran Texas Red dye (Thermo Fisher, catalogue \# D1829) at recording site using double barreled electrodes, 
similar to methods described in Krahe et al., (2008). After recording, dye was pressure injected with a PicoPump (WPI, PV820). Animals were then anaesthetized and respirated with a solution of Tricaine-S (.5g/L, Western Chemicals) and perfused with 4\% paraformaldehyde (Electron Microscopy Supply, \# 15712) in 1X PBS. Brains were postfixed overnight, sectioned (150 $\mu \mathrm{m})$, and counterstained with Syto59 nuclear stain (Thermo Fisher, \# S11341), which allowed for clear distinction between ELL segments. In all marked sections, correct placement within the LS was observed.

Stimuli

All stimuli were created in MatLab (Mathworks, Inc.) and sampled at $20 \mathrm{kHz}$. Stimulation was provided by a direct modulation of the amplitude (AM) of a carrier artificial EOD phase locked to the fish's own rather than by mimicking a second EOD. This method (described below) is commonly used in similar experiments (Benda et al., 2005; Krahe et al., 2008; Marsat et al., 2009) and allows precise control over the stimulus AM . Baseline EOD was recorded via electrodes near the head and tail of the fish. Each EOD cycle triggered a sine wave generator (Rigol DG1022A) to generate one cycle of a sine wave matched to the animal's own. This signal was then multiplied using a custom-built signal multiplier (courtesy of the Fortune Laboratory, New Jersey Institute of Technology) by the AM stimulus to create the desired modulation of the electric field. Stimuli were played through a stimulus isolator (A-M Systems, Model 2200) into the experimental tank via either two $30.5 \mathrm{~cm}$ carbon electrodes arranged parallel to the fish's longitudinal axis (global stimulation) or two silver chloridized point electrodes $1 \mathrm{~cm}$ apart from each other positioned near the receptive field on the fish's skin (local stimulation). The stimulus strength was adjusted to provide $\sim 20 \%$ contrast (the difference between the maximum and minimum of amplitude modulation divided by the baseline EOD). See Fig1-3 for further comments on stimulus strength. 
Random amplitude modulation (RAM) stimuli consisted of 30 seconds of random noise filtered using either a low pass $(0-20 \mathrm{~Hz})$, band pass $(40-60 \mathrm{~Hz})$ or broadband $(0-60 \mathrm{~Hz})$ Butterworth filter. Each stimulus was played for three repetitions in both global and local stimulation configurations. Sinusoidal amplitude modulation (SAM) stimuli were 2 second long stimuli modulated at 2, 5, 15,30,60, and $90 \mathrm{~Hz}$ with 2 seconds rest between each frequency, repeated at least three times. Step stimulations were $100 \mathrm{~ms}$ long increases or decreases in amplitude repeated for 30 seconds.

Chirp stimuli were created by using recorded EOD samples (courtesy of Dr. Troy Smith, Electric Fish Signal Library) to create a $1000 \mathrm{~Hz}$ template A. albifrons EOD shape, thereby accounting for the actual EOD shape and resulting AM beat shape. Chirps of varying durations, frequency increases, and frequency rise/fall time (Table 1) were each embedded in this template EOD by decreasing the template EOD period by the inverse of the desired frequency increase for each EOD cycle in the duration of the chirp. For each $1 \mathrm{~Hz}$ of frequency increase, the template amplitude was decreased by .11\%, based on Dunlap at al. (1998). Chirps were added at the rate of 1 chirp per second. To this chirper EOD, a second EOD, either $990 \mathrm{~Hz}$ or $900 \mathrm{~Hz}$, was added to create a combined EOD signal with a beat AM frequency of 10 or $100 \mathrm{~Hz}$. The AM of this stimulus was extracted by rectifying and low-pass filtering the combined EOD signal. The AM signal was delivered during the experiments as described above. Each chirp stimulus was played for at least 30 seconds and up to 60 seconds. Due to time constraints, chirps were only played in the global configuration.

\section{Data Analysis}

For all analyses spike trains were first binarized into a sequence of 1's (spike) and 0's (no spike) using a bin width of $0.5 \mathrm{~ms}$. All analyses described here were performed on these binary 
sequences in MatLab. Statistical analyses (ANOVA, Student's t-Test, Wilcoxon rank sum) were performed using the MatLab's statistical analysis toolbox.

\section{Synchronization Coefficient}

The strength of phase locking to SAM stimulation was calculated as

$$
s=\frac{\sqrt{\left(\sum x_{i}\right)^{2}+\left(\sum y_{i}\right)^{2}}}{n}
$$

where $n$ is the number of spikes in the analysis, and $x$ and $y$ are the cosine and sine of the stimulus' phase at which spike $i$ occurs (Goldberg and Brown, 1969; Marsat and Pollack, 2004). The vector strength, $s$, ranges from 0 to 1 , with 1 being perfect precision in responding to a given phase of the cycle.

\section{Coherence}

Lower bound (stimulus-response) coherence is a measure of linear coding of the stimulus and is calculated by comparing the spike train against the RAM stimulus (Borst and Theunissen, 1999). Stimulus-response ( $\mathrm{S}$ and $\mathrm{R}$ respectively) magnitude-squared coherence $\left(C_{S R}\right)$ was calculated as a function of frequency $(f)$ according to:

$$
C_{S R}(f)=\frac{\left|P_{S R}(f)\right|^{2}}{P_{S S}(f) P_{R R}(f)}
$$

where $P$ indicates the power-spectral or cross-spectral densities.

Upper bound (response-response) coherence measures the total information potentially coded in the response, including linear and non-linear information. It is measured by comparing 
multiple responses of one neuron to each other to determine response reliability (Borst and Theunissen, 1999). Coherence between responses $R i$ and $R j$ was calculated as:

$$
C_{R i R j}(f)=\sqrt{\frac{P_{R i R j}(f) P_{R j R i}(f)}{P_{R i R i}(f) P_{R j R j}(f)}}
$$

and averaged across all pairwise combinations of responses.

Envelope responses were determined by computing the lower-bound coherence between the responses and the envelope of the $40-60 \mathrm{~Hz}$ RAM stimuli. To do so, the stimulus $S(t)$ in the lower-bound analysis described above was replaced by the envelope $E(t)$ calculated by rectifying and low pass filtering the $40-60 \mathrm{~Hz}$ noise stimulus AM. Similar results were obtained when using a Hilbert transform to calculate the envelope signal.

\section{Burst detection}

Bursts in RAM and chirp responses were identified by creating a histogram of all interspike intervals (ISIs) in the response. Bursting neurons have a bimodal, non-Poisson distribution of ISIs, allowing us to visually identify a threshold ISI at the upper boundary of intra-burst intervals. Spikes in groups with ISIs below the threshold were classed as occurring in bursts, while all remaining spikes were classed as tonic. This method is similar to that described in (Ávila-Åkerberg et al., 2010)

\section{Chirp detection and discrimination analysis}

Analysis of chirps for detection and discrimination is based on Allen and Marsat (2018). Our analysis accounts for both the firing rate as well as the temporal pattern of spikes to quantify how similar or dissimilar spiking patterns are (van Rossum, 2001). For detection analysis, a 
window around the chirp $\left(\mathrm{R}_{\mathrm{c}}(\mathrm{t})\right)$ of length $L(50,105$, or $205 \mathrm{~ms})$ was extracted from the filtered spike train and compared to a window of beat of the same size $\left(R_{b}(t)\right)$. Different window sizes led to qualitatively similar results, and those using $205 \mathrm{~ms}$ are shown. The responses were

convolved with an $\alpha$ filter, $f(t)=t * \exp \left(-\frac{2.45 t}{\tau}\right)$, with $\tau$ being the width of the function at half maximum $(3,10,30$, and $100 \mathrm{~ms} ; 30 \mathrm{~ms}$ shown) (Machens et al., 2003). Detection accuracy is calculated for population responses by averaging multiple spike trains:

$$
P R_{i}(t)=\frac{\sum_{i=t}^{n} R_{i}(t)}{n}
$$

The result $\left(P R_{i}(t)\right)$ represents a population of neurons presented with the same stimulus and mimics a neuron integrating postsynaptic potentials with similar weights. Distance $(D)$ was calculated for all sets of combined responses, $P R_{c}(t)$ and $P R_{b}(t)$, creating an array of response distances for each comparison using the function:.

$$
D_{c b}=\frac{\sum_{t=o}^{L}\left[R_{c}(t)-R_{b}(t)\right]^{2}}{L}
$$

The probability distributions of the values in these arrays $\left(P\left(D_{c b}\right)\right.$ or $\left.P\left(D_{b b}\right)\right)$ were used for analysis. Receiver operator characteristic curves were calculated by varying a threshold distance $(T)$ to separate chirp and beat responses. For each threshold the probability of detection $(P D)$ was calculated as the sum of $\left(P\left(D_{c b}>T\right)\right)$, and the probability of false alarm $(P F)$ as the sum of $\left(P\left(D_{b b}<T\right)\right)$. The error level for each threshold value is $E=P F / 2+(1-P D) / 2$. The values shown in Figure 3 are the minimum calculated values of $E$ as increasing numbers of spike trains are included in the calculation.

Discrimination analyses were similar to those for detection, but rather than comparing chirp and beat responses, the distances between two different chirps were compared. Similar to 
detection analysis, windows of chirp responses $(R(t))$ of length $L(50,105$, and $205 \mathrm{~ms})$ were used for analysis and the results using $205 \mathrm{~ms}$ are shown. The responses were convolved with an $\alpha$ filter and population averaged as described above. Up to 200 random combinations of spike trains from all recorded neurons were used for all comparisons as more become computationally prohibitive without improving results. Responses to a given chirp against a different chirp (x vs. y) were compared as well as multiple responses to the same chirp (x vs. x). Spike train metric distances $\left(D_{x y}\right.$ or $\left.D_{x x}\right)$ the ensuing distribution and error rates were calculated as described for the detection analysis.

Adaptation

Adaptation to stimuli was measured by using the MatLab Curve fitting toolbox to fit an exponential curve to a plot of instantaneous firing rate for each neuron resulting in a time constant $\tau$. The portion of the response to use for fitting was determined by selecting the time of peak firing rate and the following 500ms.

\section{Behavior}

Twenty-eight behavior trials were conducted in a small tank $(27 \times 27 \times 14 \mathrm{~cm})$ containing water with conductivity, $\mathrm{pH}$, and temperature matched to the home system, and one shelter tube. The tank was enclosed to block ambient light, lit with infrared lights and all trials were recorded via infrared camera (Logitech HD Pro Webcam C920). 14cm carbon rod electrodes placed diagonally from each other in each corner of the tank recorded electrical activity, which was then amplified (A-M Systems, Model 1700) and recorded using a computer sound card.

Stranger fish from different home tanks were paired. Defining physical features (size, markings) and EODf were noted to avoid repeatedly testing the same pairing. Individual fish 
were used no more than three times, with at least seven days between each trial. One fish was selected and allowed to acclimate to the test tank for 20 minutes before the introduction of the second fish. Recording began immediately upon introduction of the intruder fish. Interactions were recorded for five minutes.

To detect chirps, we used a custom MatLab script to create a spectrogram of the electrical recordings to identify individuals and mark chirp times. Chirps were visually identified as $>10 \mathrm{~Hz}$ abrupt frequency increases. 


\section{Results}

\section{Diversity in conspecific chirp responses}

In multiple gymnotid species the lateral segment (LS) of the ELL serves as the primary location for encoding of communication and social signals (Marsat et al., 2009; Metzner, 1999; Metzner and Juranek, 1997), thus we targeted our recordings to pyramidal cells in that segment. To characterize the pattern of responses of $A$. albifrons pyramidal cells we played a series of chirps mimicking the natural range of reported A. albifrons chirps (Turner et al., 2007). Additionally, for comparison we used a small number of chirps with properties more typical of A. leptorhynchus (Dunlap et al., 1998; Zupanc and Maler, 1993). Detailed descriptions of chirp properties used are located in Table 1. In addition to playing chirps with diverse properties, we also varied the frequency of the beat, presenting chirps on both low $(10 \mathrm{~Hz})$ and high $(100 \mathrm{~Hz})$ frequency signals.

Figure 1 displays a representative selection of the diversity seen in chirp responses for both ON-type and OFF-type pyramidal cells. Qualitatively, responses appear to be heterogeneous with variability between inhibition, excitation, and desynchronization. On high frequency beats (Fig 1A) we see a transient increase in tonic firing rate in OFF-cell while ONcells are briefly and weakly inhibited during the chirp. On low frequency beats (Fig 1B), the responses also consisted of brief increases in firing rates, but were additionally characterized by periods of inhibition lasting the entire duration of the chirp. We did not observe synchronized burst firing across the population that is typical of $A$. leptorhynchus' responses to small chirps on low frequency beats (Marsat et al., 2009; Marsat and Maler, 2010), the strategy that limits ability to discriminate these chirps (Allen and Marsat, 2018). Therefore, all chirps appear to be coded primarily through increases in firing among OFF-cells, and inhibition and desynchronization and cancelation of the beat (See Figure 1-1) of ON-cells. These results suggest that A. albifrons 
pyramidal cells uses the same coding patterns for all chirp types, but possibly exhibit differences based on beat frequency. Furthermore, a strong adaptation (see Figure 1-2) in firing rate seen between the beginning and end of the chirp response could cause chirps of different length to be poorly discriminated. Therefore, we next quantified how well the neural responses could support both detection and discrimination of chirps.

\section{Detection of chirps varies with beat frequency}

We used a spike metric distance to measure the variability in firing patterns and how reliably an ideal observer could distinguish chirp responses from beat responses. The results are presented as an error level as a function of the number of neural inputs pooled in the analysis. More efficient encoding would lead this measure to drop faster from 0.5 (chance level) as a larger population of neurons is used in the detection task.

A small number of ON-cells (about eight) are sufficient to accurately detect chirps, comparable to A. leptorhynchus (Marsat and Maler, 2010) (Fig 2A). However, this level of accuracy only holds for chirps on the $10 \mathrm{~Hz}$ beat. ON-cell performance on $100 \mathrm{~Hz}$ is extremely poor, even with high numbers of spike trains included in the analysis (Fig 2B). OFF-cells are also able to detect chirp occurrence on low frequency beats for all chirp types (Fig 2C) but on $100 \mathrm{~Hz}$ detecting chirps was less reliable and accurate (Fig 2D). The data suggest that with a large enough sample of neurons accurate detection is possible, but less robustly than on low frequencies. Therefore, while chirps in all contexts are potentially detected, detection sensitivity is much higher in low frequency contexts.

\section{Discrimination between chirps also varies}

We assessed the amount of information carried by the response pattern about chirp properties that could support the discrimination of chirp variants. This analysis is similar to that 
used for chirp detection, but rather than comparing responses of chirps to beats, we compare responses between chirps. While A. albifrons chirps are not typically grouped into distinct categories, we still use the terminology used in A. lepthorhynchus to describe the two ends of the spectrum of chirp properties (small $<150 \mathrm{~Hz}$ increase; big $>150 \mathrm{~Hz}$ ). Figure 3 shows discrimination ability for chirps grouped by size (See Table 1; Small chirps: 5, 6, 7, 8; Big chirps: 1, 2, 3, 4, 9, 10) for both ON and OFF-cells on high and low beat frequencies.

Chirps of all types are more easily discriminable when presented on $10 \mathrm{~Hz}$ beats (Fig $3 \mathrm{~A}$ ) rather than on $100 \mathrm{~Hz}$ beats (Fig 3B). Performance for chirps on high frequency beats was variable: accurate discrimination was achieved only for chirps with large differences in properties (Fig 3-1) and both ON and OFF cell responses allow qualitatively similar discrimination accuracy. These results are unexpected, considering that the same analysis in $A$. leptorhynchus shows that chirps on high frequency beats can be discriminated well using as few as six neurons, and conversely exhibit poorer discrimination on low frequencies (Marsat and Maler, 2010) where A. albifrons performs best. Furthermore, we do not observe an asymmetry in coding accuracy between ON and OFF cells as observed in A. leptorhynchus.

When looking at which chirps are well discriminated, chirp duration and the steepness of the frequency rise seem to be the most discriminable features, while total frequency increase and number of peaks are less influential on coding (Fig 3-1). However, discriminating the most easily separated chirps is prone to error and requires a larger pool of neurons on high frequency beats. These data indicate that while qualitative observations would suggest that chirp coding is similar between A. albifrons and A. leptorhynchus, there are quantitative differences in the information content about chirps within the electrosensory system. To determine which aspects of the neural 
responses could be responsible for changes in chirp coding, we characterized a wide variety of response properties using an assortment of stimulation protocols.

\section{Frequency tuning and neural coding}

Encoding amplitude modulations (AM) in stimuli is crucial for the perception of social signals. As shown above, A. albifrons neurons respond variably to chirps, which consist of both high frequency AM signals and the low frequency changes in the contrast of the AM (i.e. the envelope). We asked whether the accuracy of detection and discrimination of chirps by $A$. albifrons ELL neurons reflects species-specific frequency tuning properties.

Communication signals cause spatially diffuse stimulation as compared to the localized electric fields of prey items. We simulate this difference by altering the geometry of our stimulating dipoles. Using a large dipole (see methods) that simulates diffuse communication signals drives both the feedforward responses from electroreceptor to pyramidal neuron and feedback pathways driven by broad receptive fields. To separate the effects of feedback from the tuning of the feedforward circuit we also stimulated only the receptive field of each cell with a small local dipole simulating localized objects or prey (Bastian, 1986; Chacron et al., 2005).

In response to simple sine waves, $A$. albifrons exhibit the highest degree of coding fidelity at low frequencies. Firing rate for each sine cycle peaks at $5 \mathrm{~Hz}$ for all cell types and stimulation configurations (Fig 4A). Maximum phase locking also peaks at low frequencies (Fig 4B). These values closely match previously reported values (Martinez et al., 2016). Temporal coding accuracy is conveniently quantified using random noise stimuli and information theory (Borst and Theunissen, 1999). Lower-bound coherence reflects the amount of information encoded linearly whereas upper bound coherence takes in account both linear and non-linear 
aspects of the response (Fig 4C). Both ON and OFF cell coherence curves peak at similar frequencies in the 5-20 Hz range (Wilcox Rank Sum, p=.19). However, OFF-cells are clearly low-pass, and exhibit a sharp decline in coherence to higher frequencies, while ON-cells exhibit a slightly broader lower-bound coherence (see Fig 5 and accompanying text for more details).

The movement of fish as well as the amplitude changes caused by chirps create low frequency changes in AM contrast, or envelopes (Stamper et al., 2013). During chirps, the AM will be high frequency (tens to hundreds of hertz above the beat frequency) whereas the envelope of the chirp is low frequency (e.g. 100ms chirp will lead to a $\sim 10 \mathrm{~Hz}$ envelope). Therefore, the coding of chirps' low frequency content must be investigated using envelope stimuli. We characterized envelope coding using stimuli consisting of RAMs with AM frequencies between $40 \mathrm{~Hz}$ and $60 \mathrm{~Hz}$, containing envelope frequencies of $0-20 \mathrm{~Hz}$ (Middleton et al., 2006). Although pyramidal cells do encode the envelope of these stimuli (Fig 4D), the coding accuracy is low compared with the envelope coding observed in A. leptorhynchus (Chacron, 2006; Middleton et al., 2006; Fig 5-1). OFF-cells are particularly relevant for chirp discrimination. This is because chirps consist of decreases in envelope amplitude, which are best encoded by OFF-cells (Marsat and Maler, 2010). The observed poor coherence to envelope stimuli, particularly for OFF-cells in receiving global stimulation, may in part cause the poor chirp discrimination we observe.

\section{Burst Firing and Chirp Detection}

As described in Marsat et al. (2009) burst firing serves an important role in $A$. leptorhynchus for the efficient detection of extremely short chirps. As qualitatively shown above in Figure 1, no conspecific chirps reliably produced a burst response in A. albifrons. This implies that $A$. albifrons may not be using this dedicated code for detection of very brief chirp signals. In A. leptorhynchus the coding of small chirps is directly related to beat frequency. On low 
frequencies, small chirps are shorter than the period of one beat cycle. As beat frequency increases and the period becomes shorter, small chirps begin to last longer than one cycle. This change in duration relative to beat period mediates a change in how small chirps are coded (Walz et al., 2014). In A. albifrons all chirps are much longer than A. leptorhynchus small chirps, spanning more than one beat cycle even on low frequencies, which may eliminate the need for a feature detection code specifically for extremely short chirps. Thus, the observed lack of bursting may result from the change either in signal structure (duration relative to beat cycle) or from underlying changes to physiology and bursting capability of LS neurons.

To determine if this change in signal coding is a result of signal structure or underlying physiology, we stimulated A. albifrons fish with A. leptorhynchus chirps known to elicit a burst response in $73 \%$ of A. leptorhynchus LS ON-cells (Marsat et al., 2009). We determined burst threshold ISI for each neuron by plotting ISI distribution of chirp and beat responses and manually selecting a threshold that best distinguished between the two (Martinez-Conde et al., 2002) in order to maximize our measure of chirp-specific bursting. The mean burst threshold was $8.0 \mathrm{~ms} \pm 0.7 \mathrm{~ms}$ (s.e.), comparable to the burst thresholds determined for our RAM analyses and in other reports (Ávila-Åkerberg et al., 2010; Chacron and Bastian, 2008; Marsat et al., 2009).The majority of neurons increased firing rate only slightly more in response to A. leptorhynchus chirps than to the beat. There was, however, a small percentage $(\sim 20 \%)$ of ON-cells that did reliably burst more in response to A. leptorhynchus small chirps than to the beat (Fig 5A, C). Most importantly, even the bursty neurons did not respond to the shortest $A$. albifrons chirps with bursts (Fig 5B, D). Unlike A. leptorhynchus small chirps, which may cause either amplitude increases or decreases, depending on chirp time in relation to beat phase, A. albifrons chirps are all long enough to span more than one beat cycle. This chirp length in relation to the beat period 
means that the AM waveform caused by chirps is less variable and the response they elicit is largely independent of the phase at which chirps start. This may mean that having a chirpinvariant coding mechanism, such as bursting, is not necessary in this species.

The low proportion of neurons that burst in response to A. leptorhynchus chirps demonstrates that there are physiologic differences between these two species in regards to burst coding of communication signals. We examined the differences between bursty and non-bursty neurons in more detail by analyzing separately their response to RAM stimuli. The subset of bursty neurons have broader AM tuning (Fig 5C) as tested by comparing the ratio of coherences summed over the $0-30 \mathrm{~Hz}$ vs $30-60 \mathrm{~Hz}$ (bursty cells mean ratio \pm s.d.: $0.91 \pm 0.12$; non-bursty cells mean ratio: $2.35 \pm 1.72$; t-test not assuming equal variance, $\mathrm{p}=0.0047$; similar results were found using the upper-bound coherence). While most ON-cells exhibited peak coherence at frequencies between $10-20 \mathrm{~Hz}$ (non-bursty cells mean peak frequency \pm s.d.: $19.6 \pm 18$ ), these cells had significantly higher peak frequencies as high as $40-50 \mathrm{~Hz}($ mean \pm s.d.: $40 \pm 13.9 \mathrm{~Hz}$; ttest, $\mathrm{p}=0.023$ ) and exhibited high coherence across the range of stimulus frequencies. The remainder of the ON cells displayed the low-pass tuning described in Martinez et al. (2016). These data show that while a subset of ELL neurons are capable of bursting in response to short chirp stimuli, they make up a small percentage of the overall ELL population and bursting does not seem to play a role in conspecific chirp coding.

\section{Feature Detection}

Burst firing may not be a significant aspect of communication coding in A. albifrons, but other uses for burst coding may be conserved. During spontaneous activity, we observed a baseline firing rate of $13.54 \mathrm{~Hz}( \pm 1.37$ s.e. $)$ with $17.01 \%$ ( \pm 2.55 s.e. $)$ of spikes in bursts. Thus, the pyramidal neurons of $A$. albifrons are physiologically capable of bursting. In $A$. 
leptorhynchus burst firing can reliably signal the presence of spatially localized prey-like stimuli (Gabbiani et al., 1996; Oswald et al., 2004). We examined bursting in response to local RAM stimulation (Fig 6A) to determine if bursting could serve similar prey detection functions in $A$. albifrons. The ISI histogram of the responses clearly showed that the neurons burst to these stimuli (Fig 6B). The proportion of spikes occurring in bursts was as high as $63.07 \%$ ( \pm 5.64 s.e.) for stimuli in the local configuration (Fig 6C). The average stimulus waveform triggering burst vs single spikes follows the pattern observed in other species: slower AM for bursts than for single spikes (Fig 6D). However, in response to RAM stimuli, unlike A. leptorhynchus there was not a large difference in coding error between bursting and tonic spiking (ANOVA, $\mathrm{p}=.10$ ) (Fig 6E). These data suggest that while bursts encode low frequency stimuli in the LS of A. albifrons, they do not appear to implement a burst-based feature detection code for either prey-like stimuli or chirps.

\section{Chirp production and behavior}

Our physiology data suggest that $A$. albifrons are able to detect and discriminate chirps on low frequency beats much more sensitively than on high frequency beats. To test this hypothesis, we recorded electrical behavior from pairs of freely swimming and interacting fish. Chirping behavior did occur but differed from that of A. leptorhynchus in a number of ways. Primarily, overall rates of chirping are dramatically lower than numbers reported from similar studies in A. leptorhynchus (Hupé and Lewis, 2008; Zupanc et al., 2006). Over 28 trials, we recorded a sum of 133 chirps, with a mean of 4.75 ( \pm 0.61 s.e.) chirps per 5 -minute trial whereas in a similar context male A. leptorhynchus produced approximately of 125 chirps per 5 minute trials (Hupé and Lewis, 2008). The maximum number of chirps observed in one trial was 13. Due to the small number of observed chirps, we did not separate them into multiple categories for analysis, although chirps of varying frequency increases and duration occurred. Chirping 
frequency is correlated with the difference in EODf of interacting fish. Smaller differences in EODf correspond to higher numbers of chirps (Fig 7A). However, under our conditions chirping does not appear to be sexually dimorphic. Animals used in behavior experiments were not killed to determine sex, but we grouped them by EODf into high $(>1100 \mathrm{~Hz})$ and low $(<1100 \mathrm{~Hz})$ frequency groups which can correspond to females and males respectively in many populations of A. albifrons (Zakon and Dunlap, 1999). We observed no differences in chirp production between high or low frequency groups (Fig 7B). Further, chirp rate does not vary by pairing type, nor by relative EODf of individuals within each pair under our conditions (Fig 7B). These results confirm previous findings that chirp frequency in this species is not sexually dimorphic (Dunlap et al., 1998).

Chirp timing plays an important role in mediating A. leptorhynchus interactions as they frequently exchange chirps in an echo response pattern (Henninger et al., 2018; Hupé and Lewis, 2008; Zupanc et al., 2006), a behavior where one fish responds to the chirp of another fish by chirping back within $<1 \mathrm{~s}$. To determine if chirp timing plays a similar role in A. albifrons interactions, we analyzed inter-chirp intervals for all trials in which both fish chirped. The resulting distribution of all chirp latencies between fish indicates inter-chirp latencies much longer than are typical of A. leptorhynchus echo responses (Fig 7C). Of all recorded chirps, only two occurred within $2.5 \mathrm{~s}$ of each other, making an echo response unlikely to be an important feature of A. albifrons interactions. Furthermore, inter-chirp intervals for a given fish follow a Poisson distribution (7D) indicating that chirp production is not produced in stereotyped sequences. The lack of echoing, the long inter-chirp intervals, and the low rate of chirping in general, suggest that $A$. albifrons do not rely on the detailed decoding of individual chirp characteristics as is the case in A. leptorhynchus. 


\section{Discussion}

We found that neurons in A. albifrons' primary sensory area are able to detect chirp occurrence and do encode chirp identities on low frequency beats. However, they discriminate between chirps less accurately when chirps are presented on high frequency beats. Rather than producing discrete burst responses for small chirps and tonic responses for big chirps, all chirps in this species are encoded via graded and heterogeneous increase or decreases in firing rate. We found several adaptations in the basic response properties that may explain the neural responses to conspecific chirps. These adaptations, frequency tuning in particular, may facilitate encoding long chirps in A. albifrons as well as enhancing chirp coding in the behavioral context in which chirps are used most often. Therefore, we also asked whether chirping behavior could explain differences in chirp coding efficiency for high and low frequency beats. We found that the pattern of chirp use during common social interactions is very different from that of closely related species: $A$. albifrons produces very few chirps and do not respond to each other with echo chirps. Infrequent chirping is particularly pronounced in the context of high-frequency beats suggesting that certain types of interactions are not mediated by chirps. We suggest that the observed differences in chirp coding accuracy on high frequency beats may reflect a relaxation in demands for exchanging information via chirps in this context.

\section{Low frequency tuning could be adaptive for chirp coding}

Our results confirm that pyramidal cells in the LS of $A$. albifrons are low pass. This finding parallels the low frequency envelopes produced by long $A$. albifrons chirps. However, the impact of this AM tuning on chirp coding depends on the way AMs and envelopes are processed in this system. Whereas the envelopes of chirps are low frequency, the AM are high frequency. Therefore, low frequency tuning to AM is not automatically helpful. We found that 
the coding of low-frequency envelopes was relatively poor, particularly in OFF cells, which -in A. leptorhynchus- are best at coding chirp envelopes. However, if envelope response is synthesized before the pyramidal cells (e.g. at the electroreceptors as could be the case in $A$. leptorhynchus (Savard et al., 2011)) low frequency tuning to AM could benefit chirp coding since the input to the pyramidal cells would contain low-frequencies (Metzen et al., 2018). Nevertheless, we show that the neural response does not accurately reflect the chirp envelope and that envelope coding of decreases in amplitude of RAM envelope stimuli is poor. Our data replicate the findings of Martinez et al. (2016) that also show poor coding of envelope stimuli, with coherence values of approximately 0.1 . It is possible that without low frequency tuning, chirp and envelope coding would be even worse. Thus, low frequency tuning may compensate for other properties that hinder envelope coding (e.g. amount of rectification performed by the receptors; (Savard et al., 2011)).

\section{The role of burst coding}

Bursts also often play an important role in coding specific features of communication signals (Creutzig et al., 2009; Fujimoto et al., 2011) as it is the case for A. leptorhynchus where bursting enhances detectability of small chirps on low frequency beats (Marsat et al., 2009). $A$. leptorhynchus small chirps are unusual in that, for low frequency beats, they span less than a full cycle of the beat. Consequently, the same chirp can be perceived as a sharp decrease in amplitude, a sharp increase, or a mix of the two, depending on the phase of the beat at which the chirp starts (Benda et al., 2005). It may be because of this aspect of chirp structure and its shortness, that the sensory system of A. leptorhynchus uses bursts to enhance detectability rather that optimizing the coding of these chirps' properties. A. albifrons is not subject to the same constraints. The perceived AM of a chirp is largely independent of beat phase since chirps span several cycles of the beat and their long duration might make them more conspicuous (Petzold et 
al., 2016). Using noise stimuli, we showed that burst coding in the LS is different in A. albifrons. Since bursts are not used to encode chirps, and because LS primarily focuses on processing communication signals, it is possible that these neurons do not need to use burst as a feature detection signaling mechanism as in other species (Gabbiani et al., 1996; Oswald et al., 2004).

\section{Chirp coding and behavior}

Our chirp response analysis shows that all chirps are coded with graded increases and decreases in firing rate containing some information about chirp properties but, for high frequency beats, coding accuracy is inefficient at supporting both chirp detection and discrimination. Our behavioral data might shed some light on this apparent inefficiency at coding chirps across all social contexts. We show that, in our conditions, A. albifrons chirp relatively infrequently to meditate dyad interactions. Furthermore, we show that there is a tendency to chirp less when the beat frequency is higher. This is supported by previous findings showing that chirp production is more frequent in interactions involving low frequency beats (Kolodziejski et al., 2007) reiterating the relevance of chirp coding on low-frequency beats rather than high frequency interactions.

\section{Comparison to A. leptorhynchus and other electric fish}

Our physiology and behavioral findings are markedly different than those observed in the closely related A. leptorhynchus. Primarily, our data show A. albifrons exhibit poor chirp coding on high beat frequencies, and efficient coding on low beat frequencies. This is directly in contrast with A. leptorhynchus that encode chirps on high frequency stimuli well and exhibit poorer coding of chirp identity on low frequency beats (Allen and Marsat, 2018; Marsat and Maler, 2010). Furthermore, we find that A. albifrons do not rely on the same coding strategy as used in A. leptorhynchus since feature detection through bursts is not involved in chirp coding. Our data clearly demonstrate that despite being so closely related (Smith et al., 2016) the sensory 
coding properties of the neurons encoding communication signals have diverged between $A$. leptorhynchus and A. albifrons. Chirps have been suggested to be short in the ancestors basal to the two species (Smith et al., 2016) and longer chirps a development of the A. albifrons branch. Therefore, it is possible that the differences in frequency tuning we observed in A. albifrons may be adaptive for coding these long chirps.

Our behavioral results suggest that $A$. albifrons also use chirp exchanges very differently than A. leptorhynchus. Unlike the complex chirp interactions observed in A. leptorhynchus, where chirps are omnipresent and play a central role in various type of interactions (Hagedorn and Heiligenberg, 1985; Henninger et al., 2018; Hupé, 2012) A. albifrons chirp infrequently and generally only in low frequency difference interactions (Kolodziejski et al., 2007). While $A$. leptorhynchus have dedicated uses for different chirp types (Henninger et al., 2018), A. albifrons use all types of chirps interchangeably for low beat frequency interactions and may not possess distinct chirp categories (Dunlap et al., 1998; Kolodziejski et al., 2007; Turner et al., 2007). The link between chirp properties and behavior in A. albifrons is also less clear than in $A$. leptorhynchus (Dunlap and Larkins-Ford, 2003). Therefore, we suggest that rather than having distinct coding strategies for different chirp types, they have adopted context specific coding to better encode chirps on low frequency beats where chirps are most likely to be produced.

Chirping behavior has been investigated across many species of wave-type gymnotiforms (Hagedorn and Heiligenberg, 1985; Petzold et al., 2018, 2016; Turner et al., 2007). Details of chirp encoding, however, have only been investigated in one other species, Eigenmannia viscerens (Metzner and Heiligenberg, 1991; Metzner and Viete, 1996; Stöckl et al., 2014). In this species, similarly to A. leptorhynchus, different chirp types are used to mediate agonistic and courtship encounters (Hagedorn and Heiligenberg, 1985). In this species, unlike the apteronotids, 
chirp coding appears to be unaffected by beat frequency, at least at the level of electroreceptors (Stöckl et al., 2014). However, the coding strategy observed in the ELL pyramidal cells is that the duration of the chirp is the primary feature encoded. This feature is coded via brief excitation, then inhibition of both ON and OFF-cells (dependent on sign of the DC component of the chirp) that signals the duration of the chirp (Metzner and Heiligenberg, 1991). This strategy is similar to the coding observed in A. albifrons in low frequency interactions, suggesting that perhaps $A$. leptorhynchus represent the outlier group, and burst detection of chirps is a specialization for their small chirps.

\section{Conditions affecting chirp coding}

Our study did not test for the effects of neuromodulation on neural tuning and chirp coding. Neuromodulation can change cell response properties for different behavioral states (Harris-Warrick and Marder, 1991). Previous work in A. leptorhynchus shows that serotonin enhances pyramidal cell excitability and responsiveness to small chirps on low frequency beats (Deemyad et al., 2013). While we worked on adult animals, we did not determine sex or breeding status, both of which have large effects of the frequency and quality of chirps produced (Smith, 2013). It is likely that the effects of neuromodulation due to behavioral state could affect the reception and encoding of these chirps as well, altering sensitivity to chirps and possibly even coding accuracy in response to behavioral need. This may particularly influence the coding of chirps on high frequency beats, which we observed was surprisingly poor. This kind of interaction is more likely to occur in breeding contexts, so it is possible that animals in breeding condition could be better able to detect and discriminate these signals than the results we show here. We also did not investigate other environmental factors that could influence the sensory specializations of both species, such as microhabitats, prey capture, and general sociality, all of which could drive some of the adaptations seen. 
Our analysis technique is widely used to quantify the encoding performance of sensory neurons (Itatani and Klump, 2014; Mouterde et al., 2017; Neuhofer and Ronacher, 2012), and is biologically realistic (Larson et al., 2009). Nevertheless, other analysis technics could be devised to improve the discrimination accuracy estimate. Weighting the contribution of the different neurons, or weighting them differently across time could be envisioned (Larson et al., 2010). Keeping the different neurons as separate dimensions in the analysis (Houghton and Sen, 2008) or combining ON and OFF-cell responses (Aumentado-Armstrong et al., 2015) could also improve discrimination, as could accounting for neural correlation in response variability (Hofmann and Chacron, 2017). Finally, various more complex decoding methods (e.g. multilayered neural net or PCA-type dimensionality reduction) could be used for analysis. Nevertheless, we do not expect the neural mechanism to differ widely across apteronotid species so our analysis provides a meaningful comparison. The strength of our measure is that it is conservative: it gives a clear account of the information available in ELL pyramidal cells with few assumptions about what the downstream networks use for decoding.

\section{Trade-offs between specialization and generalization}

Classical neuroethology dictates that the mode of signal production and mode of signal reception must evolve in synchrony so that senders and receivers never lose the ability to exchange information (Bradbury and Vehrencamp, 2011). There are many examples of specialization of particular aspects of sensory systems to accomplish a highly specialized tasks (Endler, 1992). In the case of communication, sensory tuning for sender-receiver matching has been shown repeatedly. However, the converse may also be true. Over-specialization may come at the cost of reduced sensitivity to more general environmental signals. In such a case, it may be more beneficial to favor sensory generalization over specialization in animals that engage in social behaviors less often than their more gregarious relatives do. Indeed, there are several 
examples of peripheral sender-receiver mismatching that may be explained by gains in sensitivity to prey or predator signals to (Mason, 1991; Römer, 2016). While we see in $A$. leptorhynchus dedicated codes for communication signals with distinct meaning and in distinct contexts (Allen and Marsat, 2018; Marsat and Maler, 2010), maintaining that level of specificity for conspecific communication may be costly both metabolically (Niven and Laughlin, 2008) and in regards to detecting environmental stimuli apart from communication signals.

In $A$. albifrons we show a general match between signal characteristics, low frequency chirp envelopes, and CNS sensitivity to low frequency signals, but a lack of detailed coding that would allow for efficient discrimination of chirp identity in all contexts. Additional changes that would lead to a more accurate chirp coding could come at the expense of the ability to encode other stimuli. Therefore, for this species, limiting the resources dedicated to the coding of social signals in certain contexts (e.g. aggressive encounters with a fish with a high difference in EOD frequency) may allow the system to preserve or enhance sensitivity to other stimuli. 
Figures

A

$100 \mathrm{~ms}$

Chirp 1

Chirp 4

Chirp 7

Chirp 9
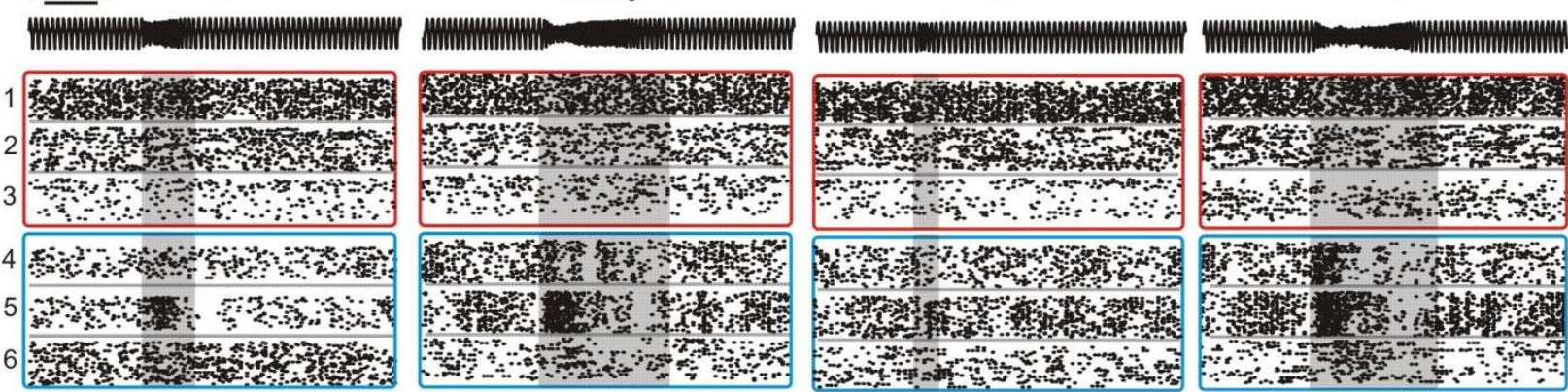

B
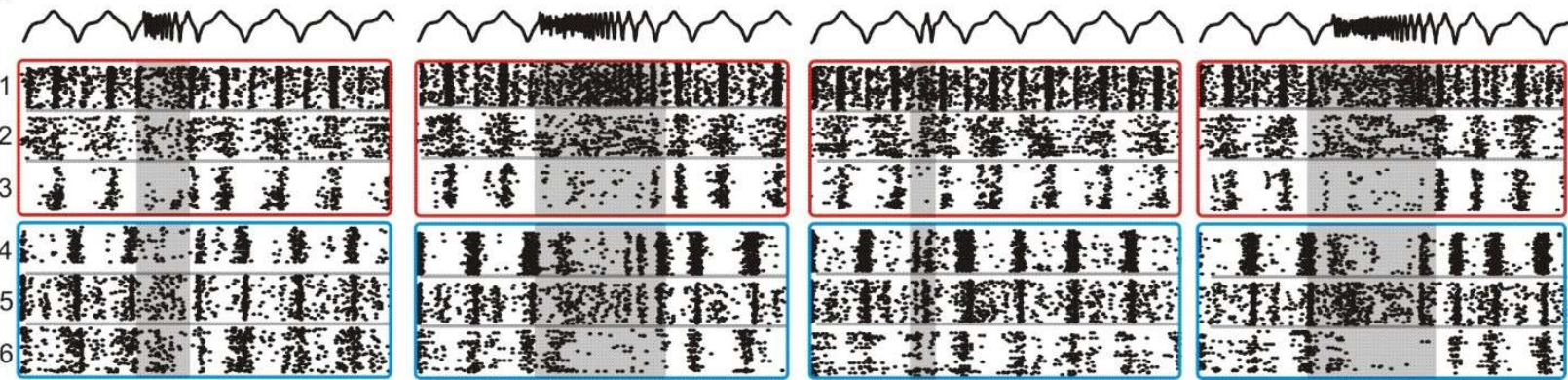

\section{Figure 1:}

ELL Pyramidal cell responses to conspecific signals. A. Raster plots of chirp responses on a high frequency $(100 \mathrm{~Hz})$ beat. AM of the EOD stimulus is shown in black; 3 representative OFFcells are shown in cyan boxes while 3 ON-cells responses are displayed in red boxes. The same 6 neurons are used for all panels. B. Responses to chirps on a low frequency $(10 \mathrm{~Hz})$ beat. For a detailed description of all chirps used, see Table 1 . The shaded area highlights the duration of the chirp. See Figure 1-1 and 1-2 for comments and analysis of specific response properties (adaptation time and biphasic responses to low frequencies). 
A

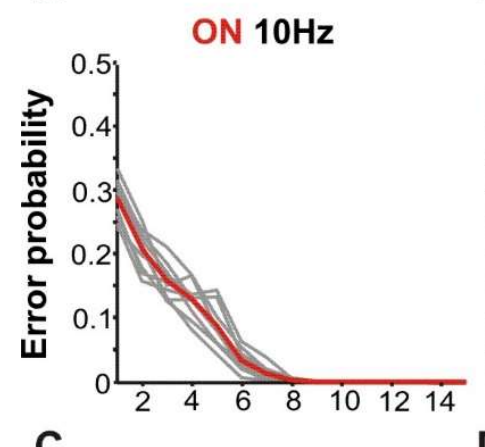

C OFF $10 \mathrm{~Hz}$

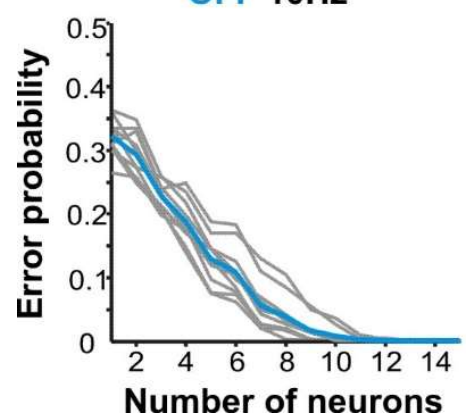

Number of neurons
B

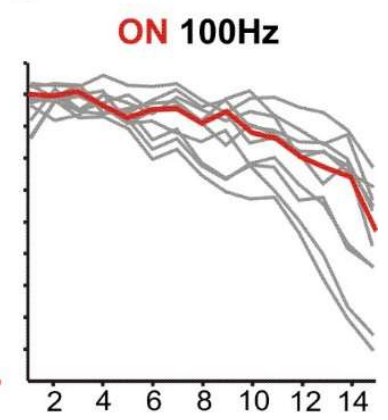

D OFF $100 \mathrm{~Hz}$

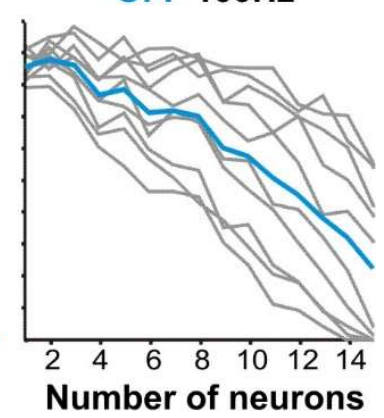

Figure 2:

Detection efficiency depends on beat frequency. A. Detection of chirps on $10 \mathrm{~Hz}$ beat by ONcells as a factor of neurons included in the analysis $(n=17)$. Error probability is the probability of an ideal observer to correctly assign a spike train as a chirp or beat response. Detection error levels for individual chirp identities are shown in gray. Red line indicates mean detection error for all chirps. ON-cells can reliably detect the occurrence of all chirps. B. ON-cell performance is worse on $100 \mathrm{~Hz}$ beats. C, D Mean OFF-cell performance (cyan, $n=16$ ) is also more efficient on a $10 \mathrm{~Hz}$ beat. 
A
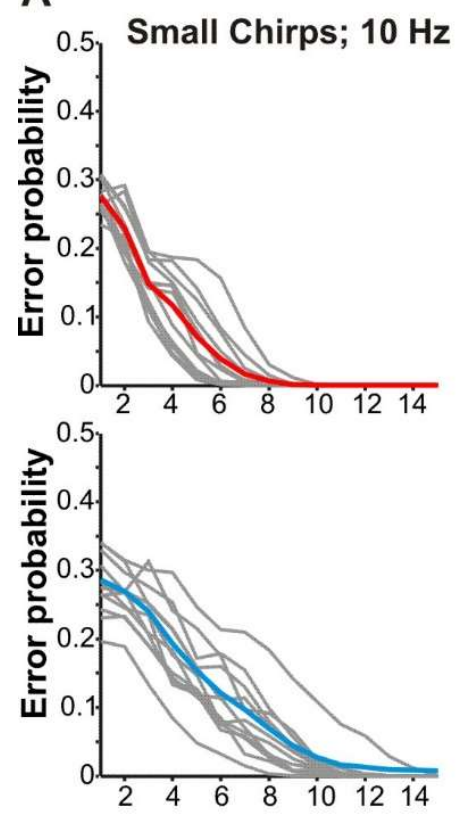

B Small Chirps; $100 \mathrm{~Hz}$
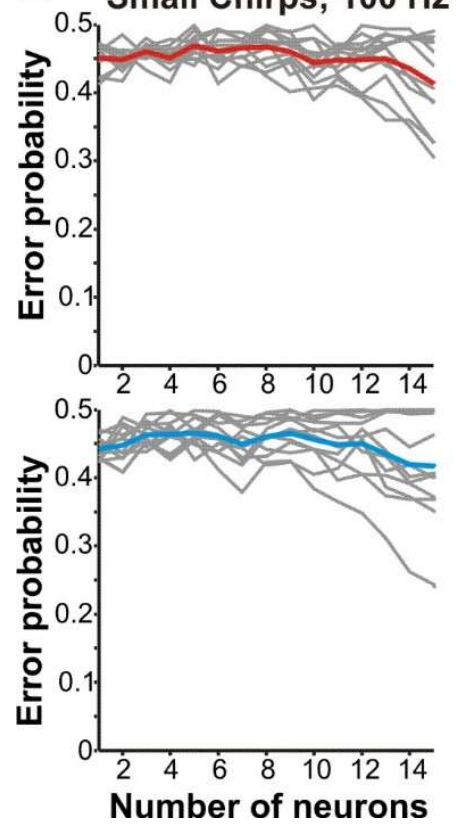

Big Chirps; $10 \mathrm{~Hz}$
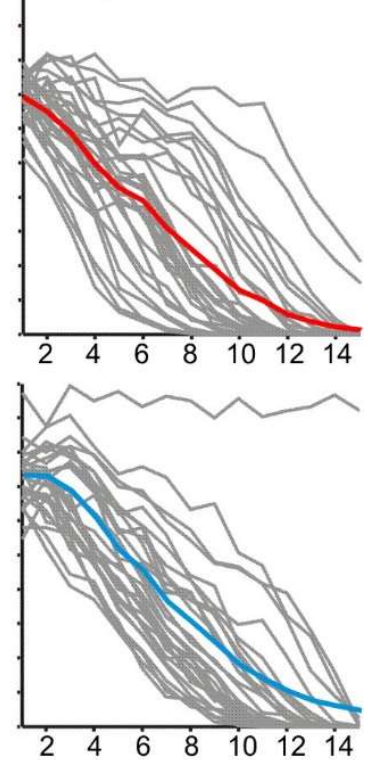

Big Chirps; $100 \mathrm{~Hz}$
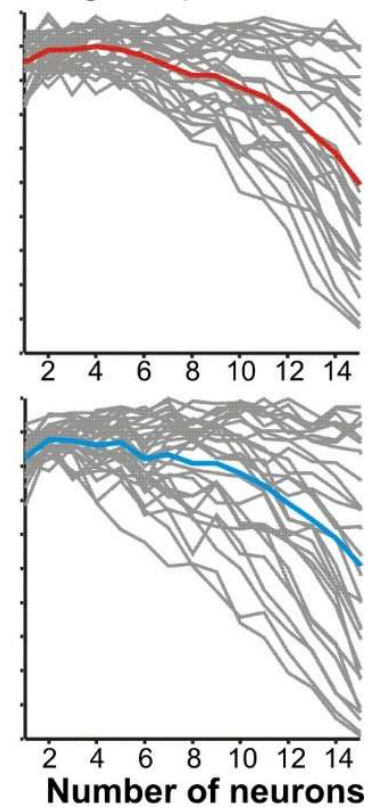

\section{Figure 3:}

Discrimination of chirps on high frequency beats is poor. A. Discrimination for small chirps (Chirps 5,6,7,8, right) and big chirps (Chirps 1,2,3,4,9,10, left) on $10 \mathrm{~Hz}$ beat. Mean ON-cell chirp discrimination shown in red, OFF-cells in cyan, and discrimination for individual chirp pairs shown in gray. $\mathbf{B}$. Discrimination of small chirps on a $100 \mathrm{~Hz}$ beat is relatively inefficient for both $\mathrm{ON}$ (red) and OFF-cells (cyan). Discrimination of big chirps varies with chirp identity, but is still poor. For chirp-by-chirp discrimination comparisons see Figure 3-1. 

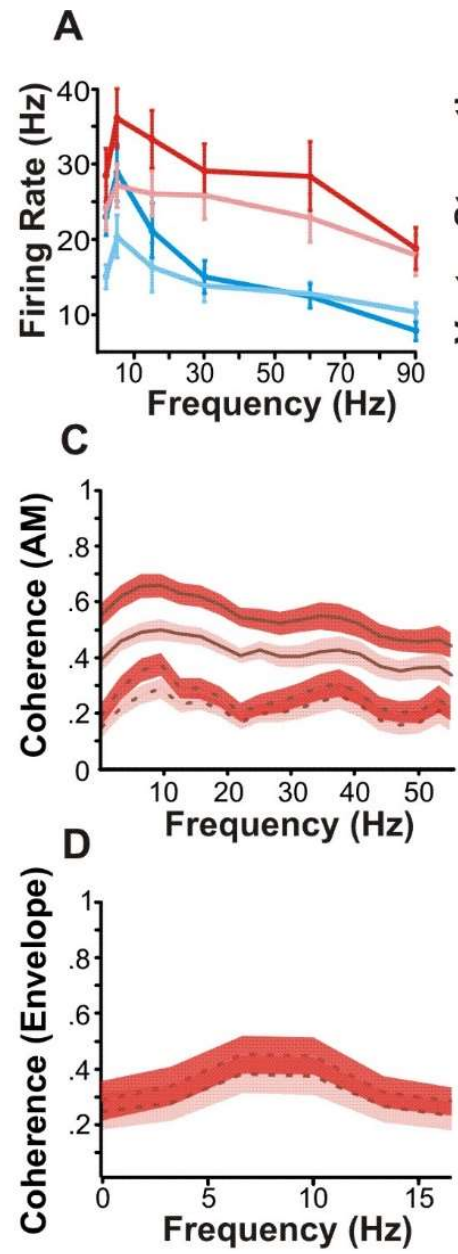
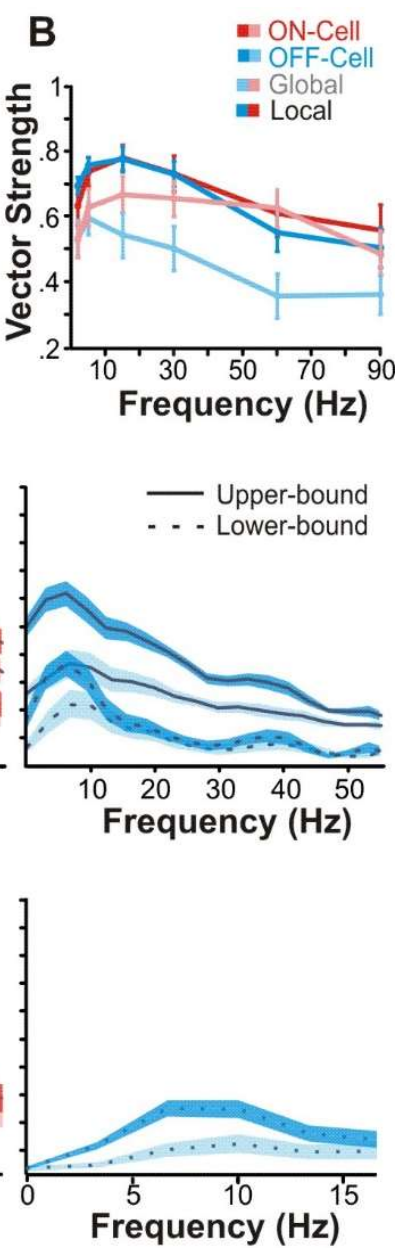

Figure 4:

Temporal coding properties of pyramidal cells A. Mean firing in response to SAM stimuli. Mean firing rate $( \pm$ s.e. $)$ for both $\mathrm{ON}$ (red, $n=19$ ) and OFF (cyan, $n=15$ ) cells in both stimulation configurations (global: pink, pale blue; local: red, cyan) peaks at $5 \mathrm{~Hz}$. B. Phase locking to AM sinusoids is also best at low frequencies. Maximum phase locking is seen at $15 \mathrm{~Hz}$, with the exception of locally stimulated OFF-cells, which peak at 5Hz. C. Mean coherence to noise stimulation is also low-pass. ON-cell coherence is shown in red, OFF-cell in cyan. The upper bound coherence measure (solid line) is based on the response-response correlations between multiple presentations of the stimulus, while the lower bound (dashed line) is based on stimulus-response correlations. Shaded areas indicate standard error; darker shading indicates local stimulation. Mean global lower bound maximums: ON-cells: $23.1 \mathrm{~Hz}$ ( \pm .86 s.e.); OFF-cells: $11.6 \mathrm{~Hz}( \pm .75$ s.e.), (Wilcoxon rank-sum test $\mathrm{p}=.04$ ). D. Coding of low frequency envelopes is poor. Mean $( \pm$ s.e.) lower bound coherence between

responses and the low-frequency $(0-20 \mathrm{~Hz})$ envelope of a bandpass RAM stimulus $(40-60 \mathrm{~Hz})$ are displayed for both local and global stimulus configurations. Both ON and OFF cells exhibit peak envelope tuning at $10.10 \mathrm{~Hz}( \pm 1.52$ s.e.) (Wilcoxon rank-sum test, $p=.21)$. OFF-cells have noticeably lower coherence to low envelopes than ON cells. 
A

A. leptorhynchus Chirp

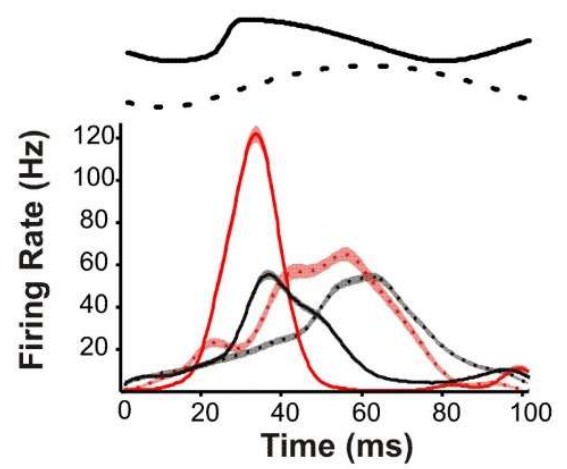

D

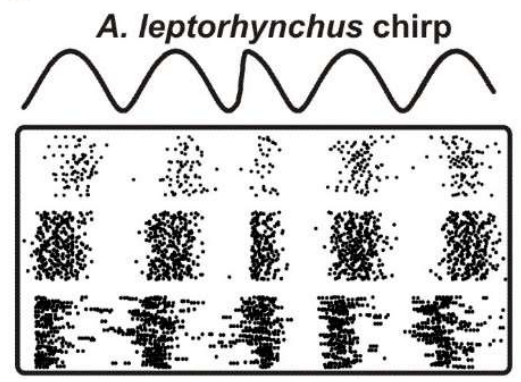

B

\section{A. albifrons Chirp}

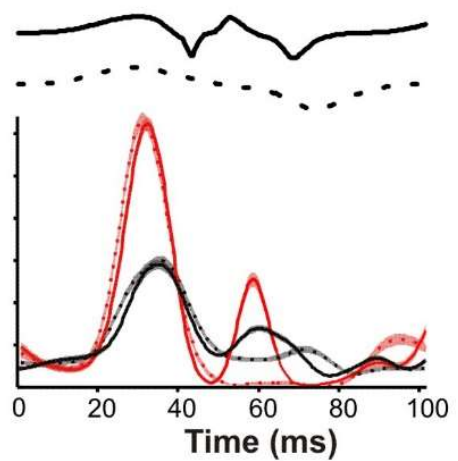

A. leptorhynchus chirp

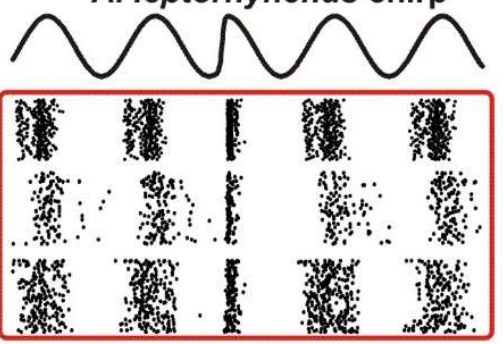

C
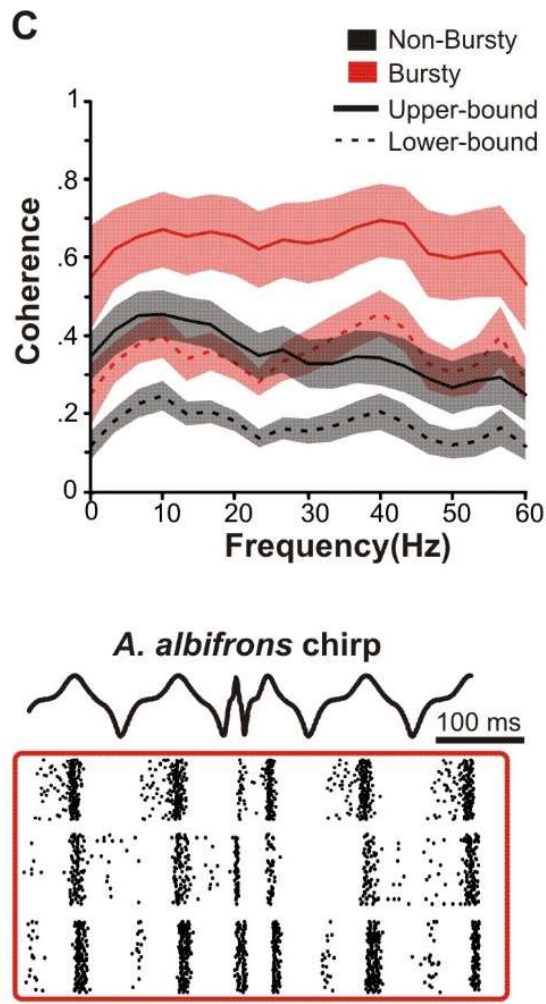

Figure 5:

Coding of chirps by a small population of higher-pass neurons. A. The mean instantaneous firing rates of ON-cells over the time course of chirp and beat stimuli. The difference in peak firing rate characterize a small population $(\mathrm{n}=5$, red) that bursts in response to A. leptorhynchus small chirps (Beat responses, dashed lines: Peak FR 77.67 HZ \pm 7.11 ; Chirp responses, solid lines: Peak FR 159.57 Hz \pm 8.68 ; Wilcoxon ranked sum test $(\mathrm{p}<0.001)$. The majority of the ONcell population ( $\mathrm{n}=16$, black) only showed a modest increase in firing (Beat Response: peak FR $62.68 \mathrm{~Hz} \pm 1.27$; chirp response: peak FR $66.56 \mathrm{~Hz} \pm 4.21$, Wilcoxon ranked sum test $\mathrm{p}=.002$ ). Shaded area represents standard error. B. Even the bursty population fires less in response to $A$. albifrons smallest chirps compared to the beat (beat response: peak FR $155.45 \mathrm{~Hz} \pm 8.82$; chirp response: peak FR $117.75 \mathrm{~Hz} \pm 9.40$, Wilcoxon ranked sum test $(\mathrm{p}=.04))$. C. Mean $( \pm$ s.e. $)$ upperbound and lower-bound coherences for the bursting (red) and non-bursting (black) populations. . D. Example raster plots of chirp responses used for A and B. The non-bursty population (black box) responds similarly to both A. leptorhynchus chirp and beat. The bursty population (red box) bursts to $A$. leptorhynchus chirps, but not $A$. albifrons chirps.). For comparable data obtained from A. leptorhynchus see Figure 5-1. 

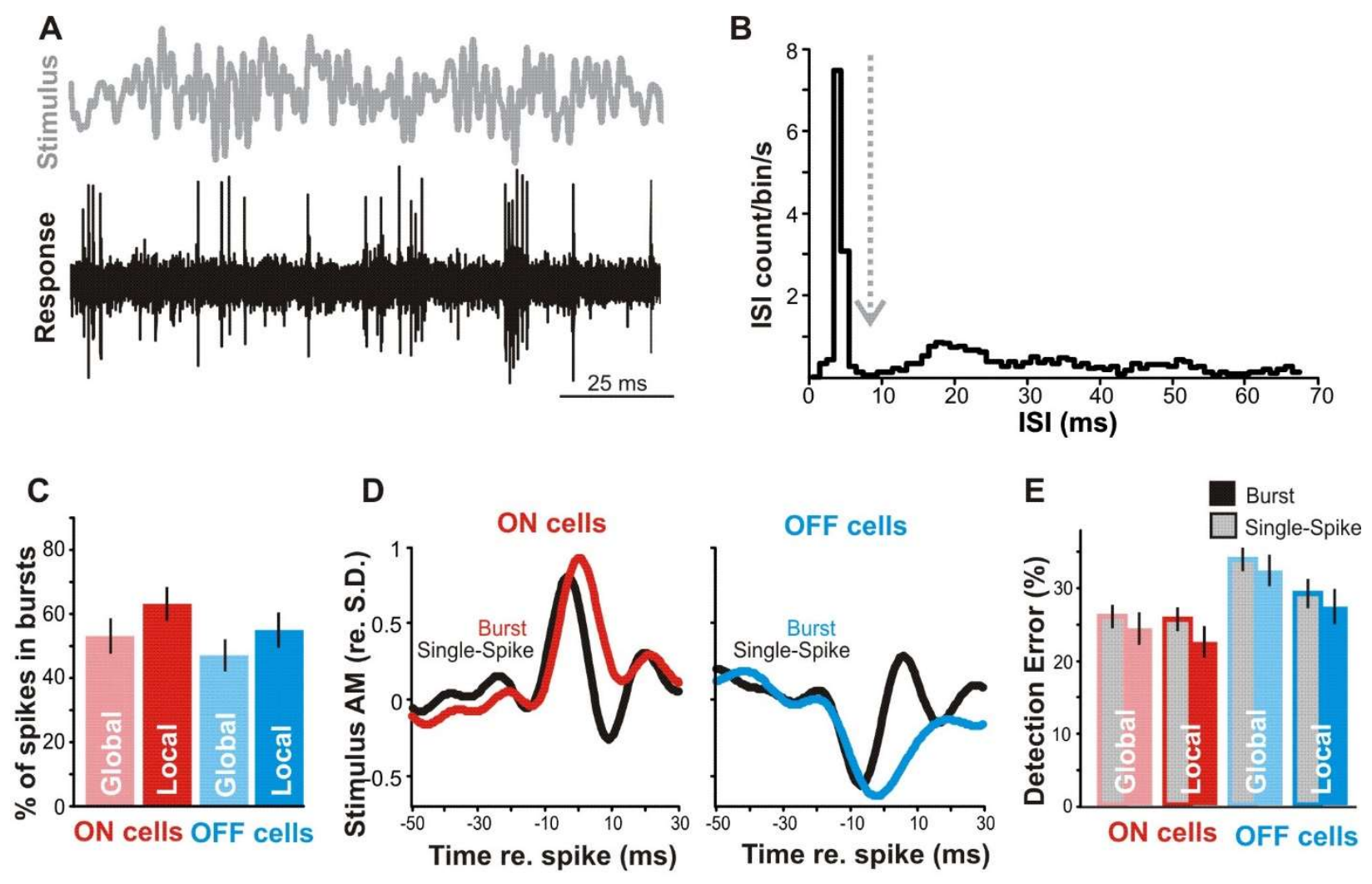

Figure 6:

Coding of AM by bursts. A. Sample of noise stimulus (0-60Hz, gray) and representative spike train (black) from an OFF-cell during local stimulation. B. Example of ISI distribution used to determine burst threshold (dashed line). C. ON-cells (red) burst more than OFF-cells (blue) (ANOVA, $\mathrm{p}=.02$ ), and local stimulation produced more bursting than global (ANOVA, $\mathrm{p}=.01$ ). Error bars show standard error D. Mean burst triggered averages (red/blue) and single spike triggered averages (black) from ON and OFF-cells show that bursts are triggered by wider (lower-frequency) stimulus features than single spikes. E. Feature detection performance for burst and isolated spikes. In both ON and OFF cells bursts (blue/red -filled bars) tend to have lower error rates (percentage of events signaling false positives or false negatives) in detecting optimal stimulus features than single spikes (gray-filled bars) but this trend is not significant (ANOVA, $p=.10)$. 

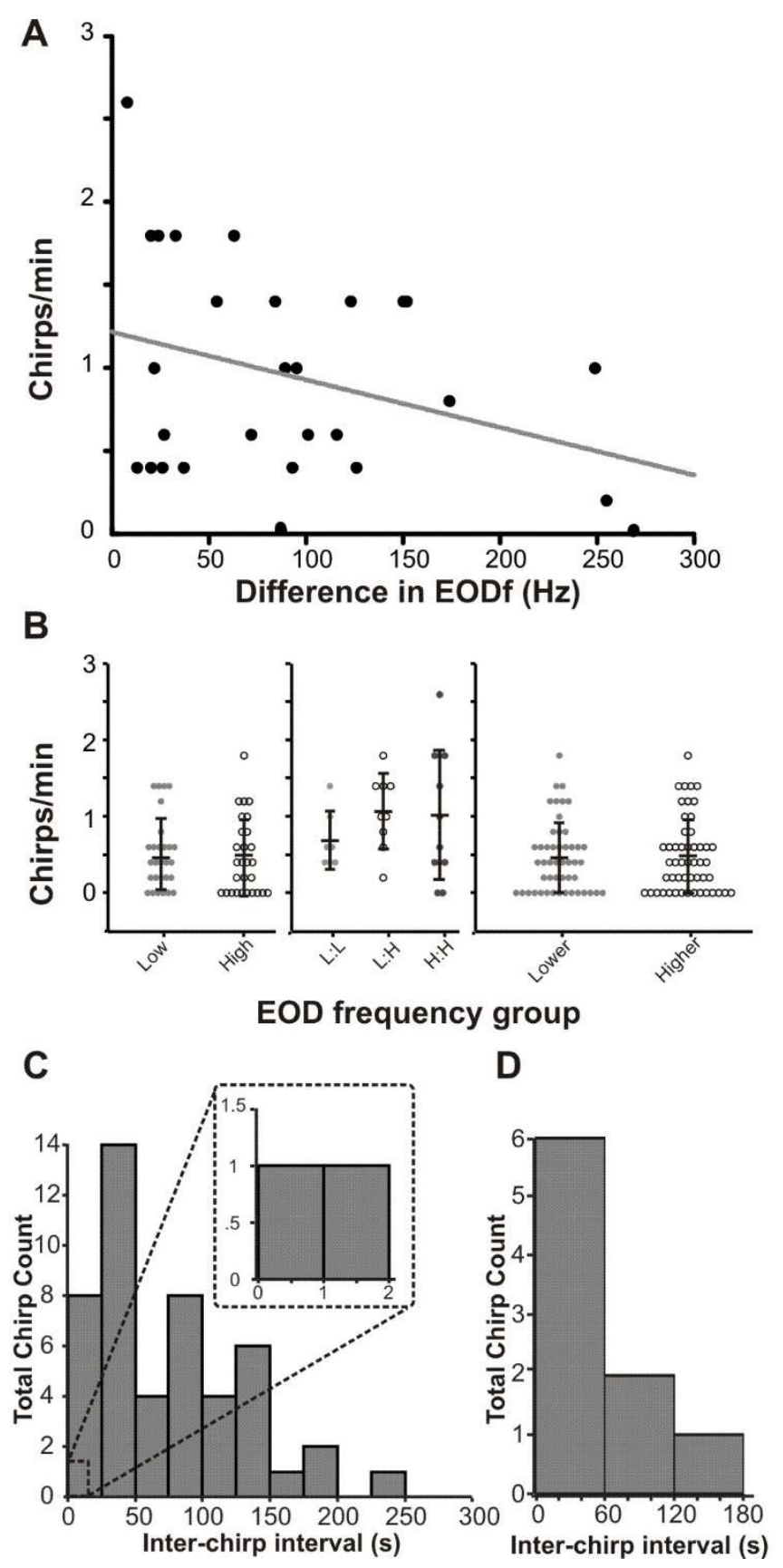

Figure 7:

Chirping behavior in freely swimming pairs $(\mathrm{n}=28)$. A. The number of chirps produced during interaction is correlated with difference in EODf $\left(\mathrm{r}^{2}=.1093\right)$ B. Chirping does not differ by absolute EODf, EODf pair type or relative EODf. Mean chirp rate for low frequency $(<1100 \mathrm{~Hz})$ and high frequency fish $(>1100)$ was similar (Student's t-test, $\mathrm{p}=.86$ ). Mean chirps per trial based on EODf of pairing (Low: Low, Low: High, High: High) (ANOVA, p=.47), and by relative frequency of individuals within the pairing (Student's $t$ test, $\mathrm{p}=.55$ ) were all extremely low and similar in all groupings. Error bars indicate standard deviation. C. Inter-chirp intervals between pairs binned by time fall show no echoing chirp exchanges. Inset: Enlarged section show that very few chirps occur within $2 \mathrm{~s}$ of each other. D. Inter-chirp intervals distribution for individual fish follow a Poisson distribution $\left(\mathrm{R}^{2}=0.9\right)$. 
Table 1

Table 1: Frequency and duration properties for all chirps used. "Other" indicates differences in chirp shape not due to peak frequency or duration, such as shape of frequency rise and fall, and phase of the beat on which the chirp occurred.

\begin{tabular}{|c|c|c|c|}
\hline Chirp ID & Frequency Rise $(\mathrm{Hz})$ & Duration (ms) & Other \\
\hline 1 & 200 & 100 & $\alpha$ shape \\
\hline 2 & 200 & 200 & $\alpha$ shape \\
\hline 3 & 350 & 100 & $\alpha$ shape \\
\hline 4 & 350 & 200 & $\alpha$ shape \\
\hline 5 & 50 & 50 & $\alpha$ shape \\
\hline 6 & 100 & 50 & $\alpha$ shape \\
\hline 7 & 50 & 50 & Antiphase to 5 \\
\hline 8 & 100 & 50 & Antiphase to 6 \\
\hline 9 & 350 & 200 & Two frequency peaks \\
\hline 10 & 350 & 200 & Ramp Shaped \\
\hline 11 & 60 & 10 & A. lept. small chirp \\
\hline 12 & 122 & 15 & A. lept. small chirp \\
\hline
\end{tabular}

Extended Data 

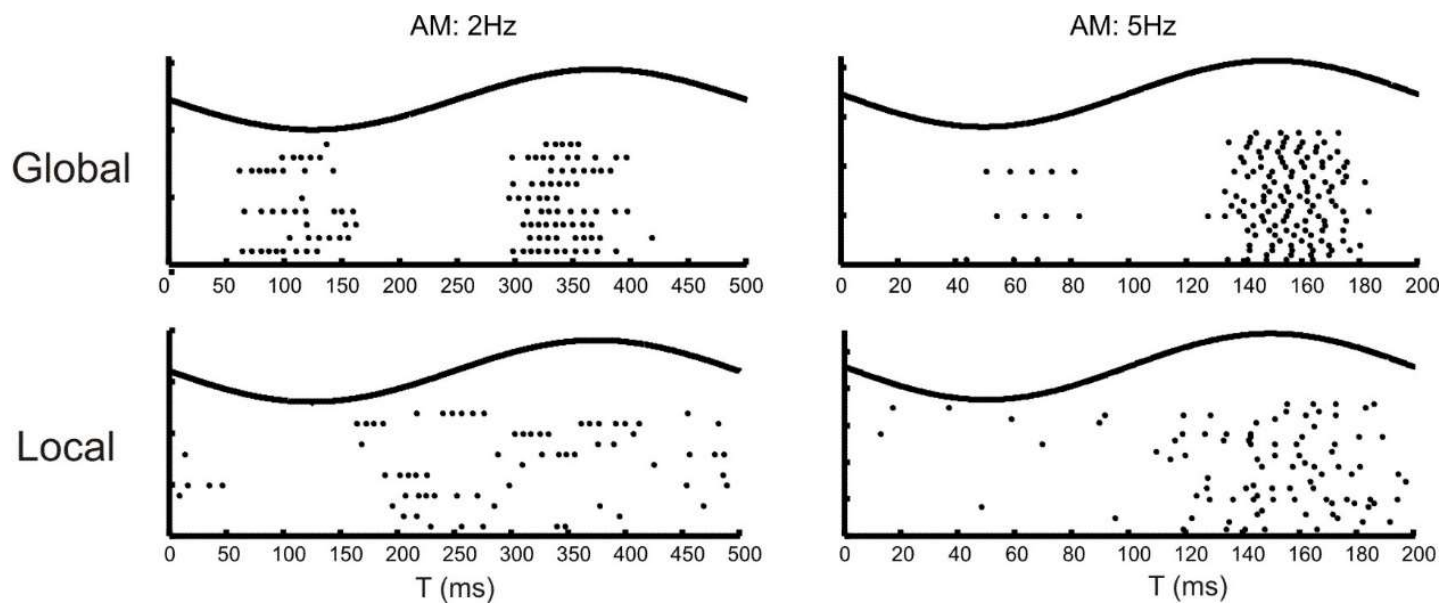

\section{Figure 1-1:}

Cancellation observed in an LS ON cell. In some recordings a biphasic response to stimuli is observed. While this could indicate an overly strong stimulus, it could also indicate cancellation via feedback from the midbrain (Bastian et al., 2004). Cancellation is less prominent in the LS of A. leptorhynchus, but still occurs. The biphasic responses to global SAM stimuli but not to local stimulation indicate that our observed responses likely represent an increased cancellation response in the LS as a result of low frequency tuning. 
A

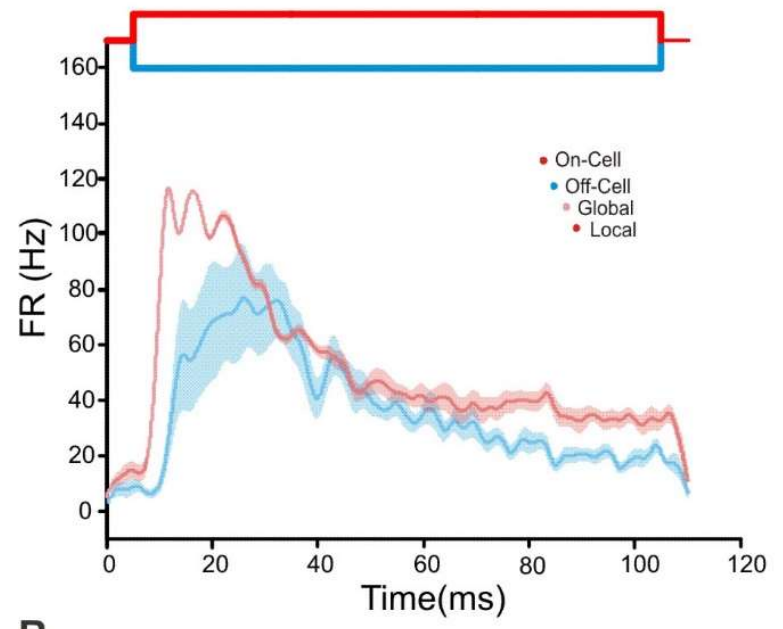

B

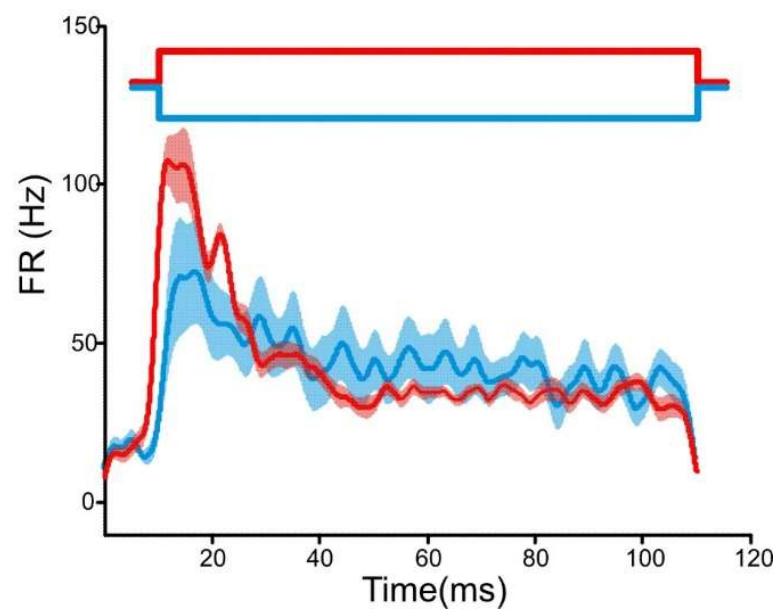

Figure 1-2:

A. albifrons adaptation to step stimulation. A. Both ON and OFF cells' responses decreased throughout the $100 \mathrm{~ms}$ stimulus without plateauing in sharp contrast with B. A. leptorhynchus responses to global stimuli (S2, Krahe et al., 2008 see their figure 8C\&F) that adapt within 40$50 \mathrm{~ms}$ and then plateau. 
A
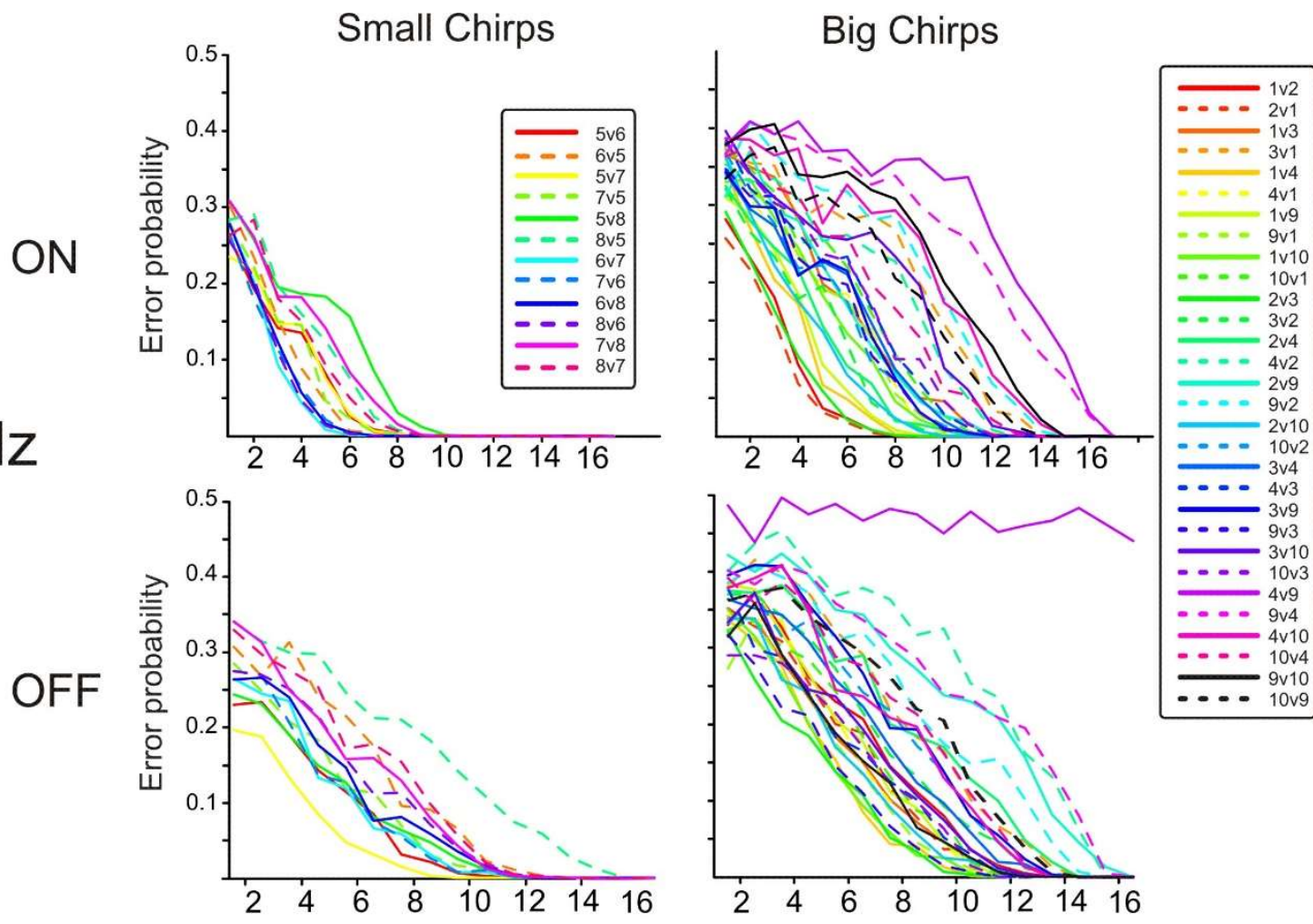

B

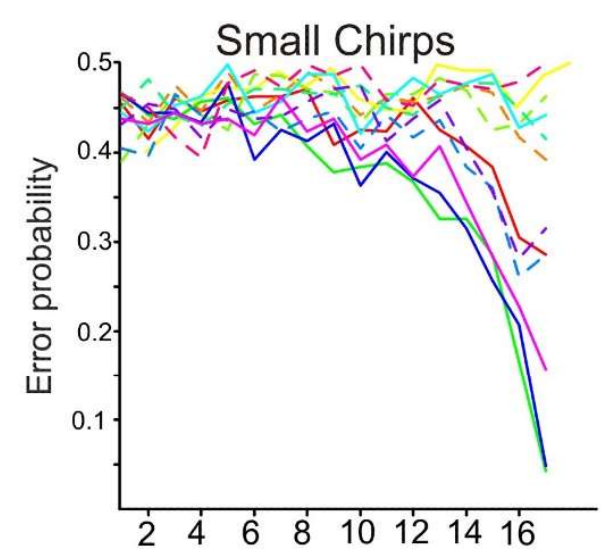

$100 \mathrm{~Hz}$
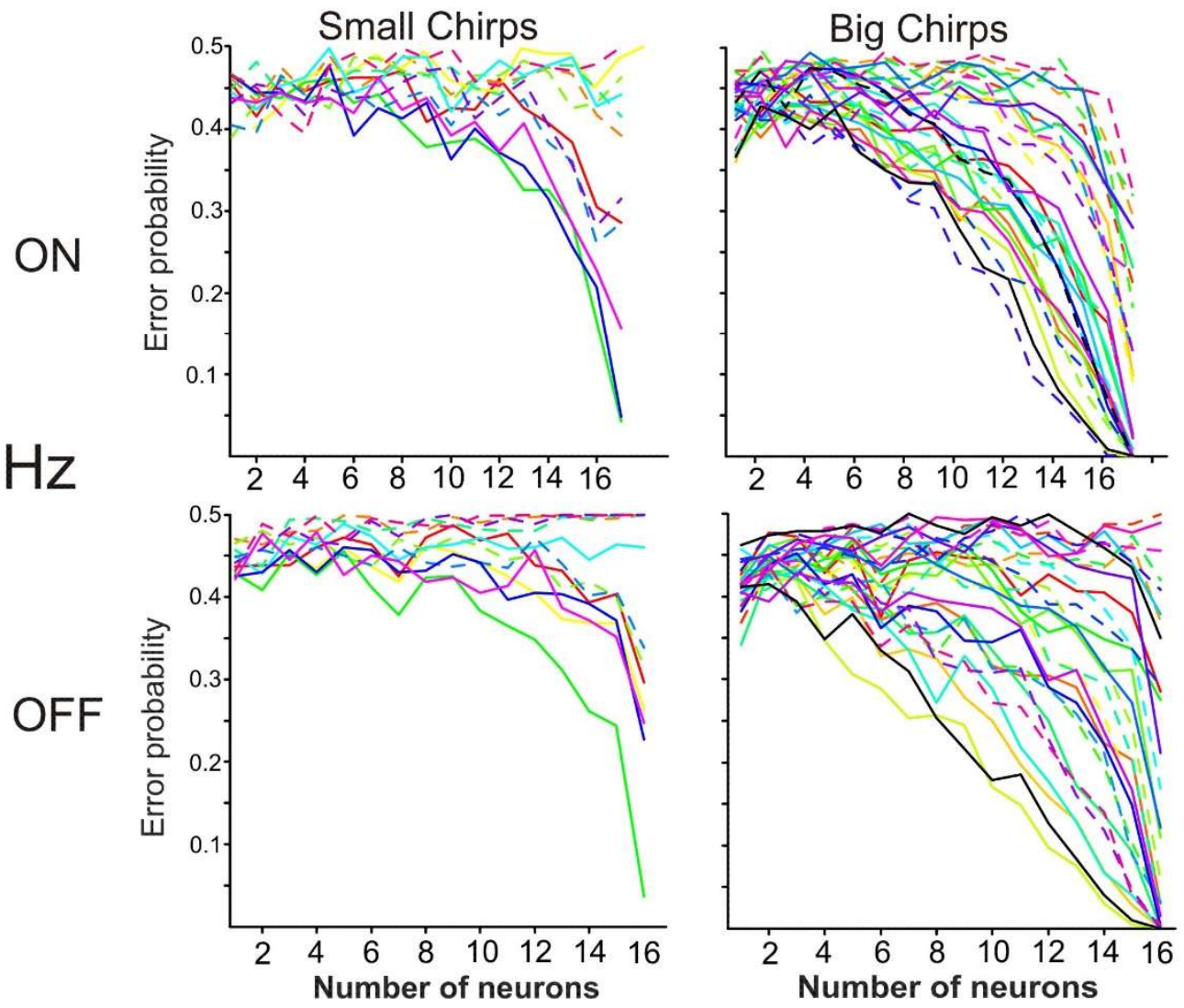
Figure 3-1:

Detailed discrimination traces indicating results of testing specific chirps against each other. For ease of analysis, chirps were grouped by duration frequency and duration as either big or small. Duration appears to be the feature that is most discriminable, so big and small chirps were not directly compared. Descriptions of chirp properties are located in Table 1. A. Chirp discrimination on a $10 \mathrm{~Hz}$ beat. B) Chirp discrimination on a $100 \mathrm{~Hz}$ beat. 

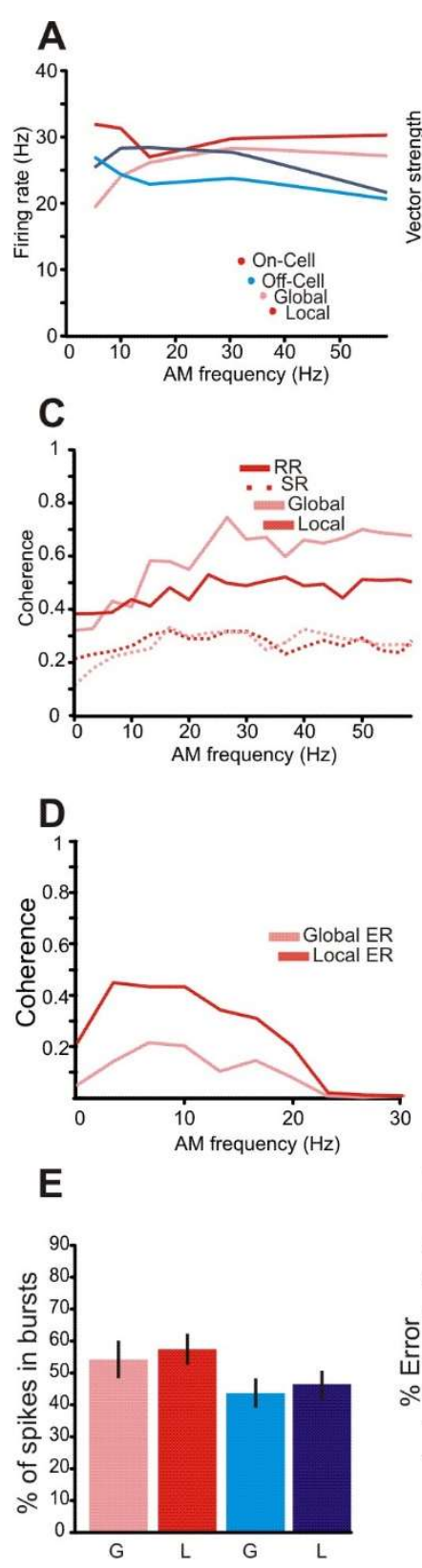

to sinusoids across $0-60 \mathrm{~Hz}$ is best at $20-30$
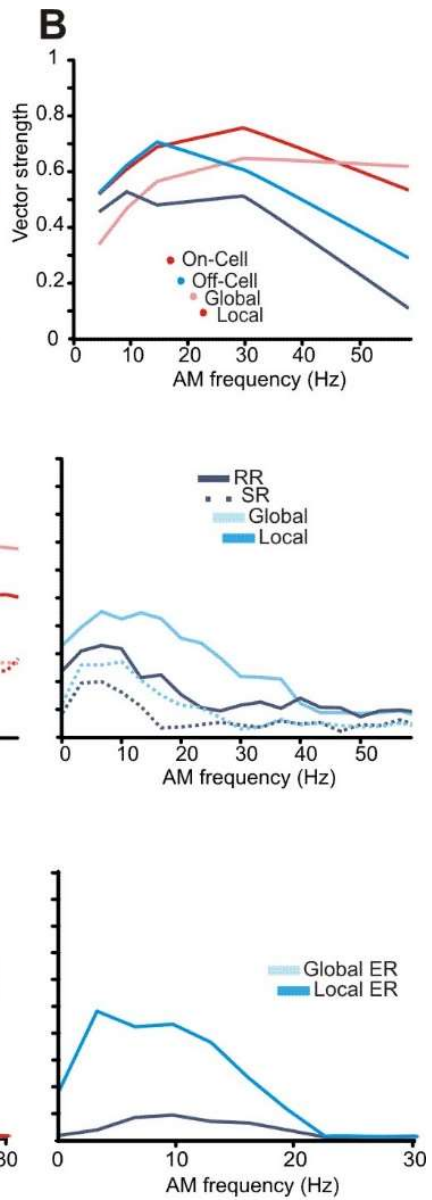

$\mathbf{F}$

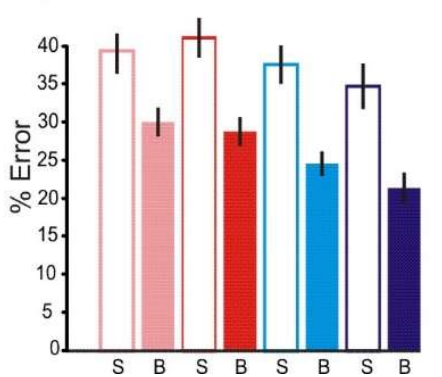

Figure 5-1:

A. leptorhynchus data for stimulation protocols matched to those used in the main text. This data is comparable to what has already be extensively published in Krahe et al. (Krahe et al., 2008), and thus not highlighted in the main text, but indicate that our methods reproduce previously published results and indicate differences between $A$.

albifrons and A. leptorhynchus. A. Coherence to $0-60 \mathrm{~Hz}$ RAM stimulation. Mean

coherence to noise stimulation by neuron type. ON-cell coherence is shown in red, OFF-cell in cyan. The upper bound coherence measure is shown with solid lines, lower bound in dotted lines B. Phase locking orz, while firing rate is relatively constant across frequencies C. Coherence to low frequency envelopes. Both ON and OFF cells perform well at coding low frequency stimulus components. 


\section{Works Cited}

Allen, K. M. and Marsat, G. (2018). Task-specific sensory coding strategies matched to detection and discrimination behaviors in Apteronotus leptorhynchus. J. Exp. Biol. 221,.

Aumentado-Armstrong, T., Metzen, M. G., Sproule, M. K. J. and Chacron, M. J. (2015). Electrosensory Midbrain Neurons Display Feature Invariant Responses to Natural Communication Stimuli. PLoS Comput. Biol. 11, 1-28.

Ávila-Åkerberg, O., Krahe, R. and Chacron, M. J. (2010). Neural heterogeneities and stimulus properties affect burst coding in vivo. Neuroscience 168, 300-313.

Bastian, J. (1981). II. The effects of moving objects and other electrical stimuli on the activities of two categories of posterior lateral line lobe cells in Apteronotus albifrons. J. Comput. Physiol. 144, 481-494.

Bastian, J. (1986). Gain control in the electrosensory system: a role for the descending projections to the electrosensory lateral line lobe. J. Comp. Physiol. A 158, 505-515.

Bastian, J., Chacron, M. J. and Maler, L. (2004). Plastic and nonplastic pyramidal cells perform unique roles in a network capable of adaptive redundancy reduction. Neuron 41, 767-779.

Benda, J., Longtin, A. and Maler, L. (2005). Spike-frequency adaptation separates transient communication signals from background oscillations. J. Neurosci. 25, 2312-21.

Benda, J., Longtin, A. and Maler, L. (2006). A synchronization-desynchronization code for natural communication signals. Neuron 52, 347-358.

Borst, A. and Theunissen, F. E. (1999). Information theory and neural coding. Nat. Neurosci. 2, 947-957.

Bradbury, J. W. and Vehrencamp, S. L. (2011). Principles of animal communication. 2nd ed. Sunderland, Mass., Mass.: Sinauer Associates.

Brumm, H. and Slabbekoorn, H. (2005). Acoustic Communication in Noise. Adv. Study Behav.

Chacron, M. J. (2006). Nonlinear Information Processing in a Model Sensory System. J. Neurophysiol. 95, 2933-2946.

Chacron, M. J. and Bastian, J. (2008). Population coding by electrosensory neurons. $J$. Neurophysiol. 99, 1825-1835.

Chacron, M. J., Maler, L. and Bastian, J. (2005). Feedback and feedforward control of frequency tuning to naturalistic stimuli. J. Neurosci. 25, 5521-5532.

Creutzig, F., Wohlgemuth, S., Stumpner, A., Benda, J., Ronacher, B. and Herz, A. V. M. (2009). Timescale-Invariant Representation of Acoustic Communication Signals by a Bursting Neuron. J. Neurosci. 29, 2575.

Deemyad, T., Metzen, M. G., Pan, Y. and Chacron, M. J. (2013). Serotonin selectively enhances perception and sensory neural responses to stimuli generated by same-sex conspecifics. Proc. Natl. Acad. Sci. U. S. A. 110, 19609-14. 
Dunlap, K. D. and Larkins-Ford, J. (2003). Diversity in the structure of electrocommunication signals within a genus of electric fish, Apteronotus. J. Comp. Physiol. A 189, 153-161.

Dunlap, K. D., Thomas, P. and Zakon, H. H. (1998). Diversity of sexual dimorphism in electrocommunication signals and its androgen regulation in a genus of electric fish, Apteronotus. J. Comp. Physiol. A Sensory, Neural, Behav. Physiol. 183, 77-86.

Endler, J. A. (1992). Signals, Signal Conditions, and the Direction of Evolution. Am. Nat. 139, S125-S153.

Frank, K. and Becker, M. C. (1964). Microelectrodes for recording and stimulation. In Physical techniques in biological research (ed. Nastuk, W. L.), pp. 23-84. New York: Academic Press.

Fujimoto, H., Hasegawa, T. and Watanabe, D. (2011). Neural Coding of Syntactic Structure in Learned Vocalizations in the Songbird. J. Neurosci. 31, 10023.

Gabbiani, F., Metzner, W., Wessel, R. and Koch, C. (1996). From stimulus encoding to feature extraction in weakly electric fish. Nature 384, 564-567.

Gerhardt, H. C. and Schwartz, J. J. (2001). Auditory tuning and frequency preferences. Anuran Commun. Smithson. Inst. Press. Washingt.

Goldberg, J. M. and Brown, P. B. (1969). Response of binaural neurons of dog superior olivary complex to dichotic tonal stimuli: some physiological mechanisms of sound localization. $J$. Neurophysiol. 32, 613-636.

Hagedorn, M. and Heiligenberg, W. (1985). Court and spark: electric signals in the courtship and mating of gymnotoid fish. Anim. Behav. 33, 254-265.

Harris-Warrick, R. M. and Marder, E. (1991). Modulation of neural networks for behavior. Annu. Rev. Neurosci. 14, 39-57.

Henninger, J., Krahe, R., Kirschbaum, F., Grewe, J. and Benda, J. (2018). Statistics of natural communication signals observed in the wild identify important yet neglected stimulus regimes in weakly electric fish. J. Neurosci. 0350-18.

Hofmann, V. and Chacron, M. J. (2017). Differential receptive field organizations give rise to nearly identical neural correlations across three parallel sensory maps in weakly electric fish. PLoS Comput. Biol. 13, e1005716.

Houghton, C. and Sen, K. (2008). A New Multineuron Spike Train Metric. Neural Comput. 20, 1495-1511.

Hupé, G. J. (2012). Electrocommunication in a Species of Weakly Electric Fish Apteronotus Leptorhynchus: Signal Patterning and Behaviour.

Hupé, G. J. and Lewis, J. E. (2008). Electrocommunication signals in free swimming brown ghost knifefish, Apteronotus leptorhynchus. J. Exp. Biol. 211, 1657-1667.

Itatani, N. and Klump, G. M. (2014). Neural correlates of auditory streaming in an objective behavioral task. Proc. Natl. Acad. Sci. 111, 10738 LP-10743. 
Kolodziejski, J., Sanford, S. E. and Smith, G. T. (2007). Stimulus frequency differentially affects chirping in two species of weakly electric fish: implications for the evolution of signal structure and function. J. Exp. Biol. 210, 2501-2509.

Krahe, R., Bastian, J. and Chacron, M. J. (2008). Temporal processing across multiple topographic maps in the electrosensory system. J. Neurophysiol. 100, 852-67.

Larson, E., Billimoria, C. P. and Sen, K. (2009). A biologically plausible computational model for auditory object recognition. $J$. Neurophysiol. 101, 323-331.

Larson, E., Perrone, B. P., Sen, K. and Billimoria, C. P. (2010). A Robust and Biologically Plausible Spike Pattern Recognition Network. J. Neurosci. 30, 15566 LP-15572.

Machens, C. K., Schütze, H., Franz, A., Kolesnikova, O., Stemmler, M. B., Ronacher, B., Herz, A. V. M., Schutze, H., Franz, A., Kolesnikova, O., et al. (2003). Single auditory neurons rapidly discriminate conspecific communication signals. Nat. Neurosci. 6, 341342.

Maler, L., Sas, E., Johnston, S. and Ellis, W. (1991). An atlas of the brain of the electric fish Apteronotus leptorhynchus. J. Chem. Neuroanat. 4, 1-38.

Marsat, G. and Maler, L. (2010). Neural heterogeneity and efficient population codes for communication signals. J. Neurophysiol. 104, 2543-55.

Marsat, G. and Pollack, G. S. (2004). Differential temporal coding of rhythmically diverse acoustic signals by a single interneuron. J. Neurophysiol. 92, 939-48.

Marsat, G., Proville, R. D. and Maler, L. (2009). Transient signals trigger synchronous bursts in an identified population of neurons. J. Neurophysiol. 102, 714-723.

Martinez-Conde, S., Macknik, S. L. and Hubel, D. H. (2002). The function of bursts of spikes during visual fixation in the awake primate lateral geniculate nucleus and primary visual cortex. Proc. Natl. Acad. Sci. U. S. A. 99, 13920-13925.

Martinez, D., Metzen, M. G. and Chacron, M. J. (2016). Electrosensory processing in Apteronotus albifrons: implications for general and specific neural coding strategies across wave-type weakly electric fish species. J. Neurophysiol. 116, 2909-2921.

Mason, A. C. (1991). Hearing in a primitive ensiferan: the auditory system of Cyphoderris monstrosa (Orthoptera: Haglidae). J. Comp. Physiol. A 168, 351-363.

Metzen, M. G., Huang, C. G. and Chacron, M. J. (2018). Descending pathways generate perception of and neural responses to weak sensory input. PLOS Biol. 16, e2005239.

Metzner, W. (1999). Neural circuitry for communication and jamming avoidance in gymnotiform electric fish. J. Exp. Biol. 202, 1365-1375.

Metzner, W. and Heiligenberg, W. (1991). The coding of signals in the electric communication of the gymnotiform fish Eigenmannia: From electroreceptors to neurons in the torus semicircularis of the midbrain. J. Comp. Physiol. A 169, 135-150.

Metzner, W. and Juranek, J. (1997). A sensory brain map for each behavior? Proc. Natl. Acad. Sci. 94,. 
Metzner, W. and Viete, S. (1996). The Neuronal Basis of Communication and Orientation in the Weakly Electric Fish, EigenmanniaI. Communication Behavior or: Seeking a Conspecific?s Response. Naturwissenschaften 83, 6-14.

Middleton, J. W., Longtin, A., Benda, J. and Maler, L. (2006). The cellular basis for parallel neural transmission of a high-frequency stimulus and its low-frequency envelope. Proc. Natl. Acad. Sci. U. S. A. 103, 14596-601.

Mouterde, S. C., Elie, J. E., Mathevon, N. and Theunissen, F. E. (2017). Single Neurons in the Avian Auditory Cortex Encode Individual Identity and Propagation Distance in Naturally Degraded Communication Calls. J. Neurosci. 37, 3491 LP-3510.

Neuhofer, D. and Ronacher, B. (2012). Influence of Different Envelope Maskers on Signal Recognition and Neuronal Representation in the Auditory System of a Grasshopper. PLoS One 7, e34384.

Neuhofer, D., Wohlgemuth, S., Stumpner, A. and Ronacher, B. (2008). Evolutionarily conserved coding properties of auditory neurons across grasshopper species. Proc. R. Soc. B Biol. Sci. 275, 1965-1974.

Niven, J. E. and Laughlin, S. B. (2008). Energy limitation as a selective pressure on the evolution of sensory systems. J. Exp. Biol. 211, 1792 LP-1804.

Oswald, A.-M. M., Chacron, M. J., Doiron, B., Bastian, J. and Maler, L. (2004). Parallel processing of sensory input by bursts and isolated spikes. J. Neurosci. 24, 4351-4362.

Petzold, J. M., Marsat, G. and Smith, G. T. (2016). Co-adaptation of electric organ discharges and chirps in South American ghost knifefishes (Apteronotidae). J. Physiol.

Petzold, J. M., Alves-Gomes, J. A. and Smith, G. T. (2018). Chirping and asymmetric jamming avoidance responses in the electric fish \&lt;em\&gt;Distocyclus conirostris\&lt;/em\&gt; J. Exp. Biol. 221, jeb178913.

Römer, H. (2016). Matched Filters in Insect Audition: Tuning Curves and Beyond BT - The Ecology of Animal Senses: Matched Filters for Economical Sensing. In (ed. von der Emde, G.) and Warrant, E.), pp. 83-109. Cham: Springer International Publishing.

Saunders, J. and Bastian, J. (1984). The physiology and morphology of two types of electrosensory neurons in the weakly electric fish Apteronotus leptorhynchus. J. Comp. Physiol. A 154, 199-209.

Savard, M., Krahe, R. and Chacron, M. J. (2011). Neural heterogeneities influence envelope and temporal coding at the sensory periphery. Neuroscience 172, 270-284.

Schul, J. and Bush, S. L. (2002). Non-parallel coevolution of sender and receiver in the acoustic communication system of treefrogs. Proc. R. Soc. London. Ser. B Biol. Sci. 269, 1847 LP1852.

Smith, G. T. (2013). Evolution and hormonal regulation of sex differences in the electrocommunication behavior of ghost knifefishes (Apteronotidae). J. Exp. Biol. 216, 2421 LP-2433. 
Smith, A. R., Proffitt, M. R., Ho, W. W., Mullaney, C. B., Maldonado-Ocampo, J. A., Lovejoy, N. R., Alves-Gomes, J. A. and Smith, G. T. (2016). Evolution of electric communication signals in the South American ghost knifefishes (Gymnotiformes: Apteronotidae): A phylogenetic comparative study using a sequence-based phylogeny. $J$. Physiol. 110, 302-313.

Stamper, S. A., Carrera-G, E., Tan, E. W., Fugère, V., Krahe, R. and Fortune, E. S. (2010). Species differences in group size and electrosensory interference in weakly electric fishes: Implications for electrosensory processing. Behav. Brain Res. 207, 368-376.

Stamper, S. A., Fortune, E. S. and Chacron, M. J. (2013). Perception and coding of envelopes in weakly electric fishes. J. Exp. Biol. 216, 2393 LP-2402.

Stöckl, A., Sinz, F., Benda, J. and Grewe, J. (2014). Encoding of social signals in all three electrosensory pathways of Eigenmannia virescens. J. Neurophysiol. 112, 2076-2091.

Tootoonian, S., Coen, P., Kawai, R. and Murthy, M. (2012). Neural Representations of Courtship Song in the Drosophila Brain. J. Neurosci. 32, 787 LP-798.

Turner, C. R., Derylo, M., de Santana, C. D., Alves-Gomes, J. a and Smith, G. T. (2007). Phylogenetic comparative analysis of electric communication signals in ghost knifefishes (Gymnotiformes: Apteronotidae). J. Exp. bBology 210, 4104-4122.

van Rossum, M. C. (2001). A novel spike distance. Neural Comput. 13, 751-763.

Walz, H., Grewe, J. and Benda, J. (2014). Static frequency tuning properties account for changes in neural synchrony evoked by transient communication signals. J. Neurophysiol. 112, 752-765.

Woolley, S. M. N. and Moore, J. M. (2011). Coevolution in communication senders and receivers: vocal behavior and auditory processing in multiple songbird species. Ann. N. Y. Acad. Sci. 1225, 155-165.

Zakon, H. H. and Dunlap, K. D. (1999). Sex steroids and communication signals in electric fish: a tale of two species. Brain. Behav. Evol. 54, 61-9.

Zakon, H. H. and Smith, G. T. (2002). Weakly electric fish: behavior, neurobiology, and neuroendocrinology. Horm. brain Behav. 3, 349-375.

Zupanc, G. K. H. and Maler, L. (1993). Evoked chirping in the weakly electric fish Apteronotus leptorhynchus : a quantitative biophysical analysis. Can. J. Zool. 71, 23012310 .

Zupanc, G. K. H., Sirbulescu, R. F., Nichols, A. and Ilies, I. (2006). Electric interactions through chirping behavior in the weakly electric fish, Apteronotus leptorhynchus. J. Comp. Physiol. A 192, 159-173. 


\section{Chapter 4}

Prologue

In the previous two chapters I demonstrated the effects of signal structure and how they are used on chirp coding in A. leptorhynchus and A. albifrons. In both of these studies, stimuli were presented in a relatively simplified manner mimicking a pair of stationary fish. However, in natural environments, movement and the presence of additional fish contribute to creating a noisy and unpredictable sensory environment. The gregariousness of apteronotids varies between species (Stamper et al., 2010), thus the effects and intensity of conspecific noise also vary between species . All previous studies of chirp encoding have utilized the same simplified chirp stimulus regimes and it is unknown how apteronotids code chirps in these more complex and realistic environments. We expect that social species that have more complex communication signals to also encode variations in signal structure more accurately these. The social and chirping behavior of $A$. leptorhynchus, A. albifrons, and A. devenanzii provide a cross section of the diversity in apteronotids, making a comparative study between the three species ideal for determining the influence of context on chirp coding and whether sender-receiver matching adapts the sensory systems of gregarious species to efficiently accomplish this task.

In this chapter, I characterize chirp coding in A. devenanzii and assess chirp coding efficiency in all three species. I compare the coding performance in the presence and absence of social noise to determine if social species are specifically adapted to cope with it. I find that coding efficiency varies across these species, and that the most gregarious fish, $A$. devenanzii performs better than the others in all conditions. I also found that while base efficiency does vary across species, additional noise from conspecifics does not substantially decrease coding performance. This shows that these species share a mechanism to prevent social noise from deteriorating coding but that sociality may affect the overall accuracy of chirp coding. 
Note: This chapter will be submitted for publication as:

"Coding of naturalistic communication stimuli varies across three species of apteronotids", Kathryne Allen and Gary Marsat.

Kate Allen and Gary Marsat designed experiments and analysis, Kate Allen collected and analyzed all data. 


\section{Abstract}

The ability to detect the occurrence of communication signals is critical to the exchange of information. The presence of conspecific-generated noise poses a particular challenge to this efficient exchange. Animals must have sensory systems that are adapted to detect the characteristic features of conspecific signals, but in socially gregarious species, this means also being able to extract one of several communication signals with similar properties. In this study we use recordings from the hindbrains of three species of weakly electric fish with diverse life histories to show that the ability to detect and discriminate complex social signals is correlated with sociality. We present animals with stimuli that mimic naturalistic environments in which signals are perceived, including complex contrast changes from movement or the presence of multiple conspecifics. Our data suggest that differences across species in signal shape and neural properties result from adaptations to complex communication signals since more gregarious species encode the details of chirp structure with higher fidelity even when embedded in social noise. 


\section{Introduction}

Conspecific communication rarely occurs in noise-free conditions. Rather, information exchange happens in complex natural environments with multiple signals. Every organism must employ sensory adaptations for detecting faint or transient communication signals in the presence of their typical environmental noise as well as discriminating similar signals from each other (Wiley and Richards, 1978; Wiley and Richards, 1982). A major source of noise that is specific to gregarious species is that generated by conspecifics present in a group (Luther and Gentry, 2013). This is a particularly troublesome source of noise because in order to communicate sensory systems must be tuned to the natural properties of conspecific signals, but must also be able to filter and select only the features that are relevant in proper context (Bradbury and Vehrencamp, 2011). Many species face these problems, particularly those that sing in choruses, such as frogs or songbirds (Brumm and Slabbekoorn, 2005; Gerhardt and Huber, 2002; Wiley, 1994; Wollerman and Wiley, 2002) or those that live in large colonies, such as seabirds (Aubin and Jouventin, 2002). Adaptations to signal structure that facilitate communication in conspecific noise have been studied in a number of systems (Brumm and Slabbekoorn, 2005) as well as the effects of social noise on detection and discrimination levels (Lohr et al., 2003; Wollerman and Wiley, 2002). It is clear that social noise typically impairs the ability of receivers to accurately encode the signals of the sender. Likewise, the problems of sender-receiver matching and signal detection in noise have been thoroughly examined via neurophysiology (Langemann et al., 1998; Marsat et al., 2012; Neuhofer and Ronacher, 2012). However, the role of sender-receiver matching in light of increased sociality and conspecific generated noise is still unclear. In this study, we examine the neural responses to social signals in naturalistic noise and show that, despite the challenges associated with the task, the sensory system is highly performant at encoding stimuli with species-specific signal and noise structures. 
Apteronotid electric fish are a branch of the South American gymnotiform order of electric fish. The apteronotid family is diverse (Albert and Crampton, 2005), and widespread, spanning from Panama to Argentina (Mago-Leccia, 1994). Of these species, many of them are sympatric (Albert and Crampton, 2005; Kramer et al., 2013). This sympatry is a key strength for this study, as it suggests that while these species share habitat, prey, and predation pressures, a possible driver of sensory diversity is diversity in communication.

Apteronotid fish produce a continuous sinusoidal electric signal, or electric organ discharge (EOD) at very high frequencies (up to $2000+\mathrm{Hz}$ ) for both electrolocation and communication purposes. The primary modality for social communication in these fishes is the production of chirps: transient increases in EOD frequency accompanied by proportional decreases in EOD amplitude (Zakon and Smith, 2002). Chirps vary dramatically across apteronotids in their properties such as duration, frequency, and shape (Turner et al., 2007; Zakon and Smith, 2002). The behavioral significance of chirping and the circumstances of chirp production also vary across species (Dunlap and Larkins-Ford, 2003a; Hagedorn and Heiligenberg, 1985; Kolodziejski et al., 2007; Petzold et al., 2018).

The coding strategies used in response to chirps have been previously documented in both A. albifrons and A. leptorhynchus. In both of these species, beat frequency mediates how chirps are coded within the ELL (Allen and Marsat, 2018; Allen and Marsat, 2019). In both species, chirps produce distinct responses in the pyramidal neurons of the ELL. These pyramidal neurons fall into two major classes: ON-cells that respond to increases in signal amplitude and OFF-cells that respond to decreases (Saunders and Bastian, 1984). In A leptorhynchus small chirps on low frequency beats evoke a stereotyped burst response, especially among ON-cells, while high beat frequencies produce tonic increases in OFF-cell firing rate that are proportional 
to the duration of the chirp (Allen and Marsat, 2018; Marsat et al., 2009). Conversely, in $A$. albifrons chirps presented on low frequency beats produce inhibition in both cell types that lasts the duration of the chirp, but on high frequency beats chirps are coded with small increases in OFF-cell firing rate that quickly adapt and do not encode chirp duration (Allen and Marsat, 2019). As a result, A. leptorhynchus signals are best discriminated on high-frequency beats, but in A. albifrons performance is better for low frequencies.

In apteronotid communication, social noise is omnipresent since the interaction of two fishes' EODs cause amplitude modulations (Bullock, 1982). This amplitude modulation, or beat, is created by EODs of different frequencies constructively or destructively interacting as they move in and out of phase with each other. The beat frequency is equal to the difference in frequency of the two fish EODs. Since chirps are an increase in EOD frequency, the chirps are perceived as a transient change in the frequency of the beat. EOD waveform shape as well as the number of fish present will affect the complexity of the resulting beat (Fig 1A) (Petzold et al., 2016; Smith, 2013; Stamper et al., 2010). Additionally, the beat's strength (i.e. tertiary modulations also described as envelopes) increases and decreases as they swim closer or further (Stamper et al., 2013) (Fig 1B). As a result, detecting chirp occurrence or discriminating chirp features becomes more challenging as the number of fish in a group increase. While previous studies have examined the detailed mechanisms of chirp coding, all have relied on presenting chirps in a simplified manner with a two-fish beat (i.e. sinusoidal modulation of a single constant frequency (Benda et al., 2005; Benda et al., 2006; Metzen and Chacron, 2017; Vonderschen and Chacron, 2011)), presented at constant strength. While this protocol is useful for determining the basics of chirp reception in ideal conditions, the natural sensory environment is far more complex. The neural responses and encoding strategies used when the signal-to-noise ratio is low 
could be different. Thus, we investigated the impact of naturalistic noise conditions on the neural coding of communication signals.

For this study, we compared the neural coding of chirps in three species of fish, Apteronotus leptorhynchus, Apteronotus albifrons, and Adontosternarchus devenanzii. These species were selected for study because they offer a cross section of chirp frequency (Zhou and Smith, 2006) and chirp complexity (Turner et al., 2007). A. albifrons is largely territorial and chirps infrequently (Dunlap and Larkins-Ford, 2003a; Kolodziejski et al., 2007). $A$. leptorhynchus are known to form small, loose groups of 2-4 fish (Stamper et al., 2010) but exhibit frequent chirps tied to both courtship and agonistic encounters (Hagedorn and Heiligenberg, 1985; Henninger et al., 2018; Hupé and Lewis, 2008). A. devenanzii is very social and gregarious and produces frequent, highly complex chirps in a variety of social encounters (Zhou and Smith, 2006).

We hypothesize that in all species social noise will decrease the coding accuracy for chirps without changing the coding method or entirely losing the ability to signal the presence or identity of chirps. Furthermore, we hypothesize that the effect of social noise on chirp detection will be more pronounced in species that are less gregarious.

\section{Methods}

Animals:

All animals were housed according to WVU IACUC standards, protocol 151200009.2. All fish used were wild-caught, adult animals. A. devenanzii were provided by Dr. G. Troy Smith (Indiana University), while A. leptorhynchus and A. albifrons were provided by commercial fish 
suppliers. Fish were housed in small groups (1-14 individuals). Water in home tanks was maintained at 200-500 $\mu \mathrm{S}$ and 6.8-7.2 $\mathrm{pH}$.

\section{Electrophysiology}

Surgical techniques were similar to those previously described in (Allen and Marsat, 2018; Marsat et al., 2009). Fish were anaesthetized through surgery with Tricaine-S (MS-222, Western Chemicals Inc, $.25 \mathrm{~g} / \mathrm{L}$ water) and immobilized with an injection of Gallamine triethiodide $(.020 \mathrm{~g} / \mathrm{mL}$ saline, ApexBio). After surgery, fish were switched to anesthetic free water for respiration and allowed to recover for 20 minutes before recording. Recordings were made from pyramidal cells in the Lateral Segment (LS) of the ELL. Brain anatomy of all three species is similar, so major landmark blood vessels described in Maler et al., (1991) and electrode depth served as an adequate guide to locate LS pyramidal cells. In vivo recordings were made via metal filled extracellular electrodes (Frank and Becker, 1964) and amplified with an AM Systems amplifier (Model 1700). Data were recorded (Axon Digidata 1500 and Axoscope software) at a $20 \mathrm{kHz}$ sampling rate. ON and OFF cells were identified using known response properties, particularly responses to sinusoidal stimulation and spike triggered average waveforms calculated from responses to $0-60 \mathrm{~Hz}$ noise (Saunders and Bastian, 1984).

\section{Stimuli}

All stimuli were created in MatLab (Mathworks, Inc.) and sampled at $20 \mathrm{kHz}$. Stimulation was provided by a direct modulation of the amplitude (AM) of a carrier artificial EOD phase locked to the fish's own (Benda et al., 2005; Krahe et al., 2008; Marsat et al., 2009) and allows precise control over the stimulus AM. Baseline EOD was recorded via electrodes near the head and tail of the fish. Each EOD cycle triggered a sine wave generator (Rigol 
DG1022A) to generate one cycle of a sine wave matched to the animal's own. This signal was then multiplied using a custom-built signal multiplier (courtesy of the Fortune Laboratory, New Jersey Institute of Technology) by the AM stimulus to create the desired modulation of the electric field. Stimuli were played through a custom-built stimulus isolator into the experimental tank via either two $30.5 \mathrm{~cm}$ carbon electrodes arranged parallel to the fish's longitudinal axis (global stimulation) or two silver chloridized point electrodes $1 \mathrm{~cm}$ apart from each other positioned near the receptive field on the fish's skin (local stimulation). The stimulus strength was adjusted to provide $\sim 20 \%$ contrast (the difference between the maximum and minimum of amplitude modulation divided by the baseline EOD).

Random amplitude modulation (RAM) stimuli consisted of 30 seconds of random noise filtered using a broadband $(0-60 \mathrm{~Hz})$ Butterworth filter. The stimulus was played for three repetitions in both global and local stimulation configurations. Sinusoidal amplitude modulation (SAM) stimuli were 2 second long stimuli modulated at 2, 5, 15, 30, 60, and $90 \mathrm{~Hz}$ with 2 seconds rest between each frequency, repeated at least three times. Step stimulations were $100 \mathrm{~ms}$ long $20 \%$ increases or decreases in amplitude repeated for 30 seconds.

Our chirp stimuli's AM were generated by creating EOD signals with the desired characteristics and extracting the AM waveform to be stored for use during the experiments. Chirp stimuli were created by using recorded EOD samples (courtesy of Dr. G. Troy Smith, Electric Fish Signal Library) to create template EOD waveform shape for each species thereby not simplifying the EOD to a pure sinewave. The EOD frequency of the "focal" signal chosen in this process is inconsequential since the only the AM waveform is saved. For each species, a focal $800 \mathrm{~Hz}$ EOD was created using this template waveform. For the dyad condition, a second EOD of either 810 or $900 \mathrm{~Hz}$ was created. This EOD was then multiplied by a low-pass filtered 
random amplitude modulation $(<2 \mathrm{~Hz})$. Conspecific chirps were each embedded in this modulated EOD by decreasing the EOD period by the inverse of the desired frequency increase for each cycle in the duration of the chirp. Chirps were added semi-randomly with a total of 76 chirps per 2 minute long stimulus ensuring that a range of stimulus amplitudes and chirps occurring at many phases of the beat cycle were produced. This chirper EOD was added to the original $800 \mathrm{~Hz}$ EOD. The AM of this stimulus was extracted by rectifying and low-pass filtering the combined EOD signal using MatLab's spline function. Note that extracting the AM modulation signal using a Hilbert transform gave similar results. This process was repeated for each beat frequency $(+10 \mathrm{~Hz}$ and $+100 \mathrm{~Hz})$ and conspecific chirp used, resulting in eight total "Dyad" stimuli. A full description of all chirps and their properties used for each species can be found in Table 1. Each 2 minute long AM signal was delivered during the experiments as described above.

"Group" (focal fish, chirper, and two observers) stimuli were created in a similar manner. After adding the chirper EOD to the $800 \mathrm{~Hz}$ focal EOD, two additional low-frequency amplitude modulated EODs were added to simulate the presence of additional fish in the interaction. The frequencies of the added EODs were $830 \mathrm{~Hz}$, and either $810 \mathrm{~Hz}$ or $900 \mathrm{~Hz}$, opposite of the frequency of the chirper EOD. Thus, the frequency content of each resulting stimulus was $10 \mathrm{~Hz}$, $30 \mathrm{~Hz}$, and $100 \mathrm{~Hz}$ of $\mathrm{AM}$ and approximately $2 \mathrm{~Hz}$ ongoing envelope modulation. The AMs of these additional EOD stimuli were roughly complementary to each other so that resultant signal would maintain a relatively constant overall strength. The result was 8 total "Group" stimuli. All chirp stimuli were only played in the global configuration. 
For all analyses spike trains were first binarized using a bin width of $0.5 \mathrm{~ms}$. All analyses described here were performed on these binary sequences in MatLab. Statistical analyses (ANOVA, t-test) were performed as stated using MatLab's statistical analysis toolbox.

\section{Chirp Analysis}

For detection analysis, spike distance metrics were used to calculate distance between spike trains of chirp and beat responses. The combined responses of ON and OFF cells were

used. Binarized spike trains were first convolved with a filter of $f(t)=t * \exp \left(-\frac{2.45 t}{\tau}\right)$ with a width of $30 \mathrm{~ms}$ at half maximum $(\tau)$. A window of each filtered spike train around the chirp $\left(R_{c}(t)\right)$ of was extracted from the spike train and compared to a window of beat of the same size $\left(\mathrm{R}_{\mathrm{b}}(\mathrm{t})\right)$. Windows were adjusted to accommodate the longest chirps of each species $(A$. leptorhynchus: 50ms, A. albifrons: 200ms, A. devenanzii: $400 \mathrm{~ms}$ ). These beat and chirp responses were then assigned weights using Kullback-Liebler divergence to quantify how much the two set of responses overlap in Euclidian space. For a randomly chosen subset of the chirp and beat response pairs, a probability distribution of firing rates in each dimension (a given neuron's response at a given time point constitutes one dimension) was generated (chirp: $P(c)$ and beat:P(b)) and the divergence between distributions was calculated for each dimension as:

$$
D_{K L}=\sum P(c) *\left(\log P_{c}-\log P_{b}\right)
$$

The resulting $D_{K L}$ values could be compared as the synaptic weight onto a decoder that would differ for each input neuron but also change as a function of time. While systematic changes in synaptic weight through time (e.g. due to facilitation or depression) are conceivable for a biological decoding system, non-systematic changes are not biologically plausible. For this 
reason we averaged $D_{K L}$ values across time for a given neuron thereby loosely mimicking a synaptic weight that is fixed across time for a given neuron. The $D_{K L}$ values were then normalized to a mean of 1 . These divergence values were used to assign a weight to individual neurons in the subsequent analysis of detection and discrimination.

To analyze distances between $R_{c}(t)$ and $R_{b}(t)$, the remaining subset of responses, those not used to calculate $D_{K L}$, were multiplied by $D_{K L}$. The Euclidean distance between these responses was calculated as

$$
D_{c b}=\sqrt{\sum\left(\left(D_{K L c} R_{c}\right)_{n}-\left(D_{K L b} R_{b}\right)_{n}\right)^{2}}
$$

Where $n$ is the number $x^{*} y$ of dimensions used in the analysis (for $x$ neurons and $y$ time points). Discrimination analysis was conducted in the same manner, but rather than comparing chirp vs. beat, different chirps were compared $\left(R_{c x}(t)\right.$ and $\left.R_{c y}(t)\right)$ for each possible chirp combination.

The probability distributions of the values in these arrays $\left(P\left(D_{c b}\right), P\left(D_{b b}\right), P\left(D_{c x x}\right)\right)$, and $\left.P\left(D_{c x y}\right)\right)$ were then used to determine error levels for an ideal observer determining detection or discrimination. ROC curves were calculated by varying a threshold distance $(T)$ to separate chirp and beat responses. For each threshold the probability of detection $(P D)$ was calculated as the sum of $\left(P\left(D_{c b}>T\right)\right)$, and the probability of false alarm $(P F)$ as the sum of $\left(P\left(D_{b b}<T\right)\right)$. The error level for each threshold value is $E=P F / 2+(1-P D) / 2$. The values shown in Figure 3 are the minimum calculated values of $E$ as increasing numbers of spike trains are included in the calculation. 


\section{Results:}

\section{Chirp coding across apteronotids}

To characterize the responses of ELL pyramidal neurons to various types of chirps, a selection of four species-typical conspecific chirps were played to each fish. A full description of chirps used is located in Table 1. Chirp properties were based on natural ranges as reported by previous studies of each species' behavior (Dunlap and Larkins-Ford, 2003b; Zhou and Smith, 2006; Zupanc and Maler, 1993). To characterize as wide a range of neural responses as possible, we selected chirps that represented the reported minimum and maximum limits of chirp ranges in terms of duration, frequency increase, and in the case of $A$. devenanzii, number of frequency peaks in the chirp.

Our data confirm the previously reported firing patterns in A. leptorhynchus and $A$. albifrons, as well as characterize the coding strategies used by A. devenanzii. Shown in Figure 2 are representative OFF-cell responses to the longest of chirps used in each species presented on $10 \mathrm{~Hz}$ and $100 \mathrm{~Hz}$ beats. We find that $A$. devenanzii exhibit a pattern of chirp coding more similar to that of $A$. albifrons than A. leptorhynchus with the same chirp producing inhibition on low frequencies, but excitation on high frequencies (Fig 2A). However, unlike A. albifrons, rather than the quickly adapting excitation observed on high frequencies, A. devenanzii pyramidal neurons produce multiple peaks in firing rate that correspond to the multiple frequency peaks within the chirp, as well as inhibition that signals the end of the chirp (Fig 2B). This suggests that this species is capable of accurately encoding details about chirp structures on all frequencies. 
As described above, chirps in all three species produce distinct responses among ELL neurons, however previous studies have only quantified chirps when presented in a very simplified manner (a beat consisting in a sinusoidal AM of fixed contrast (Benda et al., 2006; Marsat et al., 2009; Vonderschen and Chacron, 2011)). In natural interactions, fish are generally moving rather than stationary, increasing and decreasing the contrast of the amplitude modulations produced by chirps. To simulate the effects of these changes in contrast on chirp reception we played chirp stimuli simulating a single chirping fish of either $10 \mathrm{~Hz}$ or $100 \mathrm{~Hz}$ EOD frequency above the focal fish, modulated by low frequency envelopes (Dyad condition). Simply including these contrast changes produces chirp responses that are less obvious and distinct compared to the results from previous studies (Fig 2 B). Longer chirps, however, produce a more obvious, slight increase in firing rate among OFF-cells (Fig 2A). This was not as distinctive as the response produced by chirps presented on a $100 \mathrm{~Hz}$ beat (Fig 2B)

We performed a spike metric distance analysis to determine if there were differences in the accuracy with which these three species were able to detect chirps. The results are displayed as error level ( 0.5 indicating chance level detection, 0 being perfect detection) as a function of the number of neural inputs pooled in the analysis. A steeper decline in the curve indicates that each neuron is contributing more information about the presence of a chirp. Across all three species, our results suggest that with a large enough population of neurons, detection of all conspecific chirp types is possible (Fig 3), this is unsurprising, given that this task is something these animals encounter regularly. What is unexpected, is that with this analysis, we do not see the beat specific differences that have been observed with more conservative measures (Allen and Marsat, 2018; Allen and Marsat, 2019; Marsat and Maler, 2010). This result may be due to differences in analysis since we use a metric that weights information-rich responses more 
heavily and noisy responses less (see Methods and Discussion). Consistently across both beat frequencies, $A$. devenanzii perform very well, reaching $<.10$ error with a comparatively small number of neurons (Figure 3).

Our data also show that the ability to discriminate between chirps from ELL neural responses varies across the three species. Similarly, we were surprised to not find a large difference as the result of beat frequency in any of the three species. We also found, somewhat counterintuitively that chirp discrimination was more accurate than mean detection (Fig 4A). This may be the result of using chirps that represent opposite ends of species typical ranges, and are thus most easily distinguishable. This, paired with the variability of the background beat producing highly variable beat responses, may produce chirp responses that are more consistent and easier to distinguish from each other than individual chirp responses compared against variable beat responses. Like our measure of detection, we find that $A$. devenanzii show a steep decrease in error rates, compared to both A. leptorhynchus and A. albifrons (Fig 4)

\section{Chirp coding in social Noise}

To determine if there are differences across species that would facilitate the detection of signals in social setting with multiple fish, we played the same chirp stimuli described above, but further simulated the presence of two additional "observer" fish that do not chirp (Group Condition). These additional signals, like the chirp-containing signal were modulated in contrast, mimicking movement. The responses to these noisy chirps are shown in Figure 5. Looking at the raster plots of chirp responses (Fig 5A), the patterns of neural responses are similar to those observed in the dyad condition, with chirps causing inhibition on low-frequency beats and excitation on high frequency beats. While the general pattern of activity is similar, the strength of 
chirp responses is much weaker. The firing rate changes in response to chirps are still present, but much subtler (Fig 5B).

We found that contrary to the expectations based on these subtle responses, the presence of additional fish did not have a large qualitative effect on coding efficiency. In most instances the accuracy of chirp detection was only slightly worse than in the dyad condition (Fig 6). These counterintuitive findings may be explained by the effect of adding social beats on the variability of responses. If the variability of chirp responses increase (as expected from the addition of social noise) but the variability in background responses decreases, the detection performance can remain relatively unaffected. A decrease in variability of background responses is possible (compare grey curves in Figures 2B and 5B) if noisy responses are relatively uncorrelated across neurons, allowing the variability to be averaged out of the population response.

For our measure of discrimination as well, there was not a large decrease in accuracy for any species, but the trends observed in our dyad conditions were consistent, with A. leptorynchus and A. albifrons exhibiting comparatively poor coding accuracy, while $A$. devenanzii demonstrated higher levels of accuracy (Fig 7A).

\section{Quantification of detection accuracy}

A system that encodes a high amount of non-redundant information in each neural response would show a large drop in error level as the population size increases from single cells to a few neurons. Therefore, we quantified the encoding accuracy and efficiency, based on the steepness of the curves in Figures 3, 4, 5 and 6, by calculating a ratio of the error levels at two points: population sizes of 1 neuron and 10 neurons. In both the dyad and group conditions $A$. devenanzii responses allowed the most efficient detection of chirp occurrence while $A$. 
leptorhynchus performed the worst (Figure 8A) (ANOVA, $\mathrm{p}=.006$ ). The same is also true of discrimination (Figure 8B) (ANOVA, $\mathrm{p}<.0001$ ). For both detection and discrimination $A$. devenanzii also performed better than the other two species in group conditions (ANOVA: detection- $\mathrm{p}<.0001$, discrimination- $\mathrm{p}<.0001)$. Contrary to our expectations, adding social noise had no effect or even slightly improved detection and discrimination accuracy (Detection $\mathrm{t}$ test: A. leptorhynchus $\mathrm{p}=.0038$, A. devenanzii $\mathrm{p}=0.9305$, A. alibifrons $\mathrm{p}=0.5834$; Discrimination $\mathrm{t}$ test: A. leptorhynchus $\mathrm{p}=0.3900$, A. devenanzii $\mathrm{p}=0.9328$, A. alibifrons $\mathrm{p}=0.0875$ )

Our spike distance metric used for the detection/discrimination analysis is based on a Euclidian distance where each neural response is represented in different dimensions. For this reason, ON and OFF responses could be pooled in the analysis. The more conservative analysis used in preceding studies (Allen and Marsat, 2018; Allen and Marsat, 2019; Marsat and Pollack, 2010) could not do so because the mirror-image responses would have been summed and have cancelled each other. These studies pointed out that ON- and OFF-cells performed differently in how their responses could support detection and discrimination. We questioned if a similar difference in ON- and OFF-cell coding accuracy could be observed when stimulated with our dyad/group stimuli. We also wondered if our analysis would reveal differences in performance when the cell types are analyzed separately versus jointly. Although small differences in coding efficiency could be observed between ON- and OFF-cells for all species and condition, only detection in group conditions for A. devenanzii was significantly more accurate in OFF-cells (Fig $9 ; \mathrm{t}$ test, $\mathrm{p}=0.0165)$. Furthermore, the coding efficiency calculated using both ON and OFF-cells was generally not better than the best of the individual populations (see Fig 9 and legend for full statistics) suggesting that there is no synergistic effect. 
Another difference in our protocol compared to past experiments on this topic is that our stimulus constantly varied in contrast. This stimulus reflects more naturalistic conditions since fish experience chirps at various intensities. It also replicates a more realistic decoding task where decoding networks must cope with this variability and thus responses to chirps with variable intensities must be classified as similar despite the difference in response patterns caused by the variable intensity. Despite this difficulty, our analysis shows that neural responses are more similar across presentations of a given chirps than across different chirps since the decoding analysis was able to perform detection/discrimination with low error rates (Figs 3-4, 67). Our stimuli also allowed us to ask how coding performance changed with chirp intensity. To answer this question, we analyzed the responses to chirps occurring during portion of beats with low, medium, or high contrast separately. Focusing on the detection task (discrimination showed a similar trend; data not shown), we found as expected that chirps of low intensity were less accurately encoded (Fig 10). The higher coding efficiency of $A$. devenanzii neural responses we describe above is even more evident for chirps of low intensity (ANOVA: Dyad 10hz-p=0.0025, Dyad 100Hz-p=.0004). This effect strengthens our conclusion that A. devenanzii's neural response are more accurate at encoding chirp stimuli. Most importantly, the effect of social noise was again negligible on the encoding performance.

\section{Responses to simple chirps also varies across species}

To determine if our observed differences in coding accuracy were due to differences in neural processing, or just the result of signal structure differences, we also played two $A$. leptorhynchus small chirps, presented on a simplified, constant strength $10 \mathrm{~Hz}$ beat, to all three species. These stimuli match those used in previous studies of A. leptorhynchus chirp coding. We find that $A$. leptorhynchus are best at detecting their own chirps in these conditions, and 
surprisingly, considering the preceding data, A. devenanzii were most inefficient at detection small chirps (Fig 11A). This suggest that while A. devenanzii chirp structure may be particularly conspicuous, there are also differences in chirp encoding, that make them adept at coding these long chirps over heterospecific chirps. A. devenanzii responses also produce poor discrimination of A. leptorhynchus chirps, while A. albifrons and A. leptorhynchus may be able to discriminate small chirps (in this case presented on fixed phases), it is still error prone compared to other chirp types (Allen and Marsat, 2019; Marsat and Maler, 2010) even with our liberal analysis (Fig 11B. 


\section{Discussion}

\section{Summary of findings}

Using naturalistic stimuli that vary in intensity and that contain the background signal of additional fish ("social noise") we show that neural encoding of chirps is strikingly resilient to conspecific noise. Neural responses were qualitatively similar to the characterization in previous reports (Allen and Marsat, 2019; Marsat and Pollack, 2010). A. devenanzii, that had not been studied in the past, encode chirps in graded heterogeneous increases in firing rate reflecting the frequency modulation profile (accompanied by an inversely correlated amplitude modulation profile) of the chirp. Our analysis pooled the responses of ON- and OFF-cells as has been suggested to occur in the cell's TS targets. The trends described were present, whether using both cell types or each type individually despite small differences in coding performance (see below). Comparing the encoding performance of the three species, $A$. devenanzii was markedly better than the other two species, although this is in part due to differences in the structure of the signals. Together, these data show that the sensory system of apteronotids is capable of accurately detecting and discriminating chirps even in the presence of strong social noise, and that some species have a communication system better suited for communicating in difficult conditions.

\section{Species differences in chirp coding}

Chirp coding strategies are generally similar across species consisting in graded heterogeneous increases and decreases in firing mirroring the chirp's modulation profile. Nevertheless, a number of noteworthy differences are present. In A. devenanzii, chirps presented on low frequencies generally produce inhibition lasting the duration of the chirp, much like what is observed to produce relatively accurate chirp discriminations in A. albifrons (Allen and 
Marsat, 2019). On high frequency beats, chirps evoke an increase in firing that that is also proportional to the length of the chirp, more similar to what is observed in A. leptorhynchus, which also allows for accurate chirp discrimination. Therefore, differences in adaptation rate explain some differences in the responses, while difference in chirp structure such as their long multipeaked nature (Zhou and Smith, 2006) play a key role in explaining other differences. This particular combination of signal structure and neural properties enables accurate discrimination of chirps in all social contexts, whereas both A. albifrons and A. leptorhynchus performance was either lower (present data) or accurate only for a given beat context (Allen and Marsat, 2019). This may be reflective of $A$. devenanzii's ethology, as they are generally more social than $A$. leptorhynchus and A. albifrons. Additionally, EOD frequency is less sexually dimorphic in the species (Zhou and Smith, 2006). This means that chirps of all types, even the multi-peaked chirps produced mainly by male fish, are more likely to be encountered on a wide variety of beat frequencies, thus reducing the need for context specific feature extraction and increasing the need for chirp assessment in all beat contexts.

Apteronotids at the cocktail party

Contrary to our hypothesis, the addition of conspecific generated social noise did little to hamper coding as compared to a less noisy, but realistic condition mimicking a dyadic interaction. While this result is unexpected, it is not necessarily surprising. Evidence from both auditory and somatosensory systems suggest that some amount of noise, or stochastic resonance, can actually improve detection thresholds (Hänggi, 2002; Wells et al., 2005; Zeng et al., 2000) and in an animal uniquely specialized for detection of faint electric signals, it is not shocking that they are able to perform this task well. 
Our goal was to test the ability to encode chirp in conditions more difficult and closer to realistic than the paradigm used in previous studies where signal intensity was constant, not weak, and contained no noise. When analyzed with the methods used in these studies, the responses to our more challenging stimuli appeared unable to support detection and discrimination of the signals as error rates where close to chance-level for nearly all signals and species. This more conservative method, based on the spike metric distance introduced by van Rossum (van Rossum, 2001), sums the responses from the different neurons whereas the present method keeps the responses from different neurons as separate dimension, thereby allowing a spike from one neuron (e.g. an OFF-cell) to have a different meaning than a spike from another neurons (e.g. ON-cell). Most importantly, the contribution from each neuron is weighted proportionally to how informative its responses are. Neurons that do not respond well to chirps can be mostly ignored, thereby preventing them from adding noise to the population response. This decoding procedure is not unrealistic, since it is common for synaptic inputs from different neurons to be weighted differently. The ELL contains a diversity of pyramidal cells and while we focused our recordings on superficial and intermediate cells of the LS, these cells vary in receptive fields, firing rate, tuning and more. It is plausible that a decoder network could be preferentially connected to a subset of these cells. This enhanced decoding method is bound to extract more information from the population response than the conservative method used in previous publications. This type of analysis does not imply that the amount of information extracted by our decoder is in fact used by the system, but rather that the information is present in the spike train and is an estimation of what the decoder could extract.

The use of this enhanced method, or the more challenging condition that our stimulus mimics, can explain the differences between this and previous studies regarding which beat 
frequency, cell type or species display better neural detection/discrimination accuracy. It does not mean that past results are invalid or that current results overestimate the measured quantities. The measures and the perspectives should be seen as complementary, one providing a higherbound estimate of accuracy, the other a lower-bound estimate. The actual amount of information used to generate behavior or that is decoded at a particular step can only be quantified precisely by characterizing these levels directly. The fact that we previously demonstrated a link between the results of our analysis and behavioral performance (Allen and Marsat, 2018) demonstrate that these types of analysis provide a perspective on how sensory information is processed. Similar experiments testing behavior or higher brain areas should be performed to confirm that the phenomena described here relate directly to the overall performance of the system.

\section{Evidence for sender-receiver matching}

In all conditions, both low and high noise, coding accuracy did vary across the three species. Our data indicate that the most social species was indeed best at both detecting and discriminating among chirps in both conditions. This is likely a product of changes in neural properties such as frequency tuning or adaptation rate (supplemental figure S1), as well as chirps that are adapted to be longer, conspicuous, and more variable in structure (Zhou and Smith, 2006). This matching between a signal rich in information and a sensory system adapted to extract this information provides evidence to support the social-complexity theory of communication. According to this theory, increased social complexity correlates with increases in communication complexity (Blumstein and Armitage, 1997). A natural consequence of this evolutionary process would be that sensory system must be able to encode the subtleties of the complex communication signals in order to extract the information the variations carry. In the 
present case, the highly variable chirp structure in $A$. devenanzii is met with a nervous system capable of accurate encoding of these signals.

\section{Conclusion}

Studying the neural mechanisms that enable the extraction of information from complex natural scenes is a common goal sensory system research. In studying communication particularly, a number of studies have revealed specific adaptations that allow animals to accurately detect social signals in noise. In the auditory system, several mechanisms have been proposed to accomplish signal extraction, such as high-order neurons that respond selectively to spectral envelope shapes that are integrated over time from lower-order neurons (Moore et al., 2013). This integration of low-order responses may also be responsible for the suppression of responses to noise, as long-term ongoing sounds are time averaged together to create an acoustic "texture" (McDermott et al., 2013). This selectivity for more derived signal features may enable transient events, like communication, to better stand out against this background. Interestingly, our results provide evidence that there are mechanisms even at a comparatively low-order stage of sensory processing that enables accurate feature extraction in noise. While we did not directly investigate the mechanisms that enable chirp detection in noise, our results show that even in uncommon sensory systems like electroreception, there is sender-receiver matching that allows signal detection in the challenging environments in which they evolved. 


\section{Works Cited}

Albert, J. S. and Crampton, W. G. R. (2005). Diversity and Phylogeny of Neotropical Electric Fishes (Gymnotiformes). In Electroreception (ed. Bullock, T. H.), Hopkins, C. D.), Popper, A. N.), and Fay, R. R.), pp. 360-409. New York, NY: Springer New York.

Allen, K. M. and Marsat, G. (2018). Task-specific sensory coding strategies matched to detection and discrimination behaviors in Apteronotus leptorhynchus. J. Exp. Biol. 221,.

Allen, K. M. and Marsat, G. (2019). Neural processing of communication signals: The extent of senderreceiver matching varies across species of Apteronotus. eneuro ENEURO.0392-18.2019.

Aubin, T. and Jouventin, P. (2002). How to vocally identify kin in a crowd: the penguin model. Adv. Study Behav. 31, 243-277.

Benda, J., Longtin, A. and Maler, L. (2005). Spike-frequency adaptation separates transient communication signals from background oscillations. J. Neurosci. 25, 2312-21.

Benda, J., Longtin, A. and Maler, L. (2006). A synchronization-desynchronization code for natural communication signals. Neuron 52, 347-58.

Blumstein, D. T. and Armitage, K. B. (1997). Does Sociality Drive The Evolution Of Communicative Complexity? A Comparative Test With Ground-Dwelling Sciurid Alarm Calls. Am. Nat. 150, 179-200.

Bradbury, J. W. and Vehrencamp, S. L. (2011). Principles of Animal Communication. 2nd ed. Sunderland, Mass.: Sunderland: Sinauer Associates.

Brumm, H. and Slabbekoorn, H. (2005). Acoustic Communication in Noise. Adv. Study Behav.

Bullock, T. H. (1982). Electroreception. Annu. Rev. Neurosci. 5, 121-170.

Dunlap, K. D. and Larkins-Ford, J. (2003a). Production of aggressive electrocommunication signals to progressively realistic social stimuli in male Apteronotus leptorhynchus. Ethology 109, 243-258.

Dunlap, K. D. and Larkins-Ford, J. (2003b). Diversity in the structure of electrocommunication signals within a genus of electric fish, Apteronotus. J. Comp. Physiol. A 189, 153-161.

Frank, K. and Becker, M. C. (1964). Microelectrodes for recording and stimulation. In Physical techniques in biological research (ed. Nastuk, W. L.), pp. 23-84. New York: Academic Press.

Gerhardt, H. C. and Huber, F. (2002). Acoustic Communication in Insects and Anurans: Common Problems and Diverse Solutions. University of Chicago Press.

Hagedorn, M. and Heiligenberg, W. (1985). Court and spark: electric signals in the courtship and mating of gymnotoid fish. Anim. Behav. 33, 254-265.

Hänggi, P. (2002). Stochastic resonance in biology how noise can enhance detection of weak signals and help improve biological information processing. ChemPhysChem 3, 285-290.

Henninger, J., Krahe, R., Kirschbaum, F., Grewe, J. and Benda, J. (2018). Statistics of natural communication signals observed in the wild identify important yet neglected stimulus regimes in weakly electric fish. J. Neurosci. 0350-18.

Hupé, G. J. and Lewis, J. E. (2008). Electrocommunication signals in free swimming brown ghost knifefish, Apteronotus leptorhynchus. J. Exp. Biol. 211, 1657-1667. 
Kolodziejski, J., Sanford, S. E. and Smith, G. T. (2007). Stimulus frequency differentially affects chirping in two species of weakly electric fish: implications for the evolution of signal structure and function. J. Exp. Biol. 210, 2501-2509.

Krahe, R., Bastian, J. and Chacron, M. J. (2008). Temporal processing across multiple topographic maps in the electrosensory system. J. Neurophysiol. 100, 852-67.

Kramer, B., Kirschbaum, F. and Markl, H. (2013). Species Specificity of Electric Organ Discharges in A Sympatric Group of Gymnotoid Fish From Manaus (Amazonas). In Sensory Physiology of Aquatic Lower Vertebrates, pp. 195-219.

Langemann, U., Gauger, B. and Klump, G. M. (1998). Auditory sensitivity in the great tit: perception of signals in the presence and absence of noise. Anim. Behav. 56, 763-769.

Lohr, B., Wright, T. F. and Dooling, R. J. (2003). Detection and discrimination of natural calls in masking noise by birds: Estimating the active space of a signal. Anim. Behav. 65, 763-777.

Luther, D. and Gentry, K. (2013). Sources of background noise and their influence on vertebrate acoustic communication. Behaviour 150, 1045-1068.

Mago-Leccia, F. (1994). Electric fishes of the continental waters of America. Biblioteca de la Academia de Ciencias Fisicas, Matematicas, y Naturals, Caracas. Venezuela 29, 1-206.

Maler, L., Sas, E., Johnston, S. and Ellis, W. (1991). An atlas of the brain of the electric fish Apteronotus leptorhynchus. J. Chem. Neuroanat. 4, 1-38.

Marsat, G. and Maler, L. (2010). Neural heterogeneity and efficient population codes for communication signals. J. Neurophysiol. 104, 2543-55.

Marsat, G. and Pollack, G. S. (2010). The structure and size of sensory bursts encode stimulus information but only size affects behavior. J. Comp. Physiol. A Neuroethol. Sensory, Neural, Behav. Physiol. 196, 315-320.

Marsat, G., Proville, R. D. and Maler, L. (2009). Transient signals trigger synchronous bursts in an identified population of neurons. J. Neurophysiol. 102, 714-23.

Marsat, G., Longtin, A. and Maler, L. (2012). Cellular and circuit properties supporting different sensory coding strategies in electric fish and other systems. Curr. Opin. Neurobiol. 22, 686-692.

McDermott, J. H., Schemitsch, M. and Simoncelli, E. P. (2013). Summary statistics in auditory perception. Nat. Neurosci. 16, 493.

Metzen, M. G. and Chacron, M. J. (2017). Stimulus background influences phase invariant coding by correlated neural activity. Elife 6,.

Moore, R. C., Lee, T. and Theunissen, F. E. (2013). Noise-invariant Neurons in the Avian Auditory Cortex: Hearing the Song in Noise. PLoS Comput. Biol. 9,.

Neuhofer, D. and Ronacher, B. (2012). Influence of Different Envelope Maskers on Signal Recognition and Neuronal Representation in the Auditory System of a Grasshopper. PLoS One 7, e34384.

Petzold, J. M., Marsat, G. and Smith, G. T. (2016). Co-adaptation of electric organ discharges and chirps in South American ghost knifefishes (Apteronotidae). J. Physiol.

Petzold, J. M., Alves-Gomes, J. A. and Smith, G. T. (2018). Chirping and asymmetric jamming avoidance 
responses in the electric fish \&lt;em\&gt;Distocyclus conirostris\&lt;/em\&gt; J. Exp. Biol. 221, jeb178913.

Saunders, J. and Bastian, J. (1984). The physiology and morphology of two types of electrosensory neurons in the weakly electric fish Apteronotus leptorhynchus. J. Comp. Physiol. A 154, 199-209.

Smith, G. T. (2013). Evolution and hormonal regulation of sex differences in the electrocommunication behavior of ghost knifefishes (Apteronotidae). J. Exp. Biol. 216, 2421 LP-2433.

Stamper, S. A., Carrera-G, E., Tan, E. W., Fugère, V., Krahe, R. and Fortune, E. S. (2010). Species differences in group size and electrosensory interference in weakly electric fishes: Implications for electrosensory processing. Behav. Brain Res. 207, 368-376.

Stamper, S. A., Fortune, E. S. and Chacron, M. J. (2013). Perception and coding of envelopes in weakly electric fishes. J. Exp. Biol. 216, 2393 LP-2402.

Turner, C. R., Derylo, M., de Santana, C. D., Alves-Gomes, J. a and Smith, G. T. (2007). Phylogenetic comparative analysis of electric communication signals in ghost knifefishes (Gymnotiformes: Apteronotidae). J. Exp. bBology 210, 4104-4122.

van Rossum, M. C. (2001). A novel spike distance. Neural Comput. 13, 751-763.

Vonderschen, K. and Chacron, M. J. (2011). Sparse and dense coding of natural stimuli by distinct midbrain neuron subpopulations in weakly electric fish. J. Neurophysiol. 106, 3102-18.

Wells, C., Ward, L. M., Chua, R. and Inglis, J. T. (2005). Touch Noise Increases Vibrotactile Sensitivity in Old and Young. Psychol. Sci. 16, 313-320.

Wiley, R. H. (1994). Errors, exaggeration, and deception in animal communication. Behav. Mech. Evol. Ecol. 157-189.

Wiley, R. H. and Richards, D. G. (1978). Behavioral Ecology and Sociobiology Physical Constraints on Acoustic Communication in the Atmosphere: Implications for the Evolution of Animal Vocalizations. Behav. Ecol. Sociobiol 3, 69-94.

Wiley, R. H. and Richards, D. G. (1982). Adaptations for acoustic communication in birds: sound transmission and signal detection. Acoust. Commun. birds 1, 131-181.

Wollerman, L. and Wiley, R. H. (2002). Background noise from a natural chorus alters female discrimination of male calls in a Neotropical frog. Anim. Behav. 63, 15-22.

Zakon, H. H. and Smith, G. T. (2002). Weakly electric fish: behavior, neurobiology, and neuroendocrinology. Horm. brain Behav. 3, 349-375.

Zeng, F.-G., Fu, Q.-J. and Morse, R. (2000). Human hearing enhanced by noise11Published on the World Wide Web on 23 May 2000. Brain Res. 869, 251-255.

Zhou, M. and Smith, G. T. (2006). Structure and sexual dimorphism of the electrocommunication signals of the weakly electric fish, Adontosternarchus devenanzii. J. Exp. Biol. 209, 4809-4818.

Zupanc, G. K. H. and Maler, L. (1993). Evoked chirping in the weakly electric fish Apteronotus leptorhynchus : a quantitative biophysical analysis. Can. J. Zool. 71, 2301-2310. 


\section{Figures and Tables}

Table 1:

A summary of all chirps used. For each species, a selection of chirps from reported ranges were used, two from the smallest end of the range, and two from the largest. The category of other refers to properties of the chirp not related to length or frequency, such as the shape of frequency rise and decay, or number of peaks in frequency.

\begin{tabular}{|c|c|c|c|}
\hline Chirp & Frequency $(\mathrm{Hz})$ & Length (ms) & Other \\
\hline \multicolumn{4}{|c|}{ A. leptorhynchus } \\
\hline 1 & 60 & 10 & Gaussian \\
\hline 2 & 122 & 15 & Gaussian \\
\hline 3 & 300 & 15 & Gaussian \\
\hline 4 & 900 & 45 & Gaussian \\
\hline \multicolumn{4}{|c|}{ A. Albifrons } \\
\hline 1 & 50 & 50 & Alpha \\
\hline 2 & 100 & 50 & Alpha \\
\hline 3 & 200 & 100 & Alpha \\
\hline 4 & 350 & 200 & Alpha \\
\hline \multicolumn{4}{|c|}{ A. Devenanzii } \\
\hline 1 & 100 & 40 & Alpha \\
\hline 2 & 100 & 100 & 2 peaks \\
\hline 3 & 400 & 200 & Alpha \\
\hline 4 & 400 & 400 & 8 peaks \\
\hline
\end{tabular}



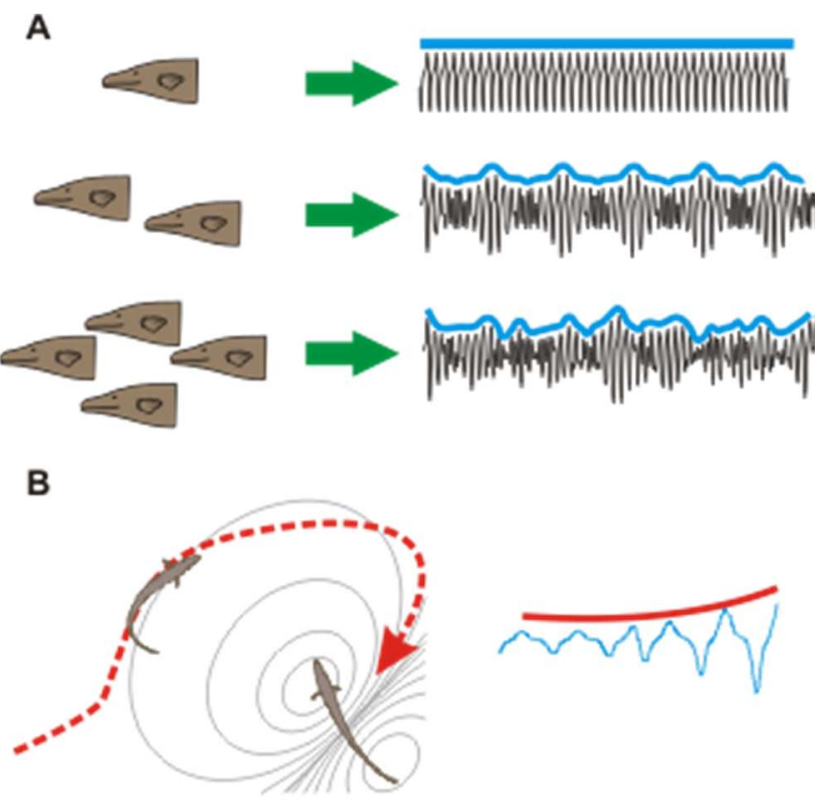

C

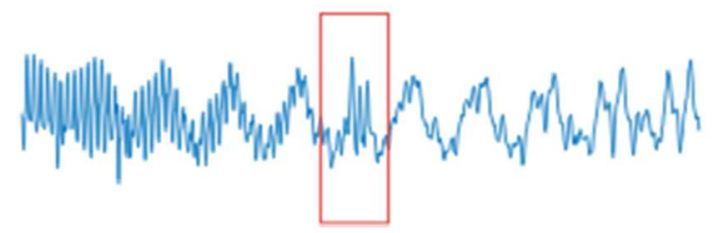

\section{Figure 1}

Noise in the electrical image arises from movement and conspecifics. A) Individual fish produce an extremely regular EOD (black), resulting in an electrical field with a constant overall amplitude (cyan). The presence of additional fish results in amplitude modulations of that field (beat, cyan). Several fish interacting results in highly complex signals, making identification of chirps more difficult. B) The strength of amplitude modulation contrast changes as the result of movement. As fish interact, the strength of the beat changes, resulting in very low frequency contrast changes (envelope, red). C) An example chirp (highlighted in the red box) occurring in the group condition consisting of a complex background beat AM (blue line). 
A

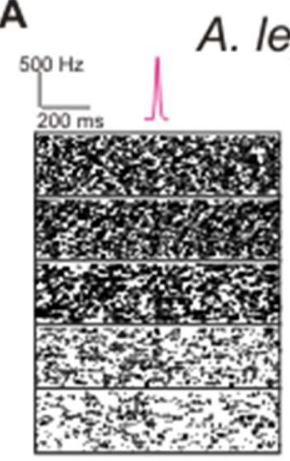

B
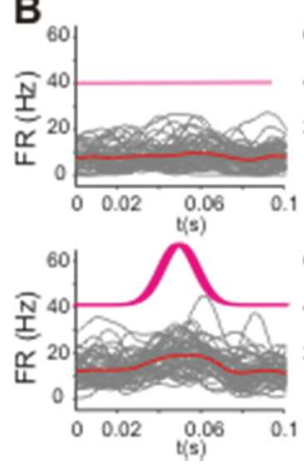

Figure 2
A. alb
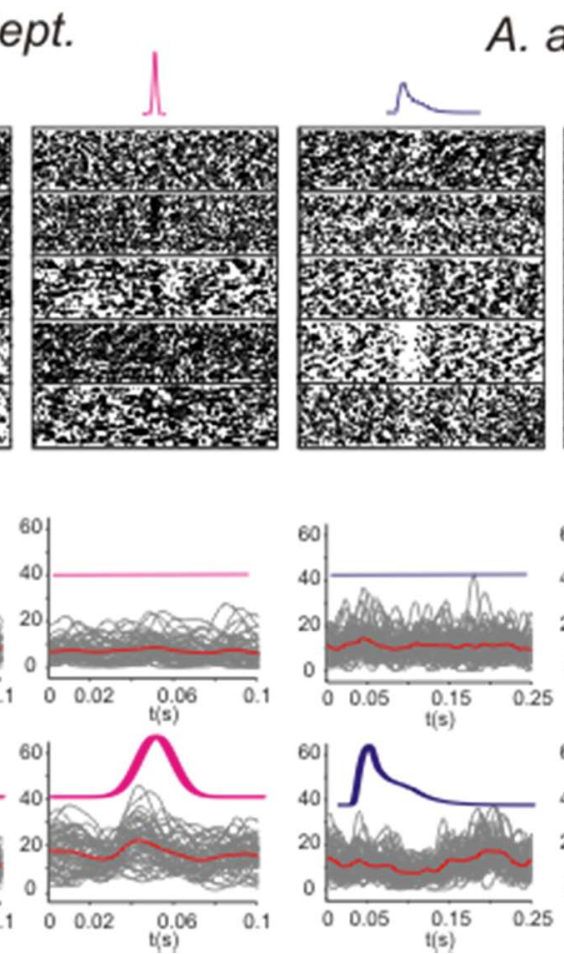
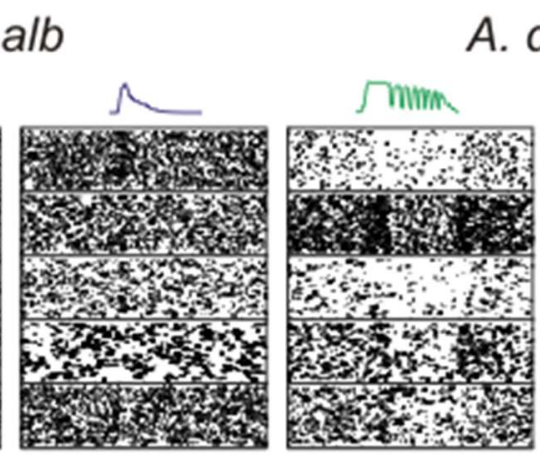

A. dev
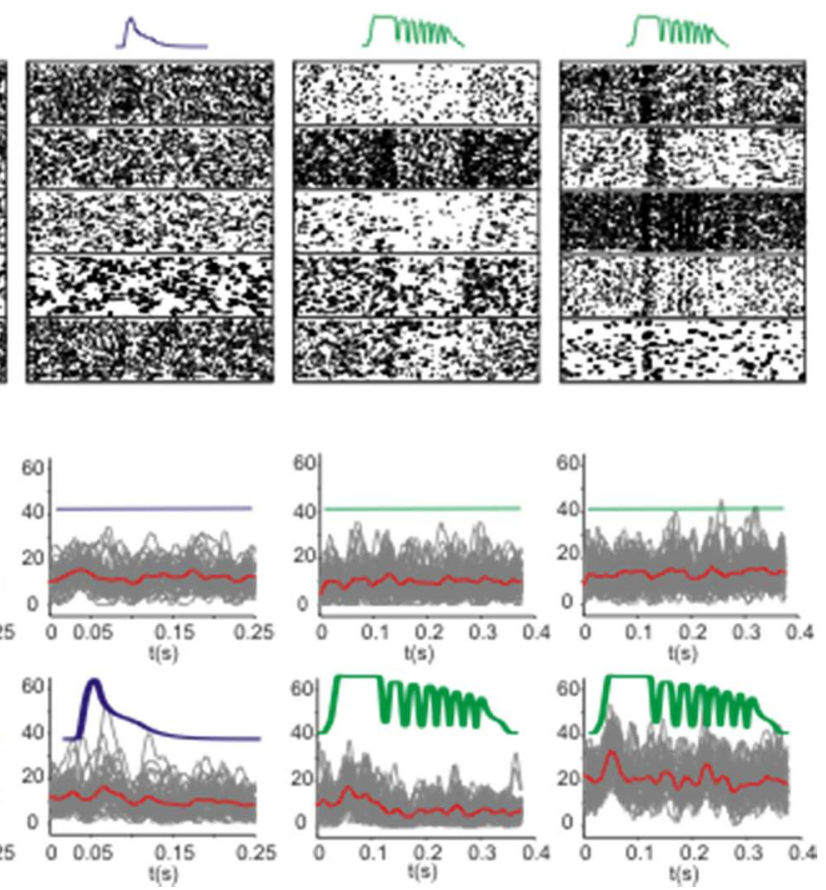

Responses to chirps on changing envelopes vary with beat. A) A selection of representative OFF-cells demonstrate that in A. leptorhynchus (magenta) chirps on all frequencies ( $10 \mathrm{~Hz}$ beat left, $100 \mathrm{~Hz}$, right) produce excitation, while in both $A$. albifrons (blue) and $A$. devenanzii (green) low frequencies produce inhibition that lasts the length of the chirp, and excitation on high frequency beats. Shown are responses to Chirp 4 from each species (See Table 1 for details). Chirp amplitudes were varied and randomized for beat phase and timing, thus response strength varies from one chirp repetition to the next. Each chirp was presented 76 times over the course of the 2 minute stimulus. B) Weighted firing rates drawn from randomized chirp repetitions. For each species beat responses (top) and chirp responses (bottom) are shown. Individual Gaussianconvolved firing rate traces (gray) are randomly selected from all pooled responses and multiplied by their KL divergence value, as was done in the detection analysis. These weighted individual chirp responses and beat responses were compared to determine error in chirp detection. The mean of these selected responses is shown in red. 
A
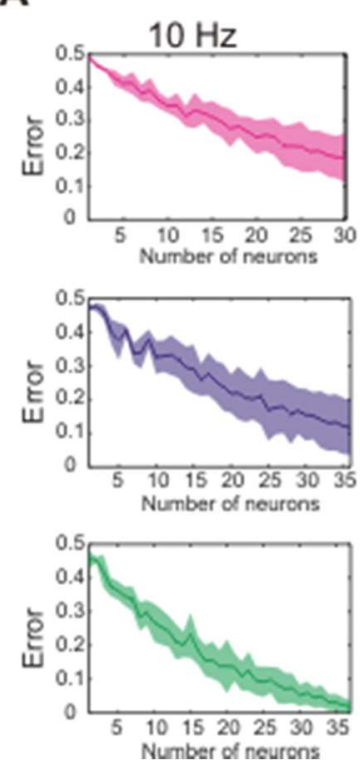

B
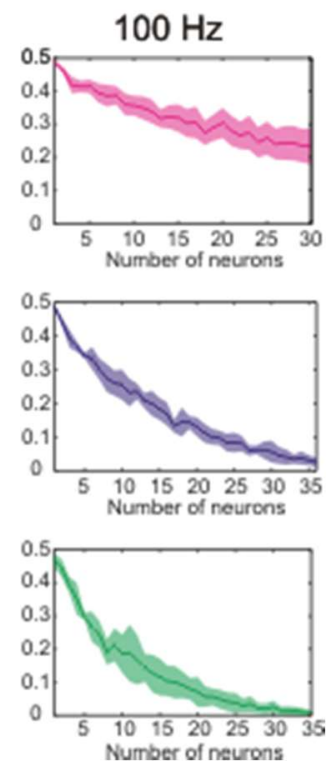

Figure 3

Detection accuracy and efficiency in a dyad condition varies across species. A) Mean detection error rate of all chirps show that $A$. devenanzii detect chirps most efficiently in both 10 and $100 \mathrm{~Hz}$ (B) beat conditions. Detection error probability as a factor of number of neurons included in the analysis is shown. Error probability is the probability that an ideal observer is able to correctly assign a spike train as a chirp or beat response. Mean rates for all four chirps are shown as a solid line, standard deviation is shaded (A. leptorhynchus: magenta, $A$. albifrons: blue, $A$. devenanzii: green). All three species indicate that detection is possible in both 10 and $100 \mathrm{~Hz}$ conditions, but that $A$. leptorhynchus would require more neurons than we sampled $(\mathrm{n}=30)$ to achieve $<.10$ error rate $(90 \%)$ accuracy. A alibifrons $(\mathrm{n}=35)$ is more efficient than $A$. leptorhynchus, but $A$. devenanzii $(\mathrm{n}=35)$ is the best at detection. 
A
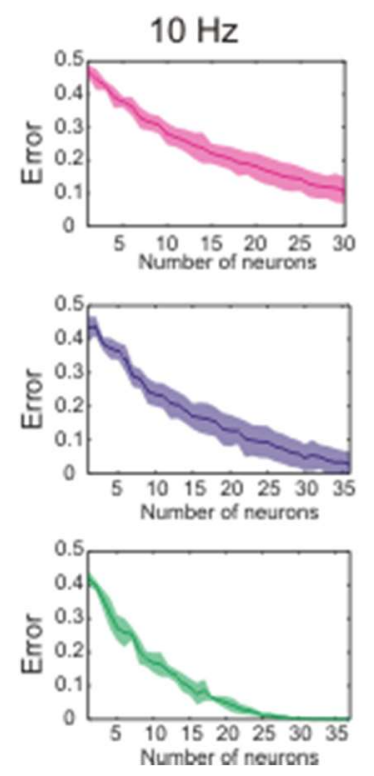

B
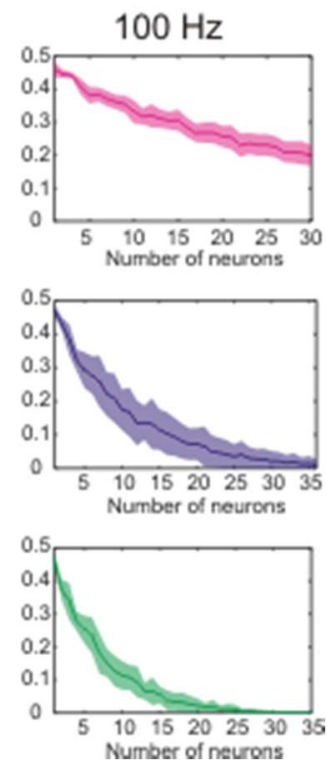

Figure 4

Discrimination accuracy in a dyad condition also varies by species. A. devenanzii neural responses allow for efficient discrimination between chirps. Like detection, all species could potentially discriminate chirps in all beat conditions (10Hz: A, $100 \mathrm{~Hz}$ : B), but $A$. devenanzii chirp responses result in very efficient discrimination between chirps reaching $<.10$ accuracy in under 15 neurons. Mean discrimination is shown in solid, standard deviation is shaded (A. leptorhynchus: magenta, $A$. albifrons: blue, $A$. devenanzii: green). 
A

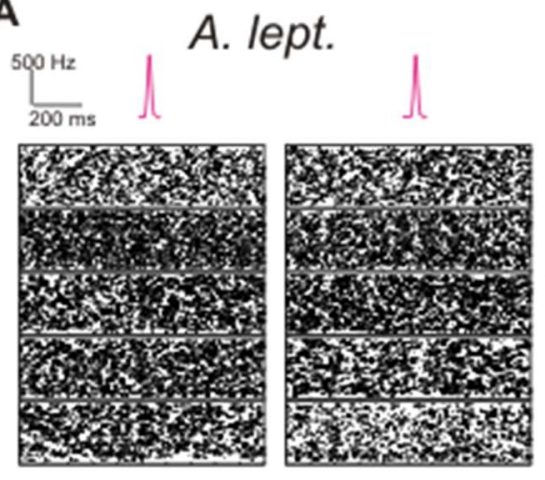

B
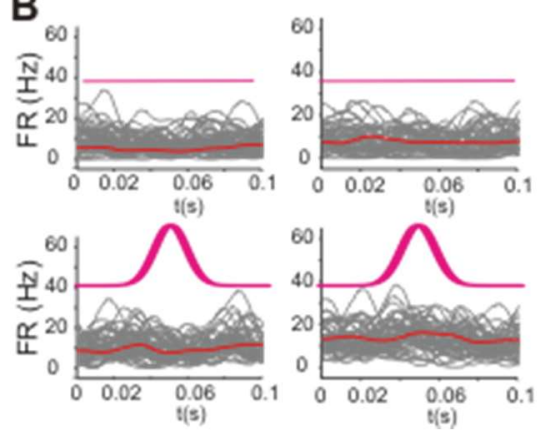

A. alb
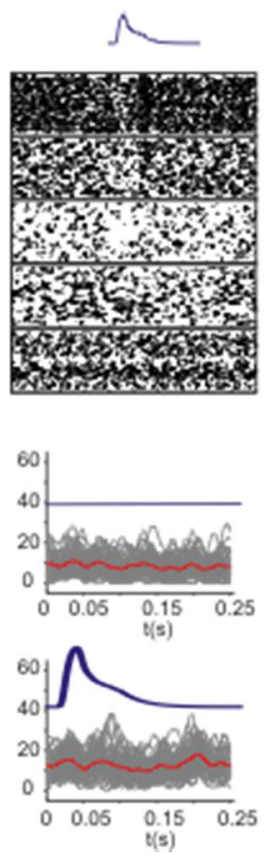
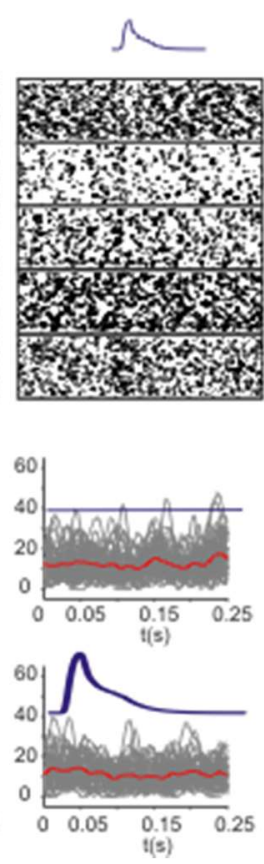

A. dev
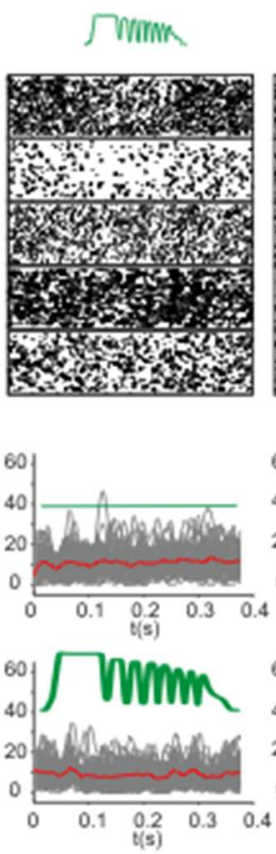
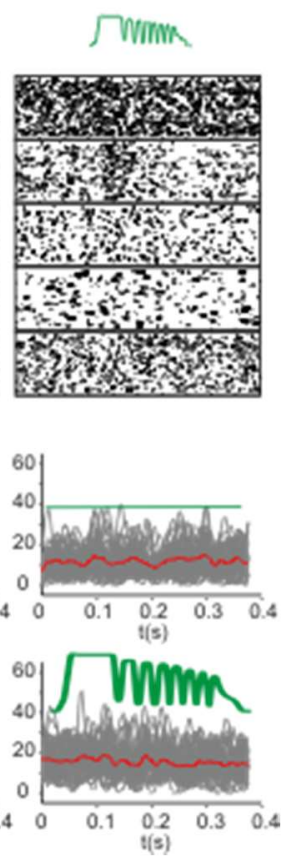

Figure 5

Responses to chirps in noisy conditions are subtle. A) A selection of representative OFF-cells showing chirp responses to chirps embedded in an envelope simulating group interactions. The chirps shown here are identical to those in Figure 2. Responses to these chirps appear to follow the same overall pattern as in the dyad condition, but the magnitude of the response is much subtler. (A. leptorhynchus: magenta, A. albifrons: blue, A. devenanzii: green) B) Weighted firing rates also indicate subtle chirp responses. Similar to Figure 2, randomly selected chirp responses are shown in gray, mean response averages are shown in red. These responses also indicate attenuated neural representation of chirps in noise. 
A
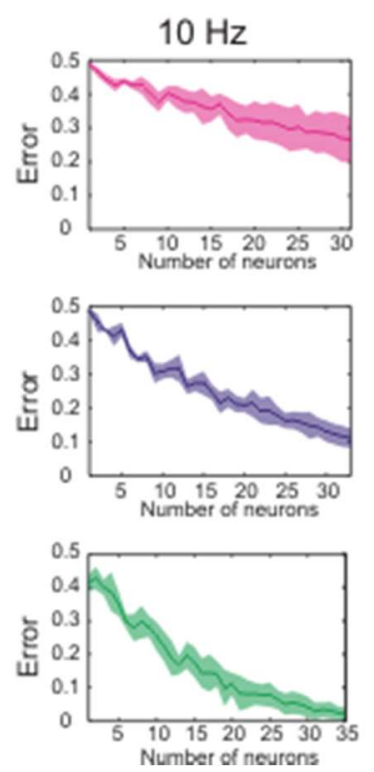

B
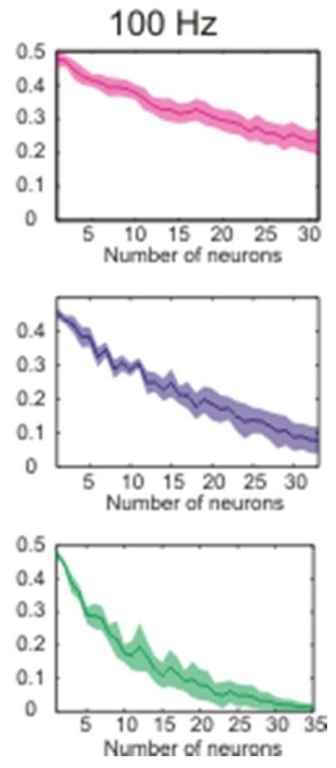

Figure 6

Detection accuracy and efficiency in a noise condition varies across species. A) Mean detection error rate of all chirps show that $A$. devenanzii detect chirps most efficiently in both 10 and $100 \mathrm{~Hz}$ (B) beat conditions. Mean rates for all four chirps are shown in as a solid line (A. leptorhynchus: magenta, A. albifrons: blue, $A$. devenanzii: green), standard deviation is shaded. A. devenanzii show markedly steeper decreases in detection errors. Even though the observed chirp responses are subtler than in the dyad condition our analyses suggest that they still provide enough information for accurate detection of chirp occurrence. 
A
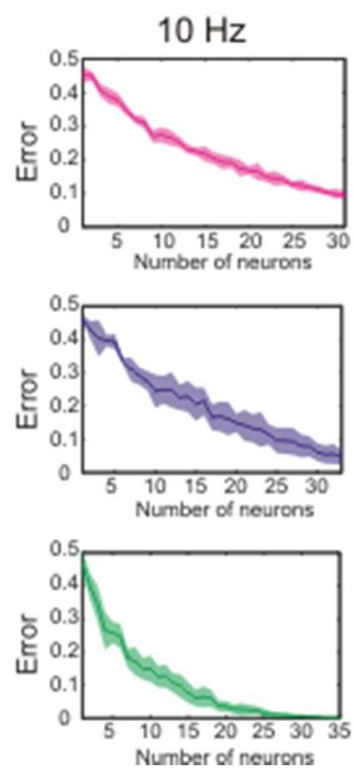

B
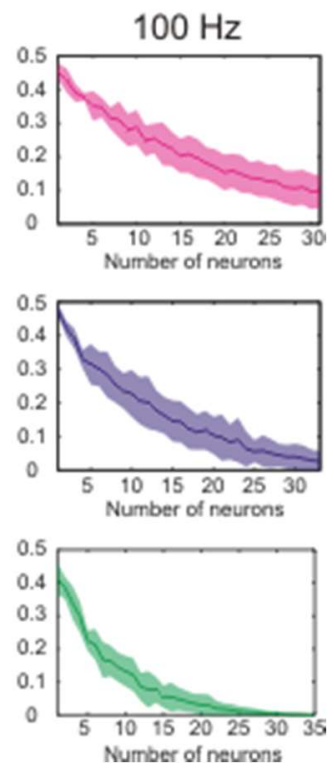

Figure 7

Discrimination accuracy in a noise condition also varies by species. A) $A$. devenanzii neural responses allow for efficient discrimination between chirps. Like detection, all species could potentially discriminate chirps in all beat conditions, but $A$. devenanzii chirps responses result in very efficient discrimination between chirps without much difference from the dyad condition. Mean discrimination is solid, standard deviation is shaded. (A. leptorhynchus: magenta, $A$. albifrons: blue, $A$. devenanzii: green) 


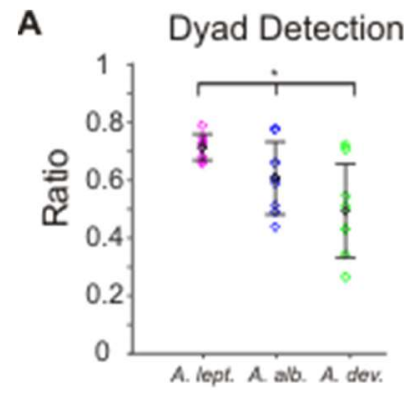

B

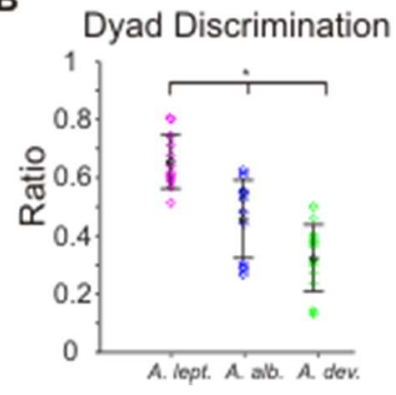

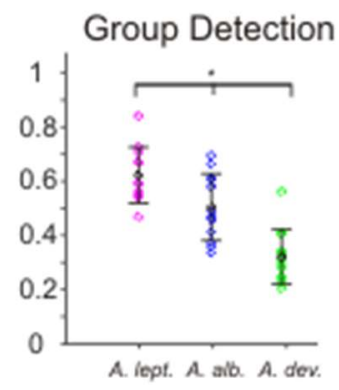

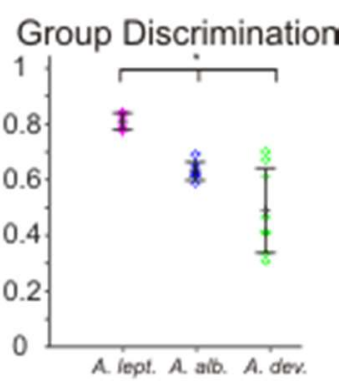

Figure 8

Detection and discrimination efficiency varies between species. A) Detection efficiency as a ratio between a sample of 1 and a sample of 10 . To create a normalized measure of coding accuracy and the amount of information provided by each neuron the error level at n10 was divided by the error level at $\mathrm{n} 1$ for each chirp. In both noise conditions, $A$. devenanzii show markedly steeper decreases in detection errors. Shown are individual error levels for each chirp pair measured as determined from the curves in Figures 3, 4, 6, and 7. Mean and standard deviation are shown in black $(A$. leptorhynchus: magenta, $A$. albifrons: blue, $A$. devenanzii: green). B) Discrimination ratios

confirm $A$. devenanzii discrimination efficiency. Discrimination ratios were determined similarly to detection ratios. Like detection, $A$. devenanzii exhibit the steepest decrease in error rate. Asterisk indicates $\mathrm{p}<.05$. 


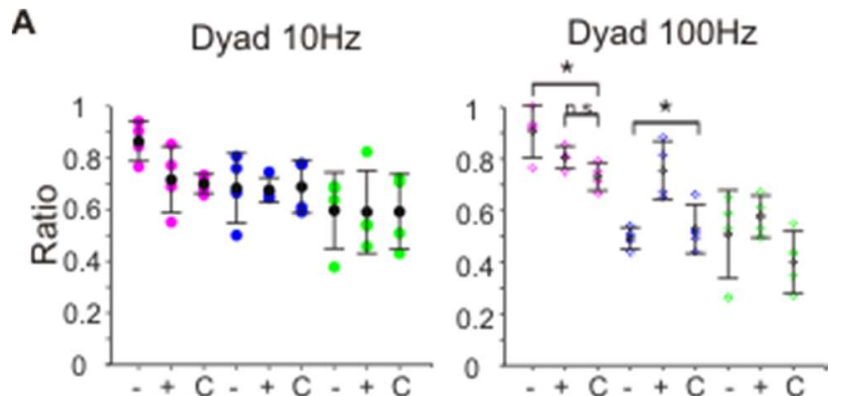

B

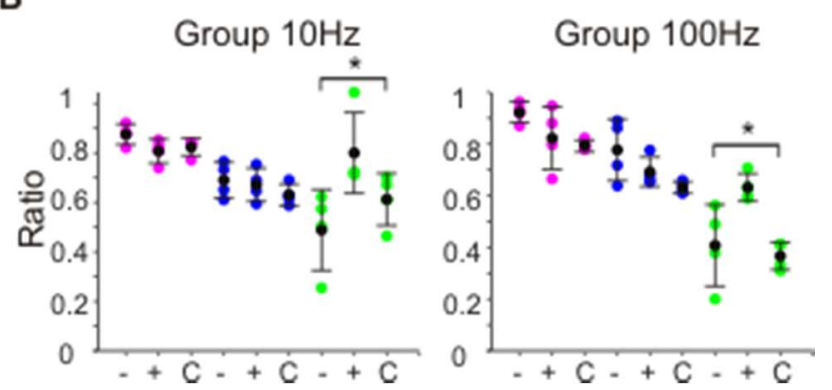

Figure 9

Coding accuracy varies between On- and Offcells in noise conditions. A) Detection ratios for dyad and noise conditions divided by ON$(+)$, OFF (-), and combined (C) analyses. While detection accuracy varies by species, $\mathrm{ON}$ - and OFF-cells perform equally as well in the dyad condition (A. leptorhynchus: magenta, A. albifrons: blue, A. devenanzii: green). B) However, in the noise condition, $A$. devenanzii shows coding is performed much more accurately by OFF-cells (ANOVA, $10 \mathrm{~Hz} p=0.0428,100 \mathrm{~Hz} p=.0101)$. Means and standard deviation are show in black. 
Figure 10

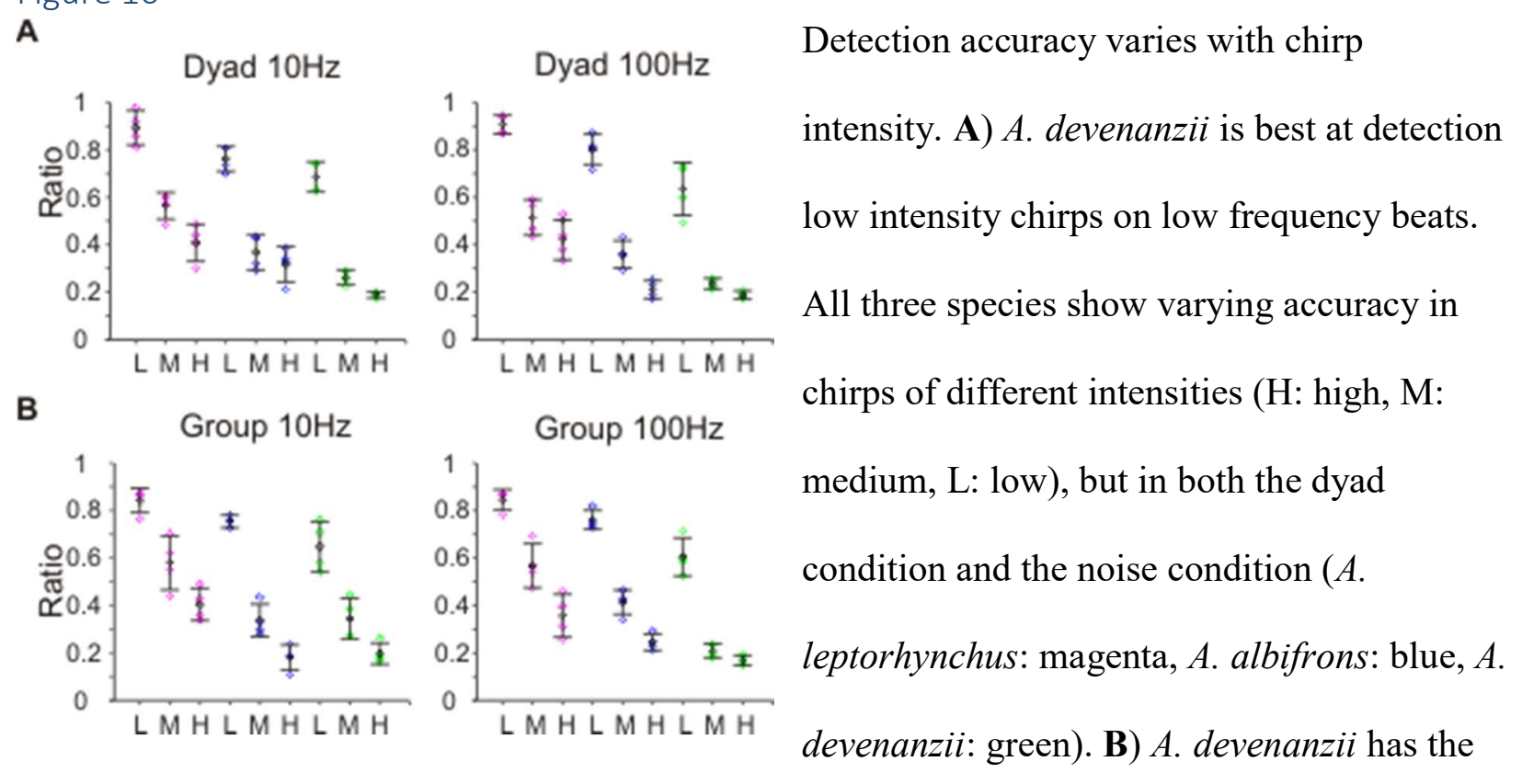

highest accuracy in detecting low intensity chirps. Means and standard deviation are shown in black. 
Figure 11

A

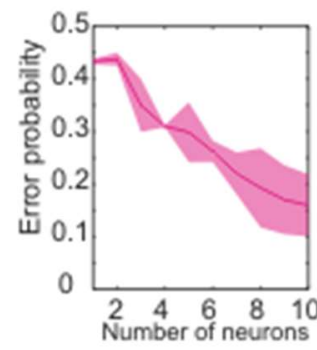

Mean Detection
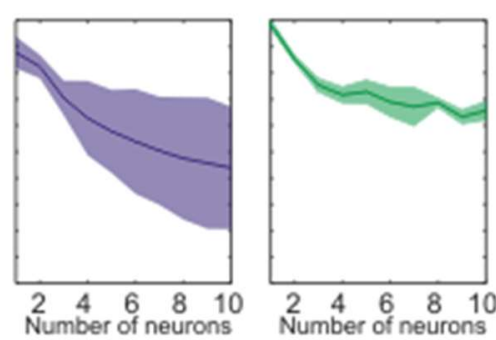

B

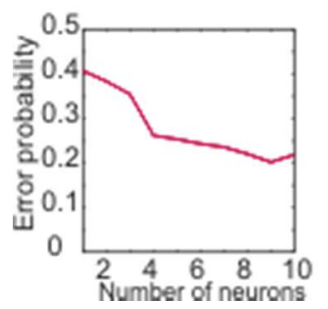

Mean Discrimination

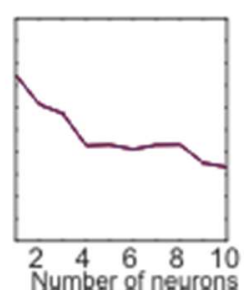

Response to A. leptorhynchus small chirps varies across species. A) We find that $A$. leptorhynchus (magenta) are best at detecting their own chirps in these conditions and $A$. devenanzii (green) perform worst. This suggest that while $A$. devenanzii chirp structure may be particularly conspicuous, there are also differences in chirp encoding, that make them adept at coding these chirps over heterospecific chirps. Shaded area is standard deviation B) Discrimination of $A$. leptorhynchus small chirps. A. devenanzii responses also produce poor discrimination of A. leptorhynchus chirps, while $A$. albifrons and A. leptorhynchus may be able to discriminate small chirps, it is still error prone. 


\section{Supplemental information}

S1

A
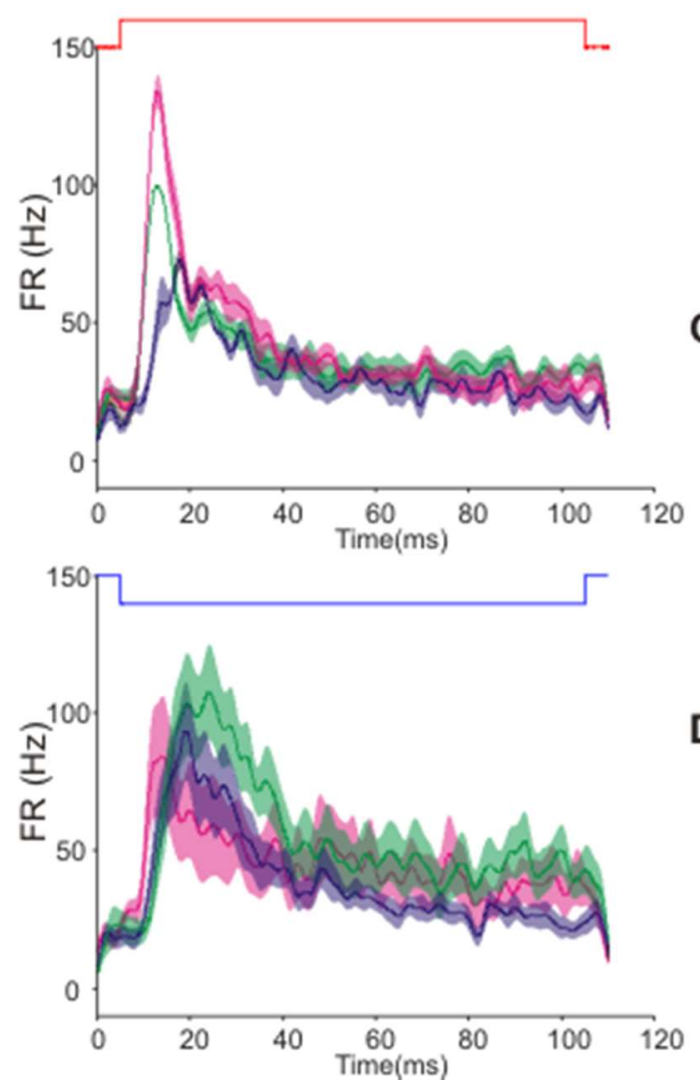

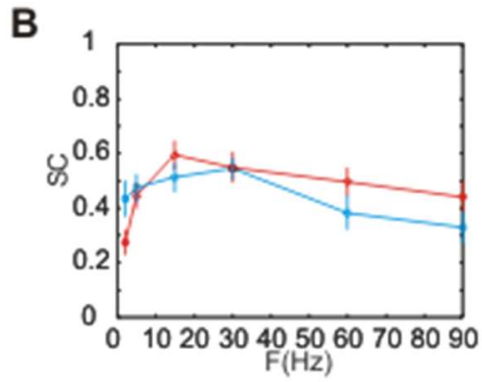

C
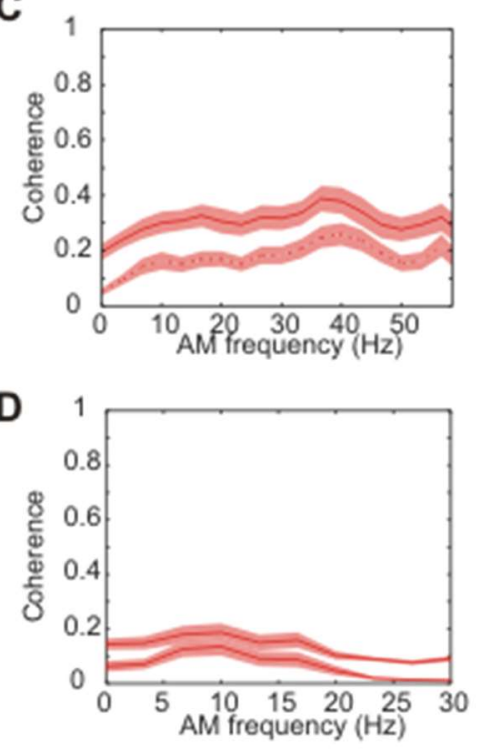
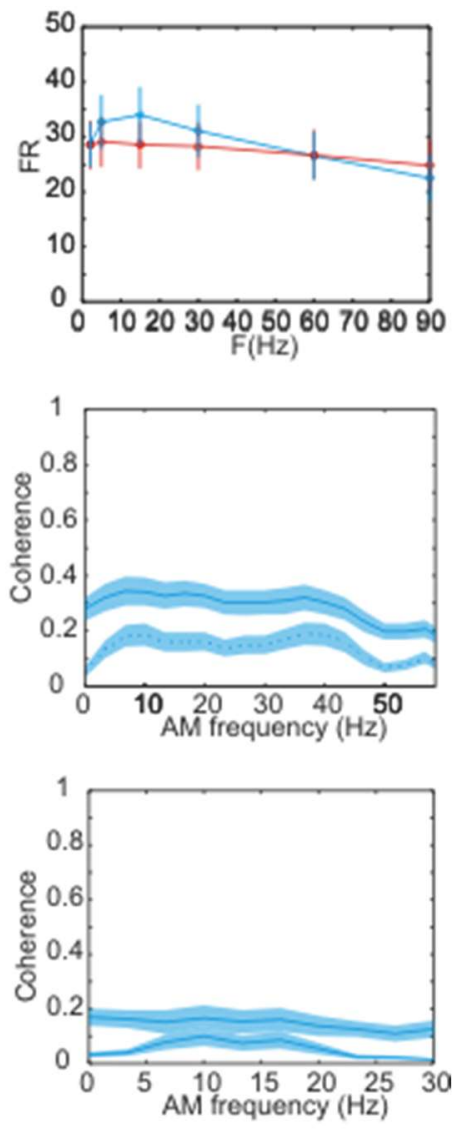

Response properties show differences across species. A) Adaptation to square pulse stimulation shows differences in firing properties Firing rate from ON- (top) and OFF-cells (bottom) to $100 \mathrm{~ms}$ long square pulse stimulation show that $A$. leptorhynchus (magenta) ON- cells are particularly bursty, possibly enabling better detection of extremely short chirps, but poor for coding duration. Comparatively, A. devenanzii (green) and A. albifrons (blue) are less prone to bursting. B) A. devenanzii show broad tuning to sine stimuli. Synchronization Coefficient (left) and firing rate (right) in response to sine waves of frequencies from $2-90 \mathrm{~Hz}$ (details about analysis can be found in previous chapters (Allen and Marsat, 2019) show that while ON-cells (red, standard deviation shown) are lower tuned than previously reported values for $A$. leptorhynchus, OFF-cells are higher tuned (cyan) (Krahe et al., 2008), suggesting less of a dichotomy in cell type and tuning. Both types are higher tuned than A. albifrons (Allen and Marsat, 2019). C) A. devenanzii coherence to $0-60 \mathrm{~Hz}$ white noise is broad and does not show large differences between ON- and OFF- cells. Like sinusoid responses, Previous data indicate that $A$. albifrons exhibit peak frequency sensitivity at low frequencies (Allen and Marsat, 2019), and A. leptorhynchus ON-cells (red) are higher tuned than OFF-cells (cyan) (Krahe and Chacron, 2008). A. devenanzii show relatively broad tuning in both cell types. Upper bound coherence 
indicated by solid line, lower bound dashed, standard deviation shaded. D) A. devenanzii envelope coding is surprisingly poor. $A$. devenanzii responses to low frequency envelope coding shows very little envelope coding. This suggests that they are using chirp features other than envelope for detecting chirp occurrence. Upper bound coherence indicated by solid line, lower bound dashed, standard deviation shaded. 


\section{Chapter 5: Discussion}

\section{Summary of the data}

With this dissertation, I have shown the diversity of chirp coding in three species of apteronotid fish and demonstrated several important principles of neural coding and signal perception. Specifically, I have shown that the context in which a signal is received alters the coding strategy used to represent that signal (Chapters 2 and 3) and that the presence of noise may actually improve the perception of communication in species that are well adapted towards life in groups (Chapter 4). I have also provided evidence of sender-receiver matching in the neural code, showing that the coding strategies adopted by each species is particularly tailored to their own chirp structures (Chapters 3 and 4).

\section{Chirp coding strategies vary across species}

Chapter 2 of this dissertation demonstrates that in A. leptorhynchus two distinct coding strategies are used for different perceptual tasks. One coding strategy, stereotyped bursting, allows for efficient signal detection, but does not enable discrimination between chirps. The other coding strategy, increases in tonic firing rate, permits chirp discrimination. Further, it is not the structure of the chirp that mediates the switch in firing pattern that is evoked by chirps, but rather the social context in which the chirp is presented. A. leptorhynchus small chirps span less than a full cycle of the beat. Consequently, the same chirp will appear as a sharp decrease in amplitude, a sharp increase, or a mix of the two, depending on the phase of the beat at which the chirp starts (Benda et al., 2005). It may be due to this aspect of chirp structure that the sensory system of $A$. leptorhynchus uses bursts to enhance detectability rather that optimizing the coding of these chirps' properties. At higher beat frequencies both big and small chirps span more than 
one beat cycle, reducing the effect of phase on the electrical image the fish receives. This change in the signal structure allows the system to encode details about both chirp duration and frequency. The variability of the response to chirps on high frequency beats was described first for big chirps (Marsat and Maler, 2010) and confirmed for small chirps (Metzen and Chacron, 2017). These graded, non-redundant responses, proportional to chirp features, contain enough information that chirps with subtly different properties may be discriminated from one another.

We confirmed our hypothesis about the discriminability of chirps in relation to beat frequency by assessing the performance of the fish with a habituation-dishabituation assay. The behavioral response of the fish shows that these two codes mediate different perceptual tasks. The fish tested were able to perceive and react to slight differences in chirps that are encoded via graded, heterogeneous firing rates. Chirp stimuli that elicited stereotyped bursts, however, did not result in discrimination behavior.

In Chapter 3 the association between beat frequency and chirp coding is further investigated. The effect of beat frequency on chirp encoding is opposite in A. albifrons as in A. leptorhynchus. ELL neurons in A. albifrons are able to detect chirp occurrence and do encode chirp identities on low frequency beats. However, they discriminate between chirps less accurately when chirps are presented on high frequency beats. Rather than producing discrete burst responses for small chirps and tonic responses for big chirps, all chirps in this species are encoded via graded and heterogeneous increases or decreases in firing rate. This difference in coding strategy is the result of both differences in signal structure and ELL physiology. Because chirps in this species are much longer than those of A. leptorhynchus, even chirps on low frequency beats are not affected by beat phase and chirp timing in the same way as $A$. leptorhynchus and thus signal structure does not preclude discrimination. Due to this difference, 
we suggest that this species may not require the same invariant coding strategy for some signals as A. leptorhynchus.

We also asked whether chirping behavior could explain differences in chirp coding efficiency for high- and low-frequency beats. We found that the pattern of chirp use during common social interactions is very different from that of closely related species: A. albifrons produces very few chirps and do not respond to each other with echo chirps. Infrequent chirping is particularly pronounced in the context of high-frequency beats suggesting that certain types of interactions are not mediated by chirps as in A. leptorhynchus. We suggest that the observed differences in chirp coding accuracy on high frequency beats may reflect a relaxation in demands for exchanging information via chirps in this context.

The data in Chapter 4 show that $A$. devenanzii coding is particularly efficient at detecting and discriminating chirps in all contexts. The response patterns present notable differences compared to other species that can be related to their high coding efficiency for all signals. Chirps presented on low frequencies generally produce inhibition lasting the duration of the chirp, much like what is observed in A. albifrons responses, where coding accuracy is high. On high frequency beats, chirps evoke an increase in firing that that is also proportional to the length of the chirp, more similar to what is observed in A. leptorhynchus. What results is a neural code that enables accurate discrimination of chirps in all social contexts. Unique to this species is the multiple peaks in firing rate, corresponding to the multiple frequency peaks contained within the complex chirps that males in this species produce (Zhou and Smith, 2006). Therefore, high coding accuracy is paired with a chirp repertoire that varies significantly in structure. This coding scheme may be reflective of this species' ethology, as they are generally more social than A. leptorhynchus and A. albifrons. Additionally, EOD frequency is not sexually dimorphic in this 
species (Zhou and Smith, 2006). This means that chirps of all types, even the multi-peaked chirps produced mainly by male fish are more likely to be encountered on a wide variety of beat frequencies, reducing the need for beat-context specific feature extraction and increasing the need for chirp assessment in all circumstances.

\section{Evidence for sender-receiver matching}

In this dissertation I show that not only are coding strategies different between these species, but also that these differences can be understood in the light of sender-receiver matching principles. Chirp coding and the ethology of chirp use are well-studied in A. leptorhynchus. However, it is impossible to draw conclusions about the ubiquity or uniqueness of a given coding strategy or adaptation is it is not compared across several species, each facing their own sensory challenges and behavioral needs. Ideally, a large number of species should be compared to truly assign evolutionary adaptation as the cause of the observed differences, but looking at a few species as we did here is a step in that direction and is sufficient to identify key neural differences that relate to behavioral and signal differences.

In chapter 3, the differences between A. albifrons and A. leptorhynchus neurophysiology are compared extensively. These data allow us to demonstrate that specific differences in ELL neuron response properties can be adaptive for coding unique aspects of conspecific chirps. Our results confirm that pyramidal cells in the LS of $A$. albifrons are more low-pass than those of $A$. leptorhynchus. This finding parallels the low frequency envelopes produced by long A. albifrons chirps. However, low frequency tuning to AM is not automatically helpful. We found that the coding of low-frequency envelopes was relatively poor, particularly in OFF cells, which -in $A$. leptorhynchus- are best at coding chirp envelopes. However, if envelope response is synthesized in the electroreceptors, before the pyramidal cells, low frequency tuning to AM could still assist 
with coding chirps as input to the pyramidal cells would contain those low-frequencies (Metzen et al., 2018).

In Chapter 3, the role of burst coding in A. albifrons is also explored. As suggested above, burst coding of chirps may be unique to A. leptorhynchus due to the extremely short duration of their chirps compared to other apteronotids. We explored the propensity for burst firing in A. albifrons and found that while this species is capable of coding stimulus features via bursts, chirp stimuli, even those known to evoke bursts in A. leptorhynchus, do not cause bursts in the majority of $A$. albifrons neurons. This suggests that not only are differences in signal structure driving coding differences, but that there are also underlying changes to ELL physiology.

In Chapter 4, adaptations for social lifestyle are investigated. In apteronotids, group size varies between species and the presence of multiple fish in a small area creates a complex electrical environment (Stamper et al., 2010), increasing the difficulty of detecting chirps and encoding chirp details. Three species of weakly electric fish with varying levels of gregariousness were compared to determine how well chirps encoded in these complex naturalistic scenes. I found that while the addition of social noise did little to hamper coding as compared to a less noisy but realistic condition. While these data are unexpected from my predictions, in other systems, noise may improve signal detection (Hänggi, 2002; Wells et al., 2005; Zeng et al., 2000) and responses to communication signals may be even more salient against a time-averaged background of noise

What is most noteworthy about my findings in Chapter 4 is that in all conditions, both low and high noise, coding accuracy did vary systematically across the three species. Supporting my hypothesis that social context influences the adaptations of a sender-receiver system, the 
most social species was indeed best at both detecting and discriminating among chirps. My results indicate that coding is a product of changes in neural properties such as frequency tuning, changes in coding strategy used, as well as differences in chirps that are adapted to be long (Zhou and Smith, 2006), conspicuous (Petzold et al., 2016), and information rich in the more social species. It was surprising to find that $A$. leptorhynchus exhibited the poorest accuracy in chirp detection, rather than the more aggressive and solitary A. albifrons. It could be largely due to species differences in chirps' structure despite adaptation for the efficient encoding of conspecific chirps. A. leptorhynchus performs well at encoding chirps in the simplest conditions (Chapter 2 and 3) and we showed that its nervous system has specific mechanism to do so. As a result, this species encodes its own chirps better than the other species encode A. leptorhynchus chirps. The more challenging signals used in Chapter 4, both with and without social noise, result in A. leptorhynchus chirps being poorly discriminated by conspecifics. Note that $A$. leptorhynchus chirps are less conspicuous than chirps of the other two species (Petzold et al., 2016). Furthermore, phylogenetic analysis indicate that short chirp is the basal condition in apteronotids (Smith et al., 2016), thus the longer chirps of A. albifrons an A. devenanzii could be an adaptation to make chirps more conspicuous. It could be predicted that, if chirp detectability in difficult conditions is not efficient enough in A. leptorhynchus, evolutionary pressures might further enhance coding mechanisms or shape the conspicuousness of the chirps in this species.

\section{Future Directions}

\section{Behavioral analysis of perception}

I have shown in my work, that there are a number of firing patterns that are evoked by different types of chirps, and that these firing patterns vary between species. From my 
physiological data, there is evidence that some of these chirp-evoked firing patterns do not contain enough information for the animal to accurately discriminate between chirps (Allen and Marsat, 2018; Allen and Marsat, 2019). Particularly, the small chirps of A. leptorhynchus, although we also see evidence that certain A. albifrons chirps are also indistinguishable. However, a different experiment analyzed with a less conservative measure suggests that in some conditions, these chirps may actually be discriminable (Chapter 4). In this experiments we used chirps at opposite ends of the natural range and the complex influence of phase on signal structure is thereby minimized. We can then question whether these signals are indeed categorically perceived or if there is some perception of difference. Responses of downstream targets of ELL cells do not provide a clear answer. For some cells of the TS, there is evidence that at least at higher levels of signal processing, A. leptorhynchus small chirps are indeed indistinguishable from each other (Aumentado-Armstrong et al., 2015). Other TS neurons respond with a more dense descriptive code (Vonderschen and Chacron, 2011). A more complete analysis of responses at different level to chirp stimuli finely covering the natural repertoire could provide some answers to these questions. However, with physiological data, we are restricted to speculating about what the animal may be perceiving or what information is used to evoke behavior. To confirm our hypotheses, it is important to include behavioral data showing that the animal is or is not sensitive to aspects of the stimuli as we predict. In Chapter 2, I provide this evidence using a habituation-dishabituation assay to assess how well $A$. leptorhynchus is able to discriminate between chirps in different contexts, and show that small chirps do not elicit discrimination behaviors on low frequency beats. This suggests that even if there is information permitting small chirp discrimination in the ELL, it is either lost at higher 
sensory stages or irrelevant enough to the animal that it does not contribute to the subsequent behavior.

Currently, there are no comparable studies published on the other two species included in this dissertation. Due to inconsistencies in analyses in determining whether chirps are distinguishable, particularly for A. albifrons, it is important to follow the physiologic data presented here with similar behavioral tests. While a very conservative metric (van Rossum, 2001) suggests that in A. albifrons some chirps are indistinguishable on high frequency beats, a more liberal metric suggests that there may be enough information represented in the ELL to accomplish a discrimination task. Relatedly, it is worth considering the possibility that while the information about the chirp is present, it may not be significant enough to the fish to actually produce a behavioral response. This may be especially true of $A$. albifrons, a solitary and territorial species that chirps very infrequently (Allen and Marsat, 2019; Kolodziejski et al., 2007). Conversely, A. devenanzii is highly social, and chirps more frequently. These chirps are also complex and varied (Zhou and Smith, 2006) allowing these variations in chirps structure to carry information. It would be intuitive to say then, that there is more ethological motivation for the more social $A$. devenanzii to encode chirps more accurately and be better able to discriminate amongst them. My physiological data suggest that this is the case, by both conservative and liberal analyses, but it is imperative that these studies be followed by behavioral experiments to support the conclusions regarding perception of chirps drawn from these data.

\section{Modulation of chirp reception}

Working with wild caught animals has a number of advantages, in that we know that our animals have been raised in a natural environment, being exposed to realistic stimuli and express true wild-type behavior. We therefore expect that the effects we observe are representative of a 
general sensory state in these animals. However, there are many factors that could influence the perception of chirps in both short- and long-term contexts. Neuromodulation can change cell response properties for different behavioral states (Harris-Warrick and Marder, 1991). Hunger (Katz and Frost, 1996), social status (Simpson and Stevenson, 2015), and sex hormones (Remage-Healey et al., 2010) are all associated with distinct effects on sensory systems. While my work has characterized baseline chirp coding properties, the effects of neuromodulation on chirp reception are largely unknown.

Breeding condition is a potentially critical source of neuromodulation of chirp reception. For each chapter, adult animals of both sexes were used for all experiments, however these animals are difficult to coax into breeding condition in captivity. Chirping serves an important role in courtship rituals (Hagedorn and Heiligenberg, 1985; Henninger et al., 2018) and entering breeding condition can affect the quality and quantity of chirps produced (Smith, 2013). It is thus likely that this behavioral state also affects the reception and encoding of chirps. Previous work in A. leptorhynchus shows that bath application of serotonin enhances pyramidal cell excitability and responsiveness to small chirps on low frequency beats (Deemyad et al., 2013) suggesting a mechanism for altering sensitivity to chirps and possibly even coding accuracy in response to behavioral need. There are a multitude of other sources of modulation that could arise as a result of entering into a breeding state that may also increase sensitivity to chirp signals (Marquez et al., 2013). Certainly, this work should be coupled with more behavioral studies into the natural breeding behavior of $A$. albifrons and $A$. devenanzii both in lab settings and the field to better understand the circumstances of chirp usage in these species. 
The work in this dissertation concludes that there are physiologic differences in LS neurons between $A$. leptorhynchus, A. albifrons, and A. devenanzii as demonstrated by the differences in neural response properties. I have characterized some of these properties, such as proportion of burst to tonic firing, frequency tuning, and adaptation rates. What remains unaddressed are the underlying mechanisms driving these differences. While the gross anatomy, and presumably the gross circuity of these species' brains appears to be well-conserved, any number of differences in microcircuitry, transmitter expression, receptor distribution, ion channels or other membrane proteins could give rise to large differences in response properties. Small differences in receptor expression can lead to large changes in social behavior among closely related species, as famously demonstrated in voles (Insel and Shapiro, 1992) and receptor expression in areas of sensory processing such as the olfactory bulb are vital for promoting social behavior and recognition of conspecific signals (Broad et al., 2006). The prevalence of receptor expression as a mechanism for mediating social behavior and processing of social communication suggest a promising point to begin investigation into the mechanisms driving the sender-receiver matching I have observed.

\section{Conclusions}

Communication is an important driver of sensory system evolution. For organisms to exchange information with each other, each signal must be detectable from the background noise of the sensory environment, as well as discriminable from other signals with different meanings. To achieve these tasks of detection and discrimination, organisms must adapt both the structure of the communication signal, as well as match the sensitivity of their sensory systems to match signal properties. Further, sensory coding strategies that enable these tasks must also be adapted to the animal's life history and behavioral patterns. By comparing how sensory coding is 
achieved across several species, we can draw conclusions about which strategies may be generalizable to all organisms facing a given sensory task, and which strategies are uniquely adapted to an individual species' needs. 


\section{Works Cited}

Allen, K. M. and Marsat, G. (2018). Task-specific sensory coding strategies matched to detection and discrimination behaviors in Apteronotus leptorhynchus. J. Exp. Biol. 221,.

Allen, K. M. and Marsat, G. (2019). Neural processing of communication signals: The extent of senderreceiver matching varies across species of Apteronotus. eneuro ENEURO.0392-18.2019.

Aumentado-Armstrong, T., Metzen, M. G., Sproule, M. K. J. and Chacron, M. J. (2015). Electrosensory Midbrain Neurons Display Feature Invariant Responses to Natural Communication Stimuli. PLoS Comput. Biol. 11, 1-28.

Benda, J., Longtin, A. and Maler, L. (2005). Spike-frequency adaptation separates transient communication signals from background oscillations. J. Neurosci. 25, 2312-21.

Broad, K. D., Curley, J. P. and Keverne, E. B. (2006). Mother-infant bonding and the evolution of mammalian social relationships. Philos. Trans. R. Soc. B Biol. Sci. 361, 2199-2214.

Deemyad, T., Metzen, M. G., Pan, Y. and Chacron, M. J. (2013). Serotonin selectively enhances perception and sensory neural responses to stimuli generated by same-sex conspecifics. Proc. Natl. Acad. Sci. U. S. A. 110, 19609-14.

Hagedorn, M. and Heiligenberg, W. (1985). Court and spark: electric signals in the courtship and mating of gymnotoid fish. Anim. Behav. 33, 254-265.

Hänggi, P. (2002). Stochastic resonance in biology how noise can enhance detection of weak signals and help improve biological information processing. ChemPhysChem 3, 285-290.

Harris-Warrick, R. M. and Marder, E. (1991). Modulation of neural networks for behavior. Annu. Rev. Neurosci. 14, 39-57.

Henninger, J., Krahe, R., Kirschbaum, F., Grewe, J. and Benda, J. (2018). Statistics of natural communication signals observed in the wild identify important yet neglected stimulus regimes in weakly electric fish. J. Neurosci. 0350-18.

Insel, T. R. and Shapiro, L. E. (1992). Oxytocin receptor distribution reflects social organization in monogamous and polygamous voles. Proc. Natl. Acad. Sci. 89, 5981-5985.

Katz, P. S. and Frost, W. N. (1996). Intrinsic neuromodulation: altering neuronal circuits from within. Trends Neurosci. 19, 54-61.

Kolodziejski, J., Sanford, S. E. and Smith, G. T. (2007). Stimulus frequency differentially affects chirping in two species of weakly electric fish: implications for the evolution of signal structure and function. J. Exp. Biol. 210, 2501-2509.

Marquez, B. T., Krahe, R. and Chacron, M. J. (2013). Neuromodulation of early electrosensory processing in gymnotiform weakly electric fish. J. Exp. Biol. 216, 2442-2450.

Marsat, G. and Maler, L. (2010). Neural heterogeneity and efficient population codes for communication signals. J. Neurophysiol. 104, 2543-55.

Metzen, M. G. and Chacron, M. J. (2017). Stimulus background influences phase invariant coding by correlated neural activity. Elife 6,.

Metzen, M. G., Huang, C. G. and Chacron, M. J. (2018). Descending pathways generate perception of 
and neural responses to weak sensory input. PLOS Biol. 16, e2005239.

Petzold, J. M., Marsat, G. and Smith, G. T. (2016). Co-adaptation of electric organ discharges and chirps in South American ghost knifefishes (Apteronotidae). J. Physiol.

Remage-Healey, L., Coleman, M. J., Oyama, R. K. and Schlinger, B. A. (2010). Brain estrogens rapidly strengthen auditory encoding and guide song preference in a songbird. Proc. Natl. Acad. Sci. 107, 3852-3857.

Simpson, S. J. and Stevenson, P. A. (2015). Neuromodulation of social behavior. oxford Handb. Mol. Psychol. 27.

Smith, G. T. (2013). Evolution and hormonal regulation of sex differences in the electrocommunication behavior of ghost knifefishes (Apteronotidae). J. Exp. Biol. 216, 2421 LP-2433.

Smith, A. R., Proffitt, M. R., Ho, W. W., Mullaney, C. B., Maldonado-Ocampo, J. A., Lovejoy, N. R., Alves-Gomes, J. A. and Smith, G. T. (2016). Evolution of electric communication signals in the South American ghost knifefishes (Gymnotiformes: Apteronotidae): A phylogenetic comparative study using a sequence-based phylogeny. J. Physiol. 110, 302-313.

Stamper, S. A., Carrera-G, E., Tan, E. W., Fugère, V., Krahe, R. and Fortune, E. S. (2010). Species differences in group size and electrosensory interference in weakly electric fishes: Implications for electrosensory processing. Behav. Brain Res. 207, 368-376.

van Rossum, M. C. (2001). A novel spike distance. Neural Comput. 13, 751-763.

Vonderschen, K. and Chacron, M. J. (2011). Sparse and dense coding of natural stimuli by distinct midbrain neuron subpopulations in weakly electric fish. J. Neurophysiol. 106, 3102-18.

Wells, C., Ward, L. M., Chua, R. and Inglis, J. T. (2005). Touch Noise Increases Vibrotactile Sensitivity in Old and Young. Psychol. Sci. 16, 313-320.

Zeng, F.-G., Fu, Q.-J. and Morse, R. (2000). Human hearing enhanced by noise11Published on the World Wide Web on 23 May 2000. Brain Res. 869, 251-255.

Zhou, M. and Smith, G. T. (2006). Structure and sexual dimorphism of the electrocommunication signals of the weakly electric fish, Adontosternarchus devenanzii. J. Exp. Biol. 209, 4809-4818. 